R290

no. $26-442$

\author{
UNITED STATES \\ DEPARTMENT OF THE INTERIOR \\ GEOLOGICAL SURVEY \\ WATER RESOURCES DIVISION
}

\author{
FLOOD-PLAIN DELINEATION \\ For \\ ACCOTINK CREEK BASIN \\ FAIRFAX COUNTY, VIRGINIA
}

Open-File Report 76-442

Prepared in cooperation with the

County of Fairfax 
U.S. DEPARTMENT OF THE INTERIOR, GEOLOGICAL SURVEY, WATER RESOURCES DIVISIOI

\section{ABSTRACT-INDEX SHEET}

1. Title (all caps) FLOOD-PLAIN DELINEATION FOR ACCOTINK CREEK BASIN FAIRFAX COUNTY, VIRGINIA

2. Authors Pat L. Soule

8. Type of publication Open-File Report

9. Descriptors Flood Profile, Flood-plain delineation, Flood routing, Flood forecasting, Floodways, Design flood, Flood Control, Flood protection

10. Identifiers Flood-plain mapping, Flood zone, Fairfax County, Virginia

11. Abstract

Water-surface profiles of the 25-year and 100-year floods and maps on which the 25-, 50-, and 100-year flood limits are delineated for streams in the Accotink Creek basin are presented in this report. Excluded are segments of Accotink Creek within the Fort Belvoir Military Reservation. The techniques used in the computation of the flood profiles and delineation of flood limits are presented, and specific hydraulic problems encountered within the study area are also included. 

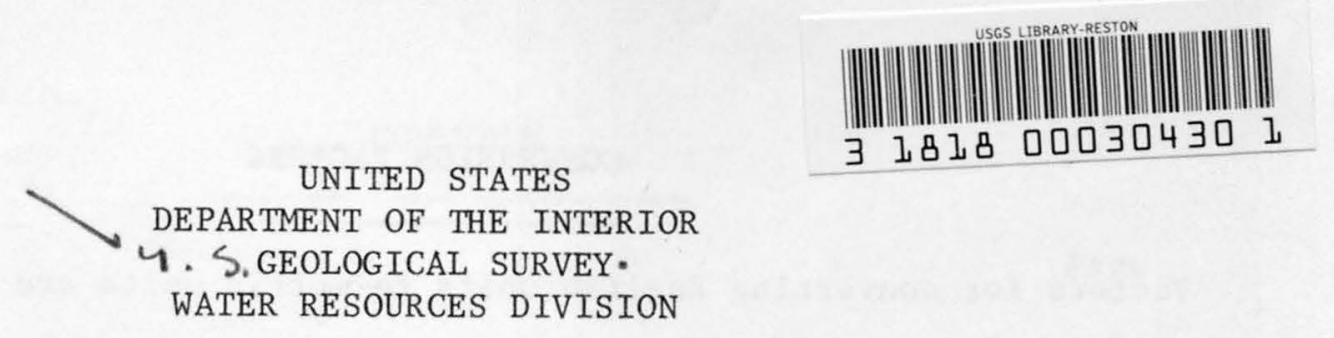

$$
\begin{gathered}
\text { [Reports-Open file } \\
\text { series }
\end{gathered}
$$

FLOOD-PLAIN DELINEATION

For

ACCOTINK CREEK BASIN

FAIRFAX COUNTY, VIRGINIA
Pat

$$
v_{c}^{G} 5
$$$$
\text { eroy }
$$

Open-File Report 76-442

Prepared in cooperation with the

County of Fairfax

1977

S. GEOLOGICAL SURVES
OCSTON, VA.
OCT $181978 \quad$ I BRAR 


\section{CONVERSION FACTORS}

Factors for converting English units to metric units are shown to four significant figures. However, in the text the metric equivalents are shown only to the number of significant figures consistent with the values for the English units.

\section{English}

$\begin{array}{ll}\text { acres } & 4.047 \times 10^{-3} \\ \begin{array}{l}\mathrm{ft} / \mathrm{s} \\ \text { (cubic feet per second) }\end{array} & 2.832 \times 10^{-2} \\ \mathrm{ft} \text { (feet) } & 3.048 \times 10^{-1} \\ \text { in (inches) } & 2.540 \times 10^{+1} \\ \mathrm{mi} \text { (miles) } & 1.609 \\ \mathrm{mi}^{2} \text { (square miles) } & 2.590\end{array}$

\section{Metric}

$\mathrm{km}^{2}$ (square kilometors)

$\mathrm{m}^{3} / \mathrm{s}$

(cubic meters per second)

m (meters)

mm (millimeters)

$\mathrm{km}$ (kilometers)

$\mathrm{km}^{2}$ (square kilometers) 
Abstract

Introduction

Purpose and scope

Acknow ledgments

Description of study area

Flood-plain mapping 17

Maps

Discharges

Profile computation 19

Delineation of flood boundaries

Flood profiles for Accotink Creek basin 22

Special hydraulic conditions

Flood-plain delineation for Accotink Creek basin

Selected references 
Figure 1. Location map of study area

Figure 2. Map of Accotink Creek basin 15

Figure 3-14 Graphs showing flood profiles for 25-year and 100year recurrence interval discharges

3-7 Accotink Creek $45-49$

8. Accotink Creek Tributary 50

9. Bear Branch 51

10. Long Branch near Vienna 52

11. Crook Branch 53

12. Turkey Run 54

13. Long Branch near Annandale 55

14. Long Branch near Newington 56

Figure 15. Index of flood maps 58

Figures 16-74 Topographic maps of stream valleys showing flood boundary delineation.

16-47 Accotink Creek; sheets AA 2-33 60

48-49 Accotink Creek Tributary; sheets AA2b \& 2c 92

50-51 Bear Branch; sheets AC $1 \& 2$

52-54 Long Branch near Vienna; sheets AD 1-3

55-56 Crook Branch; sheets AE $1 \& 2$

57-58 Turkey Run; sheets AF $1 \& 2$

59-67 Long Branch near Annandale; sheets AG 1-9 103

68-74 Long Branch near Newington; sheets AH 1-7 112 
Tables 1-8. Water-surface profile data for Accotink Creek basin.

1. Accotink Creek 25

2. Accotink Creek tributary 33

3. Bear Branch - 34

4. Long Branch near Vienna 35

5. Crook Branch 37

6. Turkey Run

7. Long Branch near Annandale 39

8. Long Branch near Newington 42 
Flood-Plain Delineation for Accotink Creek Basin

Fairfax County, Virginia

Pat L. Soule

ABSTRACT

Water-surface profiles of the 25-year and 100-year floods and maps on which the 25-, 50-, and 100-year flood limits are delineated for streams in the Accotink Creek basin are presented in this report. Excluded are segments of Accotink Creek within the Fort Belvoir Military Reservation.

The techniques used in the computation of the flood profiles and delineation of flood limits are presented, and specific hydraulic problems encountered within the study area are also included. 


\section{INTRODUCTION}

Suburban areas in many parts of the United States have experienced remarkable growth over the last decade or so. Much of this growth, which replaced farms and woodlands with streets, housing developments and shopping centers, caused serious environmental problems. Continued growth and increased competition for desirable space have required careful guidance and planning of future development to insure optimum land use. Fairfax County began to experience such growth during the early $1950^{\prime}$ s. In 1959 much of Fairfax County was still rural, but the desirability of regulating encroachment into the flood hazard areas had become apparent and appropriate local legislation was being enacted by Fairfax County. For the legislation to be effective, it was necessary to quantify the effect of development on floods and to delineate the boundaries of flood inundation.

The study of Accotink Creek basin, described in this report, was done by the U.S. Geological Survey in cooperation with Fairfax County. 
Data collection in Accotink Creek basin began in 1959, and the study continued through the release of maps and a letter report to the county of Fairfax in April 1970.

This report is one of a series summarizing the results of the hydraulic analysis done under the cooperative program between the county of Fairfax, the city of Alexandria and the Geological Survey. The hydrologic analysis has been published separately (Anderson, 1970). 


\section{Purpose and scope}

The purpose of the Accotink Creek study, was to determine the boundaries of floods having recurrence intervals of 25-,50-, and 100years under conditions of ultimate development. The flood boundaries were to be delineated on large scale maps that would become a part of the zoning ordinance documents for Fairfax County. The maps also could be used for management, design, and land-use planning.

To achieve the objective of the study, it was necessary to analyze the effects of urbanization upon floodflows and to develop techniques to compute the flood magnitude from measurable basin parameters for any given recurrence interval and for any degree of development. Reasonably accurate methods were available for estimating the magnitude and frequency of floods expected from drainage basins in a rural condition. However, as a drainage basin is changed from rural to a suburban or an urban condition, the magnitude and frequency of flooding also changes. Changes in flood frequency and magnitude resulting from basin development had received only scant study because of the sparse data available. 
The cooperative agreement between U.S. Geological Survey and Fairfax County, established a project to study the effects of basin development on floods and to delineate flood limits on specially prepared maps of stream valleys. The scope of the project included all of the basins in Fairfax County having a drainage area greater than 1 square mile. Excepted were the Dogue Creek and Little Hunting Creek basins, the upper tributaries of Cameron Run basin and Tripps Run and Holmes Run above Lake Barcroft. Studies of these basins were made by a private consulting firm. The maps were to have 2 foot $(0.60 \mathrm{~m})$ contours and be at a scale of 1 inch $(2.54 \mathrm{~cm})$ equals 100 feet $(30.5 \mathrm{~m})$. The project allowed for collection of basic data, for analytical investigation, and for definition of flood-prone areas. Anderson (1970) described the procedures used and the results obtained in the analysis of the effect of urbanization on flooding. He presented mathematical and graphical relations that may be used to estimate the flood discharge at a given recurrence interval up to 100 years for sites in the Washington Metropolitan area having various degrees of development. 
The purpose of this report is to provide a consolidated reference containing a summary of the techniques used in computation of flood profiles, a discussion of specific hydraulic problems encountered within the study area, a graphical presentation and listing of flood profiles, and the maps showing the area inundated by floods having recurrence intervals of 25-, 50-, and 100-years. 


\section{$\underline{\text { Acknowledgments }}$}

This report was prepared as a part of a cooperative agreement between Fairfax County and the U.S. Geological Survey. This report is a summary and compilation of data released to Fairfax County in the form of a letter report and on maps. The maps and profile tables presented in this report were prepared under the direction of F. Paul Kapinos of the Geological Survey. The base maps were provided by the U.S. Geological Survey. 


\section{Description of Study Area}

Fairfax County is in Northern Virginia adjacent to and just west of Washington, D. C. (Fig. 1) Fairfax County was formed by the division of the County of Prince William in 1742. From colonial days until World War II Fairfax County remained primarily rural. Following World War II, with the tremendous growth of Federal Government and influx of light industry, the County has lost its primary agricultural character and became largely residential. The population grew from 41,000 in 1940 to 564,000 in 1974 and it is estimated that by the year 2000 Fairfax County will have a population between 1 and 1.5 million. More than 50 percent of the 1 and area had been developed by 1974. (Written communication, Fairfax County, 1974).

The Accotink Creek basin is in the central part of Fairfax County between latitudes $38^{\circ} 40^{\prime} \mathrm{N}$ and $38^{\circ} 55^{\prime} \mathrm{N}$ and longitudes $77^{\circ} 08^{\prime} \mathrm{N}$ and $77^{\circ} 20^{\prime} \mathrm{W}$ (Fig. 2).

The basin has a southeasterly orientation with headwaters in the city of Fairfax and mouth at Gunston Cove on the Potomac River. The basin is bounded by Cameron Run and Dogue Creek basins to the east, Difficult Run to the north, and Popes Head Creek and Pohick Creek basins to the southwest. The entire drainage basin has a total area of approximately 51 square miles $\left(132 \mathrm{~km}^{2}\right)$. 


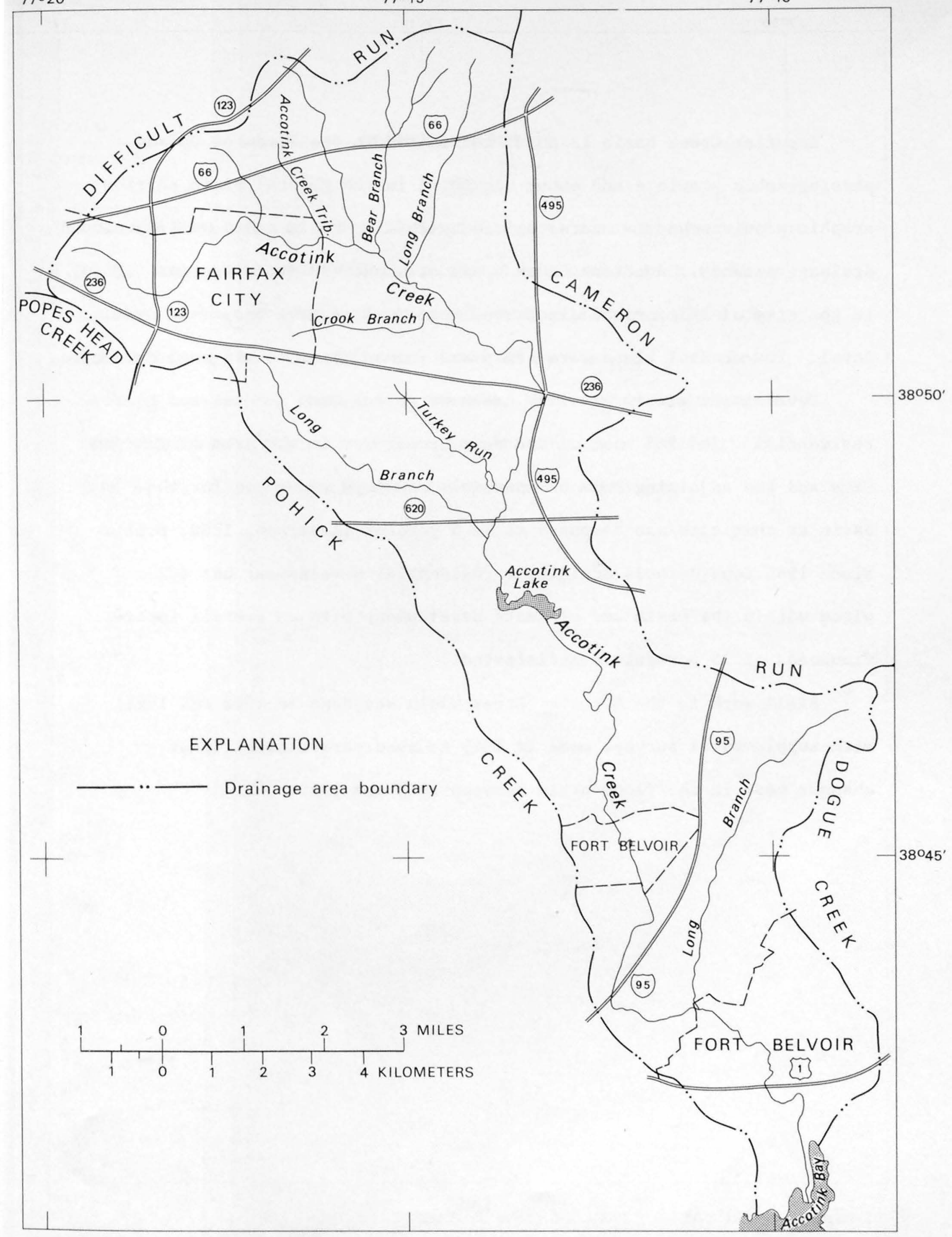

Figure 2.--Map of Accotink Creek basin 
Accotink Creek basin is about two thirds in the Piedmont Upland physiographic province and about one third in the Coastal Plain physiographic province and is characterized by rolling hills and a well developed drainage network. Accotink Creek heads at about $410 \mathrm{ft}$. elevation (125 m) in the city of Fairfax and discharges into Gunston Cove near mean sea leve1. The channel slopes are steep and stream channels are well developed. Development within Accotink Creek basin has been gradual and mostly residential. In 1965 most of the development was in the area of Fairfax City and the adjoining town of Annandale and imperviousness for the basin at that time was computed to be 5 percent (Anderson, 1970, p.11). Since 1965 considerable additional residential development has taken place within the basin and ultimate development with an overall imperviousness of 35 percent is anticipated.

Field work in the Accotink Creek basin was done in 1962 and 1963 with supplemental surveys made in 1969 to incorporate significant changes made in the flood plain between 1963 and 1969. 


\section{FLOOD-PLAIN MAPPING}

Maps.

The base maps on which flood limits are delineated have 2 -foot $(0.60)$ contour interval and a horizontal scale of 1 inch $(2.54 \mathrm{~cm})$ equals 100 feet $(30.5 \mathrm{~m})$. Natural and manmade features along the stream are shown. The maps were compiled by the U.S. Geological Survey, from aerial photographs taken in 1960 with revisions made from photographs taken in 1963. The maps include a 250-foot (76.2m) grid based on the Virginia coordinate system, north zone. 


\section{Discharges}

The flood areas delineated are those determined using ultimatedevelopment discharges. Highly developed basins differ from natural basins in that for a given storm (1) runoff is greater, (2) discharge time is much shorter, and (3) floods of comparable magnitude have a higher frequency of occurrence. These factors were analyzed through a study of streamflow and precipitation records, most of which were collected in the vicinity of Washington, D.C. The report, "Effects of Urban Development on Floods in Northern Virginia", (Anderson, 1970) describes the analysis used and summarizes the conclusions of that analysis. The effect of imperviousness with respect to runoff volumes was evaluated by comparing typical runoff coefficients for natural and highly developed basins. Regression analysis was used; first to derive the relation of lag time (the time lapse from the centroid of precipitation excess to the centroid of runoff) as a function of length-slope parameter; and second, to derive the relation of mean annual flood (2.33-year recurrence interval), adjusted for effects of imperviousness, as a function of drainage-basin area and lag time. An analysis of flood and rainfall frequencies was made to derive ratios of 25-, 50-, and 100-year floods to the mean annual flood for any percentage of imperviousness. Using the available information for a basin in the project area, the magnitude of the 2.33, 25-, 50-, and 100-year flood peaks can be computed from measurable basin parameters for any percentage of imperviousness in the ultimate development plan. 
The term recurrence interval, as used here, is the average interval of time within which a given flood discharge will be equaled or exceeded once. The recurrence interval is inversely related to the chance of a given flood being equaled or exceeded in any one year. Thus, the 100-year flood has a 1 percent chance of being equalled or exceeded in any one year. No periodicity is implied.

\section{Profile Computation}

Having determined the 25-, 50-, and 100-year floods computed as set forth by Anderson (1970) at selected points, the corresponding water-surface profiles were computed using the standard step method of backwater analysis. The method is based on a balance of energy between successive pairs of stream cross-sections. For tranquil flow the computations start at the farthest downstream section, or at a control section, and proceed upstream; but for supercritical flow the computations start at the most upstream section, or critical section, and proceed downstream until flow again becomes tranquil. Peak-discharge magnitudes were changed where necessary, such as above the confluence of a major tributary. The water-surface profile elevations are available for each cross-section. The cross-sections are referenced to an arbitrary base line, drawn to an approximate centroid of flow, and measured in an upstream direction from an arbitrary starting point. Profiles were computed in accordance with accepted methods of the U.S. Geological Survey. 
Following are several general items pertaining to the profile computations:

1. Discharge magnitudes greater than that of the 100-year flood may occur. However, the rate of change of stage per unit discharge generally becomes comparatively less as the discharge increases.

2. No factors of safety were used in the computations. Bridges and culverts were assumed to be free of debris. Roughness coefficients (Manning's " $n$ ") were selected based on summer vegetation.

3. New construction and channelization work may modify hydraulic properties thus changing the flood profiles in the future. 


\section{Delineation of Flood Boundaries}

The first step in the delineation of flood areas was the transposing of flood profile elevation, computed at each cross section, onto the base maps on which channel cross-section had initially been located. Delineation was then completed by interpreting elevation between these cross sections and between map contours on a straight-line basis.

The maps generally show the 25- and 100-year flood boundaries except in such areas where it is possible also to show the 50-year flood boundaries without confusion. In areas where topography is steep, there is insufficient space to show both the 25- and 100-year flood boundaries, and only the 100-year flood was delineated.

The delineation represents the average water-surface elevation. During actual floods, the water-surface may not be level across the stream. 
Flood Profile Data tables on Accotink Creek basin are:

Table 1. Water-surface profile data for Accotink Creek

Table 2. Water-surface profile data for Accotink Creek Tributary

Table 3. Water-surface profile data for Bear Branch.

Table 4. Water-surface profile data for Long Branch near Vienna

Table 5. Water-surface profile data for Crook Branch

Table 6. Water-surface profile data for Turkey Run

Table 7. Water-surface profile data for Long Branch near Annandale

Table 8. Water-surface profile data for Long Branch near Newington

The tables were prepared so that the reader can locate the position for

which information is desired on the flood plain maps included in this report, determine base-line stationing from the map by projection to the base line, and using the station number find in the table the nearest section for which information was determined.

For each cross section used in profile computations, tables 1 to 8 summarize: (1) The base-line reference stationing of that section, (2) the imperviousness determined from "Fairfax County, Map of Existing Zoning (Jan., 1963)" and County ultimate development plans used for computing discharge for the different recurrence interval flood at that point, (3) the resulting discharge and the water-surface profile elevations for 25-, 50-, and 100-year floods. 


\section{Special Hydraulic Conditions}

Special hydraulic conditions are discussed, beginning with Accotink Creek and then each tributary in the order of its listing.

1. Some channel changes (natural and man-made) have occurred in the Accotink Creek basin since the maps were prepared. The construction of new sewer lines along some portions of Accotink Creek and its tributaries has altered the topography along the stream channels. The profile computations were based on cross-sections surveyed prior to 1964 except where noted below.

2. The effect of Lake Accotink storage on reducing flood peaks in the lower reaches of Accotink Creek was studied and determined to be negligible.

3. There may be reverse flow from Accotink Creek through several tributary culverts under the Capital Beltway (Interstate 495) at the Little River Turnpike (State 236) interchange. Delineation of the areas within the cloverleafs and areas east of the interchange were not made because of project limitations.

4. A footbridge and sidewalk were constructed at Long Branch near Annandale just upstream of Braddock Road since the maps were made. The profile computations are for the conditions including the new footbridge. 
5. The flood elevations for Long Branch near Newington between Telegraph and Backlick Roads are approximate and subject to continuing changes due to the grave1 plant operations.

6. The culvert on Long Branch near Newington under the Fort Belvoir Military Railroad is inadequate to convey the 25-, 50-, and 100-year design peak discharges. The flood areas upstream and downstream of the railroad embankment were delineated without considering the present storage capacity upstream of the embankment that may reduce flood leve1s.

7. A maintenance yard was constructed on the left bank of Long Branch near Newington just upstream of Newington Road since the maps were made. The profile computations were based on the new cross-section properties which reflect the fill conditions existing after the maintenance yard was constructed. 
Table 1: Water-surface profile data for Accotink Creek

\begin{tabular}{|c|c|c|c|c|c|c|c|c|c|}
\hline $\begin{array}{l}\text { Section } \\
\text { number }\end{array}$ & $\begin{array}{c}\text { Base-line } \\
\text { Station } \\
\text { (feet) }\end{array}$ & $\begin{array}{l}\text { Impervi- } \\
\text { ousness } \\
\text { (per cent) }\end{array}$ & $\frac{\text { in } c u}{25-\text { year }^{a}}$ & $\begin{array}{l}\text { Discharge, } \\
\text { ic feet pe } \\
\frac{50 \text {-year }}{\mathrm{a}}\end{array}$ & $\frac{\mathrm{r} \text { second }}{100-\text { year }^{\mathrm{a}}}$ & 25 -yeara & $\begin{array}{l}\text { Elevation, } \\
\text { in feet } \\
50 \text {-yeara } \\
\end{array}$ & $100-y$ & Remarks \\
\hline 16 & $137+30$ & 35 & 13,400 & 16,700 & 20,000 & 44.4 & 44.8 & 45.2 & \\
\hline 17 & $143+20$ & 35 & 12,300 & 15,300 & 18,300 & 40.1 & 46.6 & 47.0 & \\
\hline 18 & $147+70$ & 35 & 12,300 & 15,300 & 18,300 & 47.4 & 48.0 & 48.4 & \\
\hline 19 & $150+80$ & 35 & 12,300 & 15,300 & 18,300 & 48.9 & 49.4 & 49.9 & \\
\hline 20 & $156+48$ & 35 & 12,300 & 15,300 & 18,300 & 50.1 & 50.7 & 51.2 & \\
\hline 21 & $157+00$ & & & & & & & & Telegraph Road (State 611) \\
\hline 22 & $157+35$ & 35 & 12,300 & 15,300 & 18,300 & 50.1 & 50.8 & 51.3 & \\
\hline 23 & $163+86$ & 35 & 12,300 & 15,300 & 18,300 & 51.6 & 52.3 & 52.8 & \\
\hline 24 & $170+69$ & 35 & 12,300 & 15,300 & 18,300 & 53.1 & 53.6 & 54.1 & \\
\hline 25 & $177+72$ & 35 & 12,300 & 15,300 & 18,300 & 55.5 & 55.8 & 56.1 & \\
\hline 26 & $187+32$ & 35 & 10,000 & 12,400 & 14,700 & 57.8 & 58.2 & 58.6 & By-Pass Flow at Left Bank \\
\hline 27 & $195+65$ & 35 & 10,000 & 12,400 & 14.700 & 60.2 & 60.7 & 61.2 & By-Pass Flow at Left Bank \\
\hline 28 & $203+00$ & 35 & 10,000 & 12,400 & 14,700 & 61.8 & 62.5 & 63.1 & By-Pass Flow at Left Bank \\
\hline 29 & $203+90$ & & & & & & & & R.F. \& P. Railroad Bridge \\
\hline 31 & $205+49$ & 35 & 12,300 & 15,300 & 18,300 & 68.0 & 70.8 & 73.4 & \\
\hline 32 & $210+68$ & 35 & 12,300 & 15,300 & 18,300 & 68.0 & 70.8 & 73.4 & \\
\hline 33 & $211+87$ & & & & & & & & Shirley Hwy (I-95) \\
\hline 34 & $213+59$ & 35 & 9,100 & 9,800 & 9,800 & 71.4 & 72.4 & 73.5 & By-Pass Flow on Left Bank \\
\hline 35 & $216+23$ & 35 & 10,800 & 12,900 & 14,000 & 71.7 & 72.6 & 73.7 & By-Pass Flow on Left Bank \\
\hline 36 & $220+13$ & 35 & 12,300 & 15,300 & 18,100 & 72.0 & 73.0 & 74.0 & By-Pass Flow on Left Bank \\
\hline 37 & $225+45$ & 35 & 12,300 & 15,300 & 18,300 & 72.3 & 73.3 & 74.4 & \\
\hline 38 & $225+60$ & & & & & & & & Alban Road (State 790) \\
\hline 39 & $226+71$ & 35 & 12,300 & 15,300 & 18,300 & 72.8 & 73.9 & 74.9 & \\
\hline 40 & $229+16$ & 35 & 12,300 & 15,300 & 18,300 & 73.0 & 74.0 & 75.1 & \\
\hline 41 & $234+02$ & 35 & 12,300 & 15,300 & 18,300 & 73.5 & 74.7 & 75.8 & \\
\hline 42 & $238+39$ & 35 & 12,300 & 15,300 & 18,300 & 73.9 & 75.1 & 76.2 & \\
\hline 43 & $241+31$ & 35 & 12,300 & 15,300 & 18,300 & 74.2 & 75.5 & 76.6 & \\
\hline 44 & $244+53$ & 35 & 12,300 & 15,300 & 18,300 & 74.7 & 76.0 & 77.1 & \\
\hline 45 & $247+85$ & 35 & 12,300 & 15,300 & 18,300 & 75.5 & 76.8 & 78.1 & \\
\hline 46 & $250+41$ & 35 & 12,300 & 15,300 & 18,300 & 76.5 & 78.0 & 79.3 & \\
\hline 47 & $253+62$ & 35 & 12,300 & 15,300 & 18,300 & 77.7 & 79.3 & 80.7 & \\
\hline
\end{tabular}


Accotink Creek Basin

Table 1: Water-surface profile data for Accotink Creek - Continued

\begin{tabular}{|c|c|c|c|c|c|c|c|c|c|}
\hline $\begin{array}{l}\text { Section } \\
\text { number }\end{array}$ & $\begin{array}{c}\text { Base-1ine } \\
\text { station } \\
\text { (feet) }\end{array}$ & $\begin{array}{l}\text { Impervi- } \\
\text { ousness } \\
\text { (per cent) }\end{array}$ & $\frac{\text { in cub }}{25-\text { year }^{\mathrm{a}}}$ & $\begin{array}{l}\text { Discharge, } \\
\text { c feet per } \\
50 \text {-yeara }\end{array}$ & $\frac{\text { second }}{100-\text { year }^{a}}$ & 25 -year & $\begin{array}{l}\text { Elevation } \\
\text { in feet } \\
50 \text {-year } \\
\end{array}$ & $100-y^{2} a r^{2}$ & Remarks \\
\hline 48 & $256+22$ & 35 & 12,300 & 15,300 & 18,300 & 78.9 & 80.5 & 81.9 & \\
\hline 49 & $258+72$ & 35 & 12,300 & 15,300 & 18,300 & 79.8 & 81.4 & 82.8 & \\
\hline 50 & $260+46$ & 35 & 12,300 & 15,300 & 18,300 & 80.4 & 82.0 & 83.5 & \\
\hline 51 & $262+67$ & 35 & 12,300 & 15,300 & 18,300 & 81.1 & 82.7 & 84.2 & \\
\hline 52 & $265+48$ & 35 & 12,300 & 15,300 & 18,300 & 82.0 & 83.6 & 85.1 & \\
\hline 53 & $268+98$ & 35 & 12,300 & 15,300 & 18,300 & 84.0 & 85.9 & 87.5 & \\
\hline 54 & $272+30$ & 35 & 12,300 & 15,300 & 18,300 & 85.0 & 86.8 & 88.4 & \\
\hline 55 & $274+43$ & 35 & 12,300 & 15,300 & 18,300 & 86.1 & 88.0 & 89.6 & \\
\hline 56 & $276+04$ & 35 & 12,300 & 15,300 & 18,300 & 86.2 & 88.1 & 89.8 & \\
\hline 57 & $278+17$ & 35 & 12,300 & 15,300 & 18,300 & 86.6 & 88.4 & 90.0 & \\
\hline 58 & $281+60$ & 35 & 12,300 & 15,300 & 18,300 & 89.3 & 90.9 & 92.4 & \\
\hline 59 & $284+70$ & 35 & 12,300 & 15,300 & 18,300 & 91.9 & 93.4 & 94.8 & \\
\hline 60 & $286+55$ & 35 & 12,300 & 15,300 & 18,300 & 93.8 & 95.4 & 96.8 & \\
\hline 61 & $288+68$ & 35 & 12,300 & 15,300 & 18,300 & 95.7 & 97.1 & 98.4 & \\
\hline 62 & $290+62$ & 35 & 12,300 & 15,300 & 18,300 & 98.5 & 99.9 & 101.2 & \\
\hline 63 & $291+78$ & 35 & 12,300 & 15,300 & 18,300 & 99.9 & 101.3 & 102.6 & \\
\hline 64 & $293+13$ & 35 & 12,300 & 15,300 & 18,300 & 102.6 & 104.1 & 105.5 & \\
\hline 65 & $294+60$ & 35 & 12,300 & 15,300 & 18,300 & 104.7 & 106.4 & 107.9 & \\
\hline 66 & $296+88$ & 35 & 12,300 & 15,300 & 18,300 & 106.4 & 108.1 & 109.6 & \\
\hline 67 & $299+67$ & 35 & 12,300 & 15,300 & 18,300 & 108.4 & 110.0 & 111.4 & \\
\hline \multirow[t]{2}{*}{68} & $302+43$ & 35 & 12,300 & 15,300 & 18,300 & 110.8 & 112.5 & 114.1 & \\
\hline & $302+70$ & & & & & & & & $\begin{array}{l}\text { voir Military } \\
\text { tion Boundary }\end{array}$ \\
\hline
\end{tabular}


Table 1: Water-surface profile data for Accotink Creek - Continued

\begin{tabular}{|c|c|c|c|c|c|c|c|c|c|}
\hline \multirow{2}{*}{$\begin{array}{l}\text { Section } \\
\text { number }\end{array}$} & \multirow{2}{*}{$\begin{array}{c}\text { Base-1ine } \\
\text { Station } \\
\text { (feet) }\end{array}$} & \multirow{2}{*}{$\begin{array}{l}\text { Impervi } \\
\text { ousness } \\
\text { (per cent) }\end{array}$} & \multicolumn{3}{|c|}{$\begin{array}{l}\text { Discharge, } \\
\text { ubic feet per second }\end{array}$} & \multicolumn{3}{|c|}{$\begin{array}{l}\text { Elevation, } \\
\text { in feet }\end{array}$} & \multirow[t]{2}{*}{ Remarks } \\
\hline & & & 25 -year ${ }^{\mathrm{a}}$ & $50-$ yeara $^{a}$ & $100-$ yeara $^{a}$ & 25 -yeara & 50 -yeara & 100 -year & \\
\hline & $400+00$ & & & & & & & & $\begin{array}{l}\text { Fort Belvoir Military } \\
\text { Reservation Boundary }\end{array}$ \\
\hline 100 & $400+32$ & 35 & 12,000 & 14,900 & 17,900 & 145.5 & 146.9 & 147.9 & \\
\hline 101 & $402+13$ & 35 & 12,000 & 14,900 & 17,900 & 145.8 & 147.2 & 148.3 & \\
\hline 102 & $404+29$ & 35 & 12,000 & 14,900 & 17,900 & 145.9 & 147.4 & 148.5 & \\
\hline 103 & $406+88$ & 35 & 12,000 & 14,900 & 17,900 & 146.1 & 147.7 & 148.8 & \\
\hline 104 & $409+03$ & 35 & 12,000 & 14,900 & 17,900 & 146.3 & 147.9 & 149.1 & \\
\hline 105 & $411+54$ & 35 & 12,000 & 14,900 & 17,900 & 146.5 & 148.0 & 149.2 & \\
\hline 106 & $413+11$ & 35 & 12,000 & 14,900 & 17,900 & 146.8 & 148.3 & 149.6 & \\
\hline 107 & $414+73$ & 35 & 12,000 & 14,900 & 17,900 & 147.0 & 148.6 & 150.0 & \\
\hline 108 & $416+77$ & 35 & 12,000 & 14,900 & 17,900 & 147.5 & 149.3 & 150.7 & \\
\hline 109 & $418+84$ & 35 & 12,000 & 14,900 & 17,900 & 147.8 & 149.6 & 151.1 & \\
\hline 110 & $421+45$ & 35 & 12,000 & 14,900 & 17,900 & 148.0 & 149.8 & 151.3 & \\
\hline 111 & $423+65$ & 35 & 12,000 & 14,900 & 17,900 & 148.2 & 150.0 & 151.6 & \\
\hline 112 & $425+44$ & 35 & 12,000 & 14,900 & 17,900 & 148.8 & 150.7 & 152.3 & \\
\hline 113 & $426+61$ & 35 & 12,000 & 14,900 & 17,900 & 149.0 & 150.8 & 152.5 & \\
\hline 114 & $427+26$ & 35 & 12,000 & 14,900 & 17,900 & 149.0 & 150.9 & 152.6 & \\
\hline $114 \mathrm{~A}$ & $427+50$ & & & & & & & & Hooes Road (State 636) \\
\hline 115 & $428+14$ & 35 & 12,000 & 14,900 & 17,900 & 149.1 & 151.0 & 152.7 & \\
\hline 116 & $429+26$ & 35 & 12,000 & 14,900 & 17,900 & 149.2 & 151.1 & 152.8 & \\
\hline 117 & $431+20$ & 35 & 12,000 & 14,900 & 17,900 & 149.7 & 151.6 & 153.3 & \\
\hline 118 & $432+95$ & 35 & 12,000 & 14,900 & 17,900 & 149.9 & 151.8 & 153.5 & \\
\hline 119 & $434+89$ & 35 & 12,000 & 14,900 & 17,900 & 150.2 & 152.1 & 153.9 & \\
\hline 120 & $437+76$ & 35 & 12,000 & 14,900 & 17,900 & 150.5 & 152.4 & 154.2 & \\
\hline 121 & $440+66$ & 35 & 12,000 & 14,900 & 17,900 & 151.0 & 152.8 & 154.6 & \\
\hline 122 & $443+75$ & 35 & 12,000 & 14,900 & 17,900 & 151.5 & 153.4 & 155.2 & \\
\hline 123 & $447+49$ & 35 & 12,000 & 14,900 & 17,900 & 152.0 & 153.9 & 155.7 & \\
\hline 124 & $449+77$ & 35 & 12,000 & 14,900 & 17,900 & 152.2 & 154.2 & 156.0 & \\
\hline 125 & $452+71$ & 35 & 12,000 & 14,900 & 17,900 & 152.5 & 154.5 & 156.3 & \\
\hline 126 & $454+84$ & 35 & 12,000 & 14,900 & 17,900 & 152.9 & 154.9 & 156.6 & \\
\hline 127 & $459+63$ & 35 & 12,000 & 14,900 & 17,900 & 153.5 & 155.4 & 157.1 & \\
\hline 128 & $463+99$ & 35 & 12,000 & 14,900 & 17,900 & 154.0 & 155.8 & 157.6 & \\
\hline
\end{tabular}


Accotink Creek Basin

Table 1: Water-surface profile data for Accotink Creek - Continued

Base-line Impervi- Discharge, Elevation,

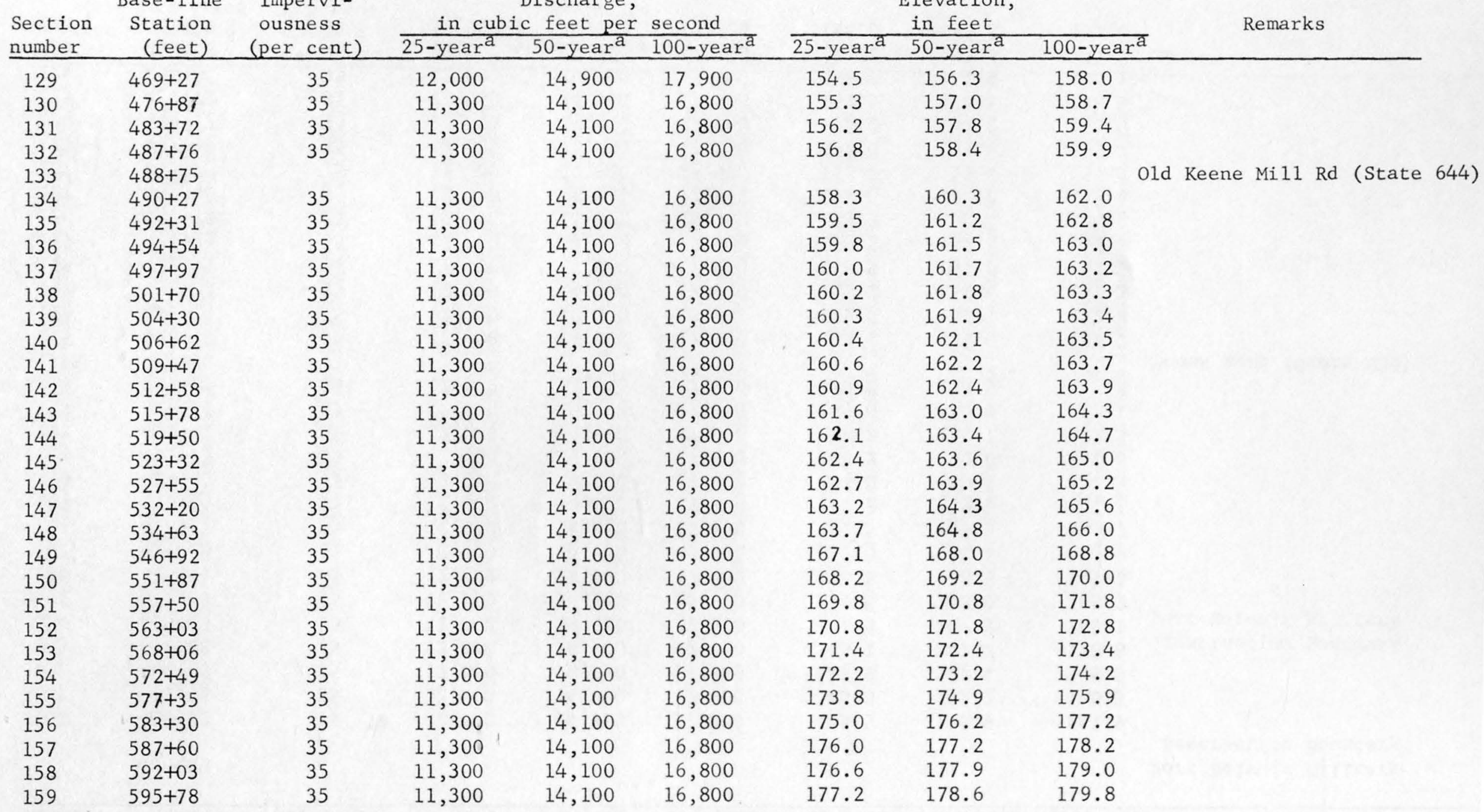


Accotink Creek Basin

Table 1: Water-surface profile data for Accotink Creek - Continued

Base-line Impervi- Discharge, Elevation,

\begin{tabular}{|c|c|c|c|c|c|c|c|c|c|}
\hline \multirow{2}{*}{$\begin{array}{l}\text { Section } \\
\text { number }\end{array}$} & \multirow{2}{*}{$\begin{array}{c}\text { Station } \\
\text { (feet) }\end{array}$} & \multirow{2}{*}{$\begin{array}{l}\text { ousness } \\
\text { (per cent) }\end{array}$} & \multicolumn{3}{|c|}{ in cubic feet per second } & \multicolumn{3}{|c|}{ in feet } & \multirow[t]{2}{*}{ Remarks } \\
\hline & & & $25-$ year $^{a}$ & $50-$ year $^{\mathrm{a}}$ & $100-$ year $^{2}$ & 25 -year ${ }^{2}$ & $50-$ year $^{2}$ & 100 -year & \\
\hline 160 & $598+89$ & 35 & 11,300 & 14,100 & 16,800 & 177.6 & 179.0 & 180.1 & \\
\hline 161 & $602+22$ & 35 & 11,300 & 14,100 & 16,800 & 178.0 & 179.5 & 180.6 & \\
\hline 162 & $606+92$ & 35 & 11,300 & 14,100 & 16,800 & 178.5 & 179.9 & 181.0 & \\
\hline 163 & $610+69$ & 35 & 11,300 & 14,100 & 16,800 & 179.1 & 180.5 & 181.6 & \\
\hline 164 & $612+76$ & 35 & 11,300 & 14,100 & 16,800 & 179.6 & 181.0 & 182.1 & \\
\hline 165 & $613+00$ & & & & & & & & Southern Railway Trestle \\
\hline 166 & $613+73$ & 35 & 11,300 & 14,100 & 16,800 & 180.1 & 181.5 & 182.6 & \\
\hline 167 & $615+00$ & & & & & & & & Lake Accotink Spillway \\
\hline $68-198$ & & & & & & 191.1 & 191.8 & 192.5 & Lake Accotink \\
\hline 199 & $644+30$ & 35 & 10,800 & 13,700 & 16,400 & 191.6 & 192.3 & 193.1 & \\
\hline 200 & $650+86$ & 35 & 10,800 & 13,700 & 16,400 & 192.0 & 192.8 & 193.5 & \\
\hline 201 & $659+25$ & 35 & 10,800 & 13,700 & 16,400 & 192.5 & 193.2 & 194.0 & \\
\hline 202 & $664+77$ & 35 & 10,800 & 13,700 & 16,400 & 193.9 & 194.7 & 195.5 & \\
\hline 203 & $669+40$ & 35 & 10,800 & 13,700 & 16,400 & 195.6 & 196.6 & 197.4 & \\
\hline 204 & $676+12$ & 35 & 10,800 & 13,700 & 16,400 & 196.7 & 197.7 & 198.5 & \\
\hline 205 & $683+08$ & 35 & 10,800 & 13,700 & 16,400 & 198.0 & 199.0 & 199.8 & \\
\hline 206 & $688+11$ & 35 & 10,800 & 13,700 & 16,400 & 198.8 & 199.8 & 200.6 & \\
\hline 207 & $693+05$ & 35 & 10,800 & 13,700 & 16,400 & 199.8 & 200.8 & 201.6 & \\
\hline 208 & $698+11$ & 35 & 10,800 & 13,700 & 16,400 & 201.1 & 202.0 & 202.8 & \\
\hline 209 & $702+92$ & 35 & 10,800 & 13,700 & 16,400 & 201.9 & 202.8 & 203.6 & \\
\hline 210 & $708+81$ & 35 & 10,800 & 13,700 & 16,400 & 202.5 & 203.3 & 204.0 & \\
\hline 211 & $709+08$ & & & & & & & & Braddock Road (State 620) \\
\hline 212 & $709+98$ & 35 & 9,500 & 11,800 & 14,100 & 202.7 & 203.5 & 204.1 & \\
\hline 213 & $717+34$ & 35 & 9,500 & 11,800 & 14,100 & 204.2 & 204.7 & 205.2 & \\
\hline 214 & $722+47$ & 35 & 9,500 & 11,800 & 14,100 & 205.5 & 206.2 & 206.8 & \\
\hline 215 & $731+12$ & 35 & 9,500 & 11,800 & 14,100 & 207.1 & 207.8 & 208.4 & \\
\hline 216 & $736+91$ & 35 & 9,200 & 11,300 & 13,500 & 207.9 & 208.6 & 209.3 & \\
\hline 217 & $742+13$ & 35 & 9,200 & 11,300 & 13,500 & 209.0 & 209.8 & 210.4 & \\
\hline 218 & $746+64$ & 35 & 9,200 & 11,300 & 13,500 & 210.2 & 210.9 & 211.6 & \\
\hline 219 & $752+66$ & 35 & 9,200 & 11,300 & 13,500 & 211.6 & 212.2 & 213.0 & \\
\hline 220 & $755+60$ & 35 & 9,200 & 11,300 & 13,500 & 212.5 & 213.2 & 214.0 & \\
\hline
\end{tabular}




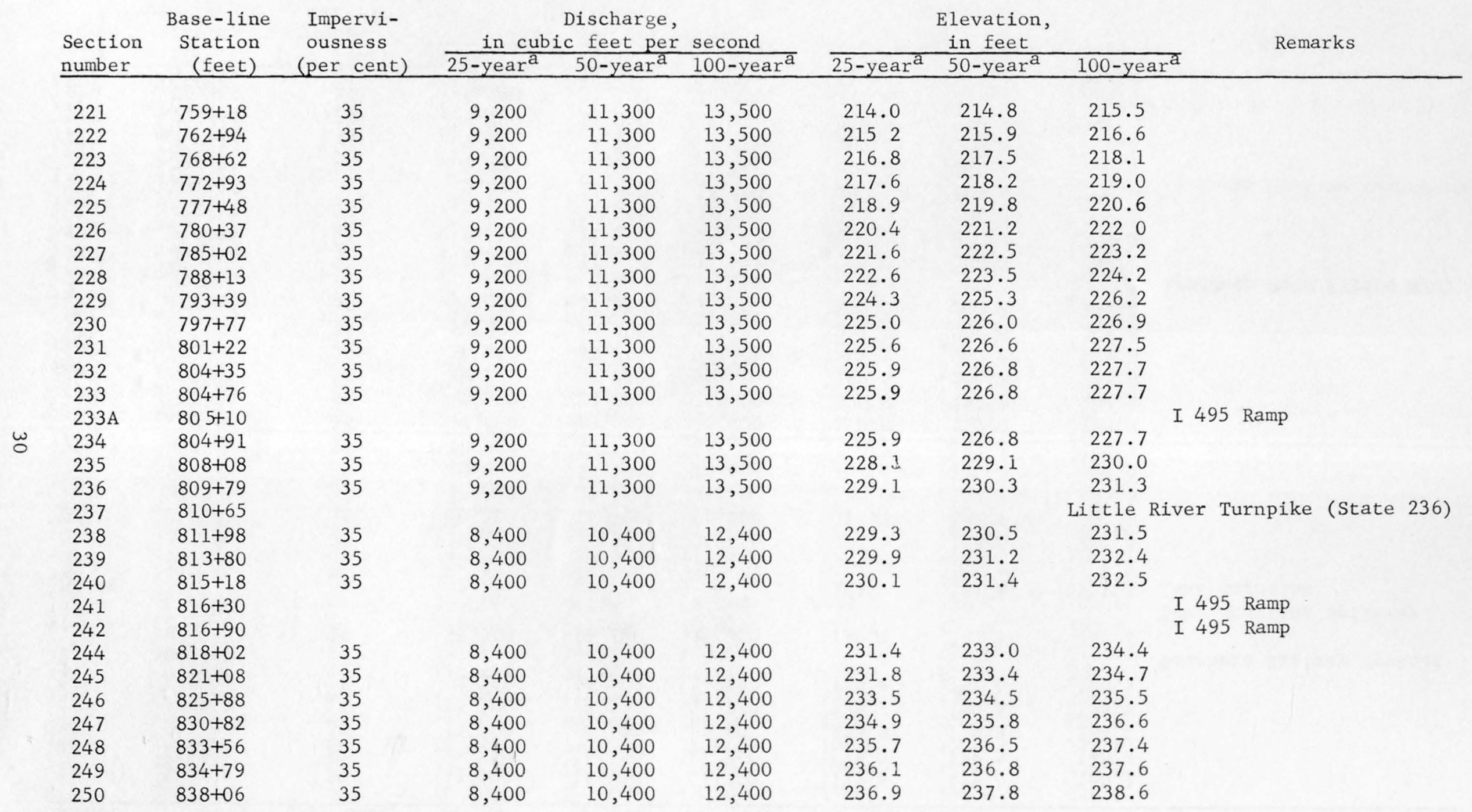


Accotink Creek Basin

Table 1: Water-surface profile data for hccotink Creek - Continued

\begin{tabular}{|c|c|c|c|c|c|c|c|c|c|}
\hline $\begin{array}{l}\text { Section } \\
\text { number }\end{array}$ & $\begin{array}{c}\text { Base-line } \\
\text { Station } \\
\text { (feet) }\end{array}$ & $\begin{array}{l}\text { Impervi- } \\
\text { ousness } \\
\text { (per cent) }\end{array}$ & $\frac{\text { in cub }}{25-\text { year }^{a}}$ & $\begin{array}{l}\text { Discharge, } \\
\text { c feet per } \\
50 \text {-yeara }\end{array}$ & $\frac{\text { second }}{100 \text {-year }}$ & 25 -year ${ }^{2}$ & $\begin{array}{c}\begin{array}{c}\text { Elevation, } \\
\text { in feet }\end{array} \\
50 \text {-year } \\
\end{array}$ & 100 -yea & Remarks \\
\hline 251 & $840+14$ & 35 & 8,400 & 10,400 & 12,400 & 237.6 & 238.5 & 239.2 & \\
\hline 252 & $841+95$ & 35 & 8,400 & 10,400 & 12,400 & 238.2 & 239.2 & 240.2 & \\
\hline 253 & $845+23$ & 35 & 8,400 & 10,400 & 12,400 & 238.9 & 239.9 & 240.9 & \\
\hline 254 & $848+08$ & 35 & 8,400 & 10,400 & 12,400 & 239.8 & 240.8 & 241.8 & \\
\hline 255 & $850+55$ & 35 & 8,400 & 10,400 & 12,400 & 240.9 & 241.8 & 242.7 & \\
\hline 256 & $853+14$ & 35 & 8,400 & 10,400 & 12,400 & 242.3 & 243.2 & 244.0 & \\
\hline 257 & $855+35$ & 35 & 8,400 & 10,400 & 12,400 & 242.9 & 243.8 & 244.6 & \\
\hline 258 & $858+55$ & 35 & 8,400 & 10,400 & 12,400 & 244.0 & 245.0 & 245.9 & \\
\hline 259 & $861+54$ & 35 & 8,400 & 10,400 & 12,400 & 244.6 & 245.6 & 246.5 & \\
\hline 260 & $863+89$ & 35 & 8,400 & 10,400 & 12,400 & 245.4 & 246.4 & 247.2 & \\
\hline 261 & $866+58$ & 35 & 8,400 & 10,400 & 12,400 & 247.0 & 247.8 & 248.4 & \\
\hline 262 & $869+35$ & 35 & 8,400 & 10,400 & 12,400 & 248.1 & 248.8 & 249.5 & \\
\hline 263 & $872+39$ & 35 & 8,400 & 10,400 & 12,400 & 248.8 & 249.6 & 250.2 & \\
\hline 264 & $874+52$ & 35 & 8,400 & 10,400 & 12,400 & 249.2 & 250.2 & 251.0 & \\
\hline 265 & $879+42$ & 35 & 8,400 & 10,400 & 12,400 & 250.0 & 251.0 & 251.8 & \\
\hline 266 & $883+22$ & 35 & 8,400 & 10,400 & 12,400 & 250.4 & 251.4 & 252.2 & \\
\hline $266 \mathrm{~A}$ & $886+00$ & 35 & 8,400 & 10,400 & 12,400 & 251.1 & 252.0 & 252.8 & \\
\hline 267 & $886+47$ & & & & & & & & King Arthur Road \\
\hline 268 & $887+95$ & 35 & 8,400 & 10,400 & 12,400 & 251.9 & 253.0 & 253.9 & \\
\hline $268 A$ & $889+46$ & 35 & 8,400 & 10,400 & 12,400 & 252.4 & 253.5 & 254.4 & \\
\hline 269 & $892+31$ & 35 & 8,400 & 10,400 & 12,400 & 252.8 & 253.9 & 254.8 & \\
\hline 270 & $895+93$ & 35 & 8,400 & 10,400 & 12,400 & 253.4 & 254.5 & 255.4 & \\
\hline 271 & $898+94$ & 35 & 8,400 & 10,400 & 12,400 & 253.6 & 254.8 & 255.6 & \\
\hline 272 & $902+46$ & 35 & 8,400 & 10,400 & 12,400 & 254.0 & 255.1 & 256.0 & \\
\hline 273 & $906+50$ & 35 & 8,400 & 10,400 & 12,400 & 254.3 & 255.4 & 256.3 & \\
\hline 274 & $910+01$ & 35 & 8,400 & 10,400 & 12,400 & 254.6 & 255.7 & 256.5 & \\
\hline 275 & $912+82$ & 35 & 8,400 & 10,400 & 12,400 & 255.2 & 256.2 & 257.0 & \\
\hline 276 & $914+60$ & 35 & 8,400 & 10,400 & 12,400 & 255.8 & 256.8 & 257.6 & \\
\hline 277 & $916+32$ & 35 & 8,400 & 10,400 & 12,400 & 255.9 & 256.9 & 257.8 & \\
\hline 278 & $916+60$ & & & & & & & & Woodburn Road (State 650) \\
\hline 279 & $917+04$ & 35 & 8,400 & 10,400 & 12,400 & 256.0 & 257.0 & 257.8 & \\
\hline
\end{tabular}


Table I: Water-surface profile data for Accotink Cresk - Continued

Base-1ine Impervinumber (feet) (per cent)

\begin{tabular}{lr}
280 & $919+33$ \\
281 & $926+56$ \\
282 & $930+91$ \\
283 & $936+99$ \\
284 & $943+30$ \\
285 & $947+68$ \\
286 & $949+28$ \\
287 & $950+78$ \\
288 & $953+85$ \\
289 & $956+75$ \\
290 & $962+31$ \\
291 & $962+76$ \\
292 & $963+41$ \\
293 & $968+52$ \\
294 & $977+06$ \\
295 & $984+39$ \\
296 & $990+71$ \\
297 & $995+35$ \\
298 & $998+28$ \\
299 & $998+80$ \\
300 & $999+27$ \\
301 & $1004+74$ \\
302 & $1008+12$ \\
303 & $1015+62$ \\
$303 A$ & $1022+82$ \\
304 & $1027+18$ \\
305 & $1033+90$ \\
306 & $1041+82$ \\
307 & $1048+30$ \\
& $1048+60$ \\
& $1058+05$ \\
\hline 30 & \\
295
\end{tabular}

Discharge, $\frac{\text { in cubic feet per second }}{25 \text {-year } \text { y }^{a} \text {-year }}$

8,400

$$
7,800
$$

$$
7,800
$$$$
7,800
$$$$
7,800
$$$$
7,800
$$$$
7,800
$$$$
7,800
$$$$
7,800
$$$$
7,800
$$

$$
7,800
$$

$$
7,800
$$$$
7,800
$$$$
7,800
$$$$
6,700
$$

$$
6,700
$$$$
6,700
$$

$$
6,700
$$

$$
6,700
$$$$
6,700
$$$$
5,800
$$$$
5,800
$$$$
\text { 5, } 000
$$$$
\text { 5, } 000
$$$$
\text { 5, } 000
$$$$
\text { 5, } 000
$$

5, 000

50

$\begin{array}{rr}10,400 & 12,400 \\ 9,700 & 11,600 \\ 9,700 & 11,600 \\ 9,700 & 11,600 \\ 9,700 & 11,600 \\ 9,700 & 11,600 \\ 9,700 & 11,600 \\ 9,700 & 11,600 \\ 9,700 & 11,600 \\ 9,700 & 11,600 \\ 9,700 & 11,600\end{array}$

$9,700 \quad 11,600$

$9,700 \quad 11,600$

$9,700 \quad 11,600$

$8,200 \quad 9,900$

$8,200 \quad 9,900$

$8,200 \quad 9,900$

$8,200 \quad 9,900$

25-year ${ }^{\text {a }}$ 5

Elevation,

in feet $\quad$ Remarks

\section{2}

257.2

$256.8-257.8 \quad 258.0$

$\begin{array}{lll}257.3 & 258.2 & 258.5\end{array}$

(

$\begin{array}{lll}259.9 & 260.5 & 261.1\end{array}$

$261.7 \quad 262.3 \quad 262.9$

$262.2 \quad 262.9 \quad 263.5$

$\begin{array}{lll}263.1 & 263.8 & 264.5\end{array}$

$263.5 \quad 264.3 \quad 265.0$

$\begin{array}{lll}263.6 & 264.4 & 265.1\end{array}$

$\begin{array}{lll}263.9 & 264.7 & 265.4\end{array}$

264.5

$265.0 \quad 265.5 \quad 266.1$

265.0

265.6

$266.1 \quad 266.6 \quad 267.1$

$267.7 \quad 268.0 \quad 268.4$

$\begin{array}{lll}270.1 & 270.5 & 270.9\end{array}$

$\begin{array}{lll}272.4 & 272.8 & 273.2\end{array}$

$\begin{array}{lll}273.0 & 273.5 & 274.1\end{array}$

275.5

$\begin{array}{lllll}8,200 & 9,900 & 275.5 & 275.8 & 276.2 \\ 8,200 & 9,900 & 275.7 & 276.0 & 276.4 \\ 7,100 & 8,500 & 275.9 & 276.2 & 276.6 \\ 7,100 & 8,500 & 276.7 & 277.0 & 277.4 \\ 6,200 & 7,400 & 277.8 & 278.1 & 278.6 \\ 6,200 & 7,400 & 278.4 & 278.8 & 279.3 \\ 6,200 & 7,400 & 280.2 & 280.5 & 280.9 \\ 6,200 & 7,400 & 283.0 & 283.4 & 283.7\end{array}$

Prosperity Avenue (State 699)

\section{Barkley Drive}

Pickett Street 
Accotink Creek Basin

Table 2: Water-surface profile data for Accotink Creek Tributary

Base-line Impervi- Discharge, Elevation,

\begin{tabular}{|c|c|c|c|c|c|c|c|c|c|}
\hline \multirow{2}{*}{$\begin{array}{l}\text { Section } \\
\text { number }\end{array}$} & \multirow{2}{*}{$\begin{array}{c}\text { Station } \\
(\text { feet })\end{array}$} & \multirow{2}{*}{$\begin{array}{c}\text { ousness } \\
\text { (percent) }\end{array}$} & \multicolumn{3}{|c|}{ in cubic feet per second } & \multicolumn{3}{|c|}{ in feet } & \multirow[t]{2}{*}{ Remarks } \\
\hline & & & 25 -yeara & $50-$ year $^{a}$ & $100-$ yeara $^{2}$ & 25 -year ${ }^{a}$ & $50-$ year $^{a}$ & $100-$ year & \\
\hline 898 & $6+50$ & 30 & 1,700 & 2,100 & 2,500 & 277.2 & 277.6 & 278.0 & \\
\hline 899 & $7+96$ & 30 & 1,700 & 2,100 & 2,500 & 279.4 & 279.6 & 279.7 & \\
\hline 900 & $10+00$ & 30 & 1,700 & 2,100 & 2,500 & 280.6 & 280.8 & 281.1 & \\
\hline 901 & $13+90$ & 30 & 1,700 & 2,100 & 2,500 & 284.2 & 284.6 & 284.9 & \\
\hline 902 & $15+70$ & 30 & 1,700 & 2,100 & 2,500 & 285.0 & 285.4 & 285.7 & \\
\hline 903 & $16+37$ & & & & & & & & Arlington Blvd. (US 50) \\
\hline 904 & $17+16$ & 30 & 1,700 & 2,100 & 2,500 & 288.6 & 289.0 & 289.4 & \\
\hline 905 & $20+59$ & 30 & 1,700 & 2,100 & 2,500 & 288.8 & 289.1 & 289.5 & \\
\hline 906 & $25+40$ & 30 & 1,700 & 2,100 & 2,500 & 289.3 & 289.7 & 290.0 & \\
\hline 907 & $29+97$ & 30 & 1,700 & 2,100 & 2,500 & 292.3 & 292.6 & 292.8 & \\
\hline 908 & $34+18$ & 30 & 1,700 & 2,100 & 2,500 & 295.3 & 295.6 & 295.8 & \\
\hline 909 & $36+89$ & 30 & 1,700 & 2,100 & 2,500 & 297.0 & 296.4 & 297.6 & \\
\hline 910 & $40+59$ & 30 & 1,700 & 2,100 & 2,500 & 300.5 & 300.8 & 301.2 & \\
\hline 911 & $43+45$ & 30 & 1,700 & 2,100 & 2,500 & 301.8 & 302.2 & 302.5 & \\
\hline 912 & $44+07$ & & & & & & & & Lee Highway (US 29-211) \\
\hline 913 & $44+57$ & 30 & 1,400 & 1,800 & 2,100 & 305.9 & 306.3 & 306.6 & \\
\hline 914 & $47+79$ & 30 & 1,400 & 1,800 & 2,100 & 306.0 & 306.4 & 306.8 & \\
\hline 915 & $51+75$ & 30 & 1,400 & 1,800 & 2,100 & 306.5 & 306.9 & 307.3 & \\
\hline 916 & $54+54$ & 30 & 1,400 & 1,800 & 2,100 & 307.2 & 307.7 & 308.1 & \\
\hline 917 & $57+27$ & 30 & 1,400 & 1,800 & 2,100 & 308.4 & 308.8 & 309.2 & \\
\hline 918 & $59+85$ & 30 & 1,400 & 1,800 & 2,100 & 310.0 & 310.4 & 310.8 & \\
\hline 919 & $63+11$ & 30 & 1,400 & 1,800 & 2,100 & 311.8 & 312.2 & 312.5 & \\
\hline 920 & $66+08$ & 30 & 1,400 & 1,800 & 2,100 & 313.0 & 313.4 & 313.7 & \\
\hline 921 & $67+76$ & 30 & 1,400 & 1,800 & 2,100 & 313.8 & 314.1 & 314.4 & \\
\hline 922 & $69+14$ & 30 & 1,400 & 1,800 & 2,100 & 314.6 & 315.2 & 315.5 & \\
\hline 923 & $70+96$ & 30 & 1,400 & 1,800 & 2,100 & 318.5 & 319.0 & 319.4 & \\
\hline 924 & $72+76$ & 30 & 1,400 & 1,800 & 2,100 & 319.4 & 320.1 & 320.6 & \\
\hline 925 & $74+20$ & & & & & & & & Highway I-66 \\
\hline 926 & $75+56$ & 30 & 1,400 & 1,800 & 2,100 & 321.2 & 322.6 & 323.6 & \\
\hline 927 & $78+61$ & 30 & 1,400 & 1,800 & 2,100 & 321.5 & 322.8 & 323.7 & \\
\hline 928 & $82+46$ & 30 & 1,400 & 1,800 & 3,100 & 323.2 & 323.7 & 324.3 & \\
\hline 929 & $85+73$ & 30 & 1,400 & 1,800 & 2,100 & 325.6 & 325.9 & 326.0 & \\
\hline 930 & $89+01$ & 30 & 1,400 & 1,800 & 2,100 & 327.4 & 327.7 & 328.0 & \\
\hline 931 & $\begin{array}{l}91+12 \\
94+50\end{array}$ & 30 & 1,400 & 1,800 & 2,100 & 327.9 & 328.2 & 328.5 & Nutley Road \\
\hline
\end{tabular}

a Recurrence interval 
Accotink Creek Basin

Table 3: Water-surface profile data for Bear Branch

\begin{tabular}{|c|c|c|c|c|c|c|c|c|c|}
\hline $\begin{array}{l}\text { Section } \\
\text { number }\end{array}$ & $\begin{array}{c}\text { Base-line } \\
\text { Station } \\
\text { (feet) }\end{array}$ & $\begin{array}{l}\text { Impervi- } \\
\text { ousness } \\
\text { (per cent) }\end{array}$ & $\frac{\text { in cub }}{25-\text { year }^{a}}$ & $\begin{array}{l}\text { Discharge } \\
\text { c feet per } \\
50 \text {-year }\end{array}$ & $\frac{\text { second }}{100-\text { year }^{2}}$ & $25-$ year $^{2}$ & $\begin{array}{l}\text { Elevation, } \\
\text { in feet } \\
50 \text {-year }\end{array}$ & 100 -year & Remarks \\
\hline 806 & $13+92$ & 30 & 1,900 & 2,400 & 2,800 & 275.7 & 276.0 & 276.4 & \\
\hline 807 & $16+08$ & 30 & 1,900 & 2,400 & 2,800 & 276.6 & 276.8 & 277.1 & , \\
\hline 808 & $19+13$ & 30 & 1,900 & 2,400 & 2,800 & 277.8 & 278.1 & 278.2 & \\
\hline 809 & $21+06$ & 30 & 1,900 & 2,400 & 2,800 & 278.4 & 278.8 & 279.0 & \\
\hline 810 & $23+25$ & 30 & 1,900 & 2,400 & 2,800 & 279.2 & 279.5 & 279.8 & \\
\hline 811 & $24+98$ & 30 & 1,900 & 2,400 & 2,800 & 280.2 & 280.5 & 280.7 & \\
\hline 812 & $26+71$ & 30 & 1,900 & 2,400 & 2,800 & 281.0 & 281.2 & 281.5 & \\
\hline 813 & $27+30$ & & & & & & & & Arlington Blvd. (U.S 50) \\
\hline 814 & $28+06$ & 30 & 1,900 & 2,400 & 2,800 & 287.0 & 287.4 & 287.7 & \\
\hline 815 & $29+67$ & 30 & 1,900 & 2,400 & 2800 & 287.0 & 287.4 & 287.7 & \\
\hline 816 & $31+35$ & 30 & 1,900 & 2,400 & 2,800 & 287.0 & 287.4 & 287.7 & \\
\hline 817 & $33+57$ & 30 & 1,900 & 2,400 & 2,800 & 287.0 & 287.4 & 287.7 & \\
\hline 818 & $36+20$ & 30 & 1,900 & 2,400 & 2,800 & 287.3 & 287.7 & 288.1 & \\
\hline 819 & $39+29$ & 30 & 1,900 & 2,400 & 2,800 & 288.3 & 288.6 & 288.8 & \\
\hline 820 & $41+29$ & 30 & 1,900 & 2,400 & 2,800 & 289.1 & 289.3 & 289.5 & \\
\hline 821 & $43+22$ & 30 & 1,900 & 2,400 & 2,800 & 290.5 & 290.7 & 290.8 & \\
\hline 822 & $45+50$ & 30 & 1,900 & 2,400 & 2,800 & 291.8 & 292.0 & 292.2 & \\
\hline 823 & $47+33$ & 30 & 1,900 & 2,400 & 2,800 & 292.8 & 293.0 & 293.2 & \\
\hline 824 & $49+21$ & 30 & 1,900 & 2,400 & 2,800 & 293.9 & 294.2 & 294.4 & \\
\hline 825 & $51+02$ & 30 & 1,900 & 2,400 & 2,800 & 295.2 & 295.5 & 295.7 & \\
\hline 826 & $53+91$ & 30 & 1,900 & 2,400 & 2,800 & 297.0 & 297.4 & 297.6 & \\
\hline 827 & $56+37$ & 30 & 1,900 & 2,400 & 2,800 & 298.5 & 298.8 & 299.0 & \\
\hline 828 & $56+88$ & & & & & & & & Lee Hi hway (U.S. 29-211) \\
\hline 829 & $57+40$ & 30 & 1,900 & 2,400 & 2,800 & 302.0 & 302.5 & 302.8 & \\
\hline 830 & $59+19$ & 30 & 1,900 & 2,400 & 2800 & 302.1 & 302.6 & 302.9 & \\
\hline 831 & $60+48$ & 30 & 1,900 & 2,400 & 2,800 & 302.2 & 3028 & 303.0 & \\
\hline 832 & $61+99$ & 30 & 1,900 & 2,400 & 2,800 & 302.3 & 302.9 & 303.2 & \\
\hline 834 & $63+25$ & & & & & & & & Hunter Road \\
\hline 835 & $63+65$ & 30 & 1,900 & 2,400 & 2800 & 302.5 & 303.1 & 303.4 & \\
\hline 836 & $66+40$ & 30 & 1,900 & 2,400 & 2,800 & 303.0 & 303.5 & 303.8 & \\
\hline 837 & $68+58$ & 30 & 1,900 & 2,400 & 2,800 & 303.6 & 304.0 & 304.2 & \\
\hline 838 & $70+09$ & 30 & 1,900 & 2,400 & 2,800 & 304.4 & 304.7 & 304.9 & \\
\hline 839 & $72+60$ & 30 & 1,900 & 2,400 & 2,800 & 307.4 & 307.6 & 307.8 & \\
\hline 840 & $75+49$ & 30 & 1,900 & 2,400 & 2,800 & 308.2 & 308.6 & 308.7 & \\
\hline
\end{tabular}


Accotink Creek Basin

Table 4: Water-surface profile data for Long Branch near Vienna

\begin{tabular}{lr}
$\begin{array}{lr}\text { Section } \\
\text { number }\end{array}$ & $\begin{array}{r}\text { Base- }-1 \text { in } \\
\text { (feet) }\end{array}$ \\
\hline & \\
703 & $12+48$ \\
704 & $15+62$ \\
705 & $18+19$ \\
706 & $20+92$ \\
707 & $23+48$ \\
708 & $25+80$ \\
709 & $28+72$ \\
710 & $31+00$ \\
711 & $32+40$ \\
712 & $34+15$ \\
713 & $35+35$ \\
714 & $37+70$ \\
715 & $40+55$ \\
716 & $42+34$ \\
717 & $44+15$ \\
718 & $46+41$ \\
719 & $47+10$ \\
720 & $47+92$ \\
721 & $49+64$ \\
722 & $51+58$ \\
723 & $54+27$ \\
724 & $56+49$ \\
725 & $59+23$ \\
726 & $61+20$ \\
727 & $63+09$ \\
728 & $65+81$ \\
729 & $68+64$ \\
730 & $70+58$ \\
731 & $72+03$ \\
732 & $73+87$
\end{tabular}

Discharge,

Elevation,

ousness

in cubic feet per second

$\frac{\text { in feet }}{25 \text {-year }}$

$100-$ yeara $^{2}$

Remarks (per cent)

25 -yeara

50 -year ${ }^{a} 10$


Accotink Creek Basin

Table 4: Water-surface profile data for Long Branch near Vienna - Continued

\begin{tabular}{|c|c|c|c|c|c|c|c|c|c|}
\hline \multirow{2}{*}{$\begin{array}{l}\text { Section } \\
\text { number }\end{array}$} & \multirow{2}{*}{$\begin{array}{c}\text { Base-line } \\
\text { Station } \\
\text { (feet) }\end{array}$} & \multirow{2}{*}{$\begin{array}{l}\text { Impervi- } \\
\text { ousness } \\
\text { (per cent) }\end{array}$} & \multicolumn{3}{|c|}{$\begin{array}{c}\text { Discharge, } \\
\text { in cubic feet per second } \\
\end{array}$} & \multicolumn{3}{|c|}{$\begin{array}{c}\text { Elevation, } \\
\text { in feet } \\
\end{array}$} & \multirow[t]{2}{*}{ Remarks } \\
\hline & & & 25-yeara & 50 -yeara & $100-$ yeara $^{a}$ & 25 -yeara & 50-yeara & 100 -yeara & \\
\hline 733 & $75+49$ & 40 & 1,500 & 1,800 & 2,200 & 304.1 & 304.4 & 305.2 & \\
\hline 734 & $77+29$ & 40 & 1,500 & 1,800 & 2,200 & 305.6 & 305.9 & 306.2 & . \\
\hline 735 & $77+39$ & & & & & & & & Private Road \\
\hline 737 & $77+95$ & 40 & 1,500 & 1,800 & 2,200 & 306.0 & 306.2 & 306.6 & \\
\hline 738 & $78+42$ & & & & & & & & Lee Highway(U.S. 29-211) \\
\hline 739 & $79+30$ & 40 & 1,500 & 1,800 & 2,200 & 309.8 & 311.1 & 312.8 & \\
\hline 740 & $80+21$ & 40 & 1,500 & 1,800 & 2,200 & 309.8 & 311.2 & 312.8 & \\
\hline 741 & $81+65$ & 40 & 1,500 & 1,800 & 2,200 & 309.8 & 311.2 & 312.8 & \\
\hline 742 & $81+90$ & & & & & & & & Old Lee Highway \\
\hline 743 & $82+18$ & 40 & 1,500 & 1,800 & 2,200 & 311.7 & 312.3 & 313.0 & \\
\hline 744 & $84+25$ & 40 & 1,500 & 1,800 & 2,200 & 311.7 & 312.3 & 313.0 & \\
\hline 745 & $85+59$ & 40 & 1,500 & 1,800 & 2,200 & 311.8 & 312.4 & 313.0 & \\
\hline 746 & $87+49$ & 40 & 1,500 & 1,800 & 2,200 & 311.8 & 312.4 & 313.1 & \\
\hline 747 & $89+62$ & 40 & 1,500 & 1,800 & 2,200 & 312.4 & 313.0 & 313.5 & \\
\hline 748 & $91+62$ & 40 & 1,500 & 1,800 & 2,200 & 313.5 & 3138 & 314.4 & \\
\hline 749 & $92+05$ & & & & & & & & Hilltop Road \\
\hline
\end{tabular}


Accotink Creek Basin

Table 5: Water-surface profile data for Crook Branch

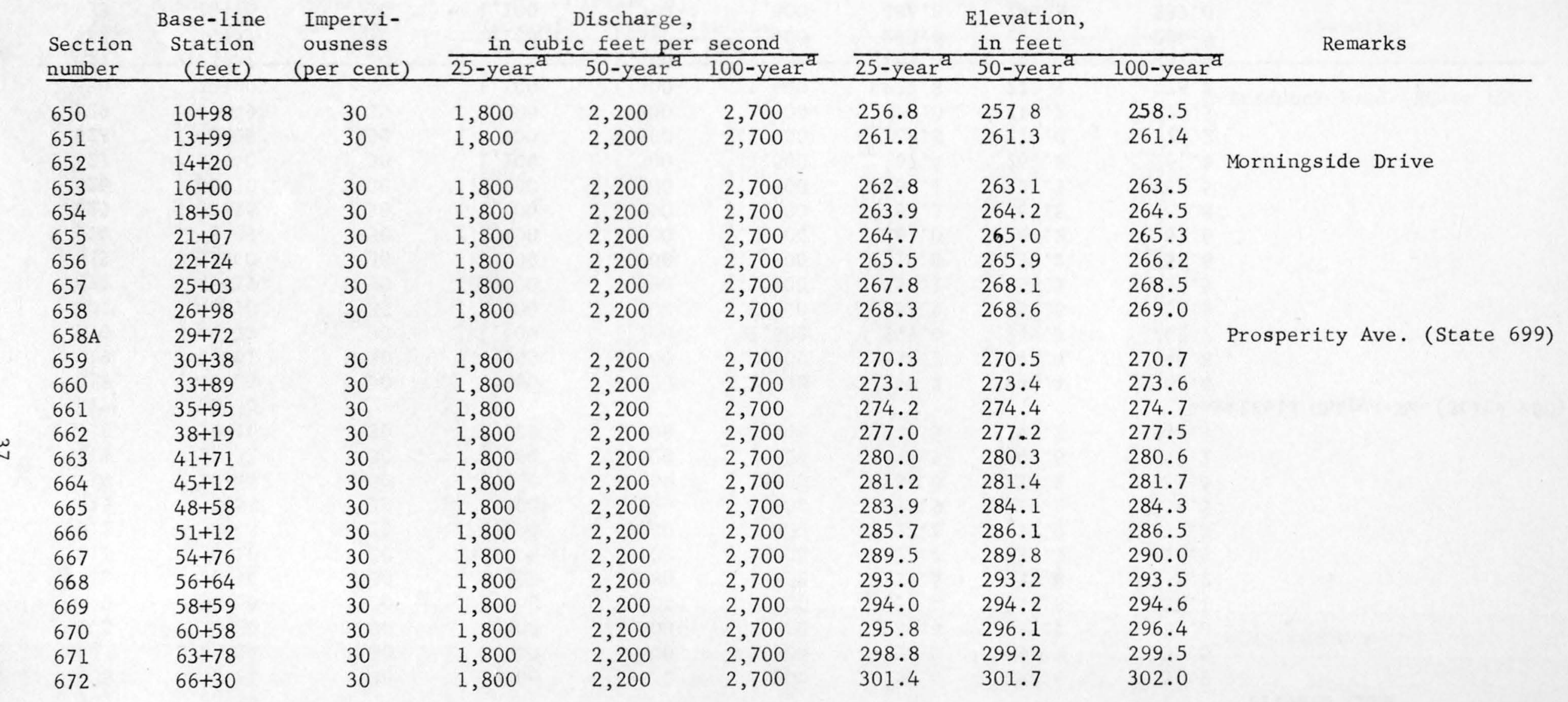


Accotink Creek Basin

Table 6: Water-surface profile data for Turkey Run

Base-line Impervi Discharge, Elevation,

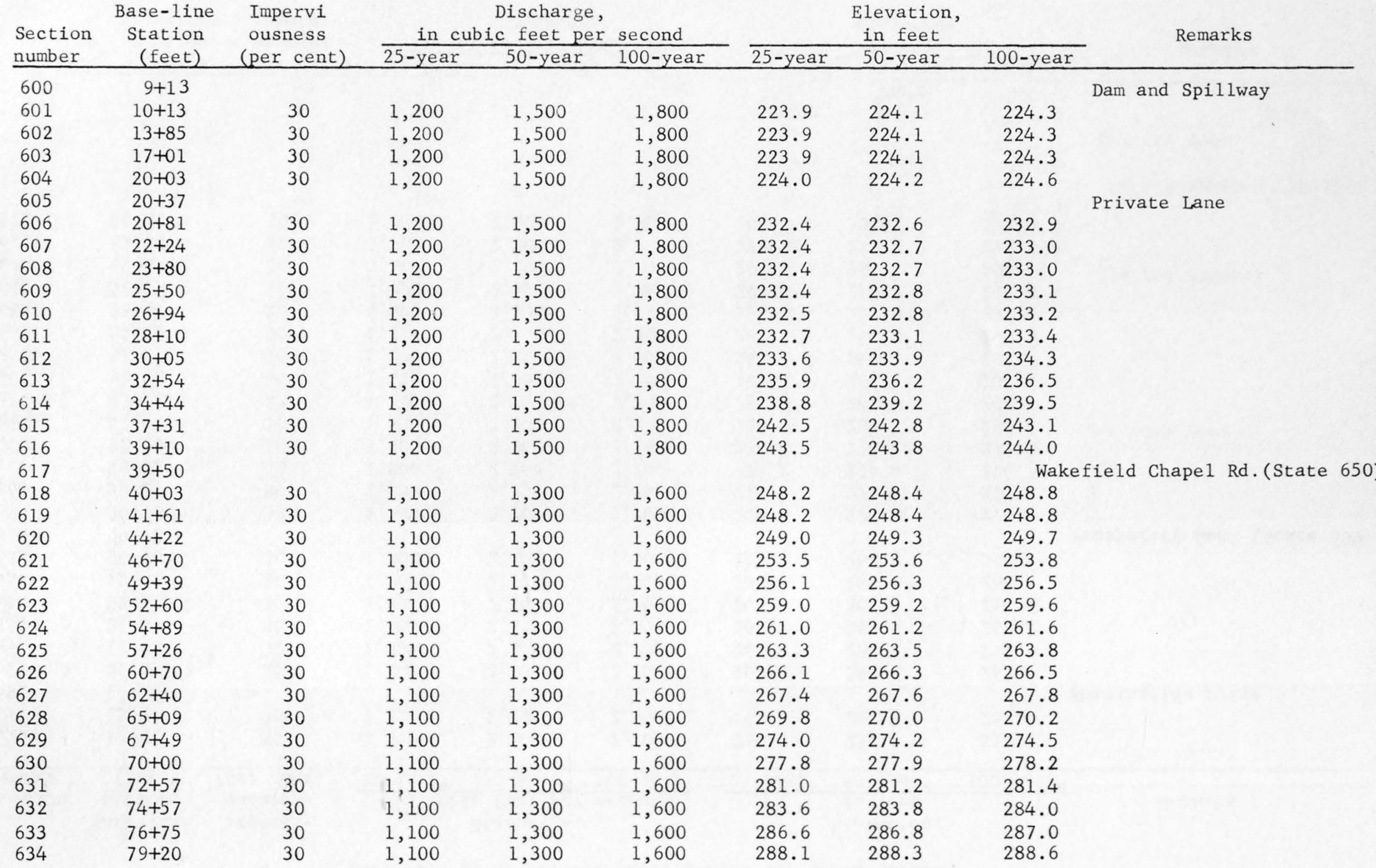

a Recurrence interval 
Accotink Creek Basin

Table 7: Water-surface profile data for Long Branch near Annandale

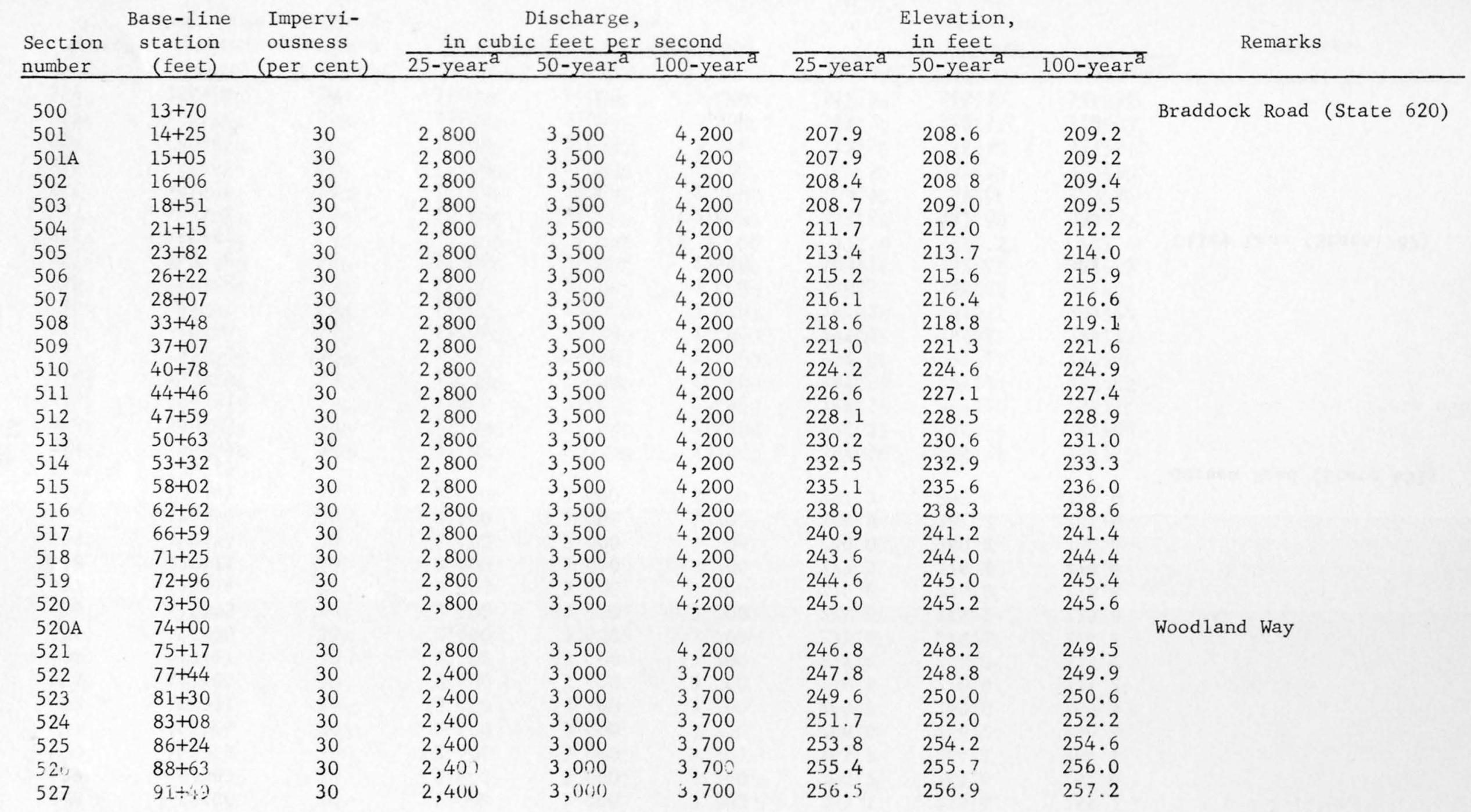

\footnotetext{
a Recurrence interval
} 
Accotink Creek Basin

Table 7: Water-surface profile data for Long Branch near Annandale - Continued

\begin{tabular}{|c|c|c|c|c|c|c|c|c|c|c|}
\hline \multirow{2}{*}{$\begin{array}{l}\text { Section } \\
\text { number }\end{array}$} & \multirow{2}{*}{$\begin{array}{c}\text { Base-line } \\
\text { Station } \\
\text { (feet) }\end{array}$} & \multirow{2}{*}{$\begin{array}{l}\text { Impervi- } \\
\text { ousness } \\
\text { (per cent) }\end{array}$} & \multicolumn{3}{|c|}{$\begin{array}{l}\text { Discharge, } \\
\text { in cubic feet per second }\end{array}$} & \multicolumn{3}{|c|}{$\begin{array}{c}\text { Elevation, } \\
\text { in feet }\end{array}$} & \multirow{2}{*}{\multicolumn{2}{|c|}{ Remarks }} \\
\hline & & & 25 -year ${ }^{a}$ & 50 -year & 100 -year ${ }^{a}$ & 25 -yeara & $50-$ year $^{a}$ & $100-$ year $^{a}$ & & \\
\hline 528 & $95+06$ & 30 & 2,400 & 3,000 & 3,700 & 259.1 & 259.4 & 259.7 & & \\
\hline 529 & $97+89$ & 30 & 2,400 & 3,000 & 3,700 & 260.7 & 261.0 & 261.4 & & \\
\hline 530 & $102+29$ & 30 & 2,400 & 3,000 & 3,700 & 263.7 & 264.1 & 264.4 & & · \\
\hline 531 & $105+26$ & 30 & 2,400 & 3,000 & 3,700 & 266.0 & 266.2 & 266.6 & & \\
\hline 532 & $108+77$ & 30 & 2,400 & 3,000 & 3,700 & 268.7 & 269.0 & 269.3 & & \\
\hline 533 & $111+03$ & 30 & 2,400 & 3,000 & 3,700 & 270.6 & 270.9 & 271.2 & & \\
\hline 534 & $113+11$ & 30 & 2,400 & 3,000 & 3,700 & 272.4 & 272.7 & 273.0 & & \\
\hline 535 & $114+70$ & 30 & 2,400 & 3,000 & 3,700 & 273.8 & 274.1 & 274.5 & & \\
\hline 536 & $115+95$ & 30 & 2,400 & 3,000 & 3,700 & 274.8 & 275.2 & 275.6 & & \\
\hline 537 & $118+14$ & 30 & 2,400 & 3,000 & 3,700 & 2758 & 276.2 & 276.6 & & \\
\hline 538 & $120+11$ & 30 & 2,400 & 3,000 & 3,700 & 277.5 & 278.1 & 278.6 & & \\
\hline 539 & $122+56$ & 30 & 2,400 & 3,000 & 3,700 & 280.0 & 280.2 & 280.5 & & \\
\hline 540 & $124+50$ & 30 & 2,400 & 3,000 & 3,700 & 280.9 & 281.2 & 281.6 & & \\
\hline 541 & $126+41$ & 30 & 2,400 & 3,000 & 3,700 & 282.3 & 282.6 & 283.0 & & \\
\hline 542 & $126+75$ & & & & & & & & Guinea & Road (State 651) \\
\hline 543 & $127+07$ & 30 & 2,400 & 3,000 & 3,700 & 283.9 & 284.2 & 284.5 & & \\
\hline 544 & $129+00$ & 30 & 1,600 & 2,000 & 2,400 & 284.2 & 284.5 & 284.8 & & \\
\hline 545 & $130+83$ & 30 & 1,600 & 2,000 & 2,400 & 285.1 & 285.2 & 285.5 & & \\
\hline 546 & $133+69$ & 30 & 1,600 & 2,000 & 2,400 & 287.5 & 287.7 & 287.8 & & \\
\hline 547 & $137+09$ & 30 & 1,600 & 2,000 & 2,400 & 290.9 & 291.1 & 291.4 & & \\
\hline 548 & $140+60$ & 30 & 1,600 & 2,000 & 2,400 & 295.2 & 295.5 & 295.7 & & \\
\hline 549 & $143+75$ & 30 & 1,600 & 2,000 & 2,400 & 297.2 & 297.5 & 297.7 & & \\
\hline 550 & $147+68$ & 30 & 1,600 & 2,000 & 2,400 & 300.5 & 300.8 & 301.0 & & \\
\hline 551 & $150+38$ & 30 & 1,600 & 2,000 & 2,400 & 303.1 & 303.4 & 303.6 & & \\
\hline 552 & $150+62$ & & & & & & & & 011ey & Lane (State 787) \\
\hline 553 & $150+94$ & 30 & 1,600 & 2,000 & 2,400 & 306.6 & 307.0 & 307.3 & & \\
\hline 554 & $153+22$ & 30 & 1,600 & 2,000 & 2,400 & 306.7 & 307.0 & 307.4 & & \\
\hline 555 & $155+51$ & 30 & 1,600 & 2,000 & 2,400 & 307.1 & 307.5 & 307.8 & & \\
\hline 556 & $158+93$ & 30 & 1,600 & 2,000 & 2,400 & 311.3 & 311.6 & 311.8 & & \\
\hline 557 & $161+90$ & 30 & 1,600 & 2,000 & 2,400 & 3132 & 313.5 & 313.7 & & \\
\hline 558 & $164+84$ & 30 & 1,600 & 2,000 & 2,400 & 314.8 & 315.1 & 315.3 & & \\
\hline
\end{tabular}


Accotink Creek Basin

Table 7: Water-surface profile data for Long Branch near Annandale - Continued

\begin{tabular}{|c|c|c|c|c|c|c|c|c|c|}
\hline $\begin{array}{l}\text { Section } \\
\text { number }\end{array}$ & $\begin{array}{c}\text { Base-1ine } \\
\text { station } \\
\text { (feet) }\end{array}$ & $\begin{array}{l}\text { Impervi- } \\
\text { ousness } \\
\text { (per cent) }\end{array}$ & $\frac{\text { in cub }}{25-\text { year }^{\mathrm{a}}}$ & $\begin{array}{l}\text { Discharge, } \\
\text { c feet per } \\
50 \text {-year }\end{array}$ & $\frac{\text { second }}{100-\text { year }^{a}}$ & 25 -year ${ }^{\mathrm{a}}$ & $\begin{array}{l}\text { Elevation, } \\
\text { in feet } \\
50 \text {-year }\end{array}$ & 100 -year ${ }^{a}$ & Remarks \\
\hline 559 & $167+80$ & 30 & 1,600 & 2,000 & 2,400 & 317.6 & 317.9 & 318.2 & \\
\hline 560 & $171+30$ & 30 & 1,600 & 2,000 & 2,400 & 320.7 & 321.1 & 321.3 & \\
\hline 561 & $174+29$ & 30 & 1,600 & 2,000 & 2,400 & 322.6 & 322.9 & 323.1 & \\
\hline 562 & $177+09$ & 30 & 1,600 & 2,000 & 2,400 & 326.0 & 326.3 & 326.5 & \\
\hline 563 & $179+42$ & 30 & 1,600 & 2,000 & 2,400 & 330.0 & 330.4 & 330.6 & \\
\hline 564 & $181+69$ & 30 & 1,600 & 2,000 & 2,400 & 331.6 & 332.0 & 332.3 & \\
\hline 565 & $183+59$ & 30 & 1,600 & 2,000 & 2,400 & 332.8 & 333.2 & 333.6 & \\
\hline 566 & $185+60$ & 30 & 1,600 & 2,000 & 2,400 & 335.8 & 336.1 & 336.3 & \\
\hline 567 & $187+95$ & 30 & 1,600 & 2,000 & 2,400 & 337.8 & 338.2 & 338.5 & \\
\hline 568 & $189+65$ & 30 & 1,600 & 2,000 & 2,400 & 338.8 & 339.1 & 339.4 & \\
\hline 569 & $191+95$ & 30 & 1,600 & 2,000 & 2,400 & 340.5 & 340.9 & 341.2 & \\
\hline 570 & $194+12$ & 30 & 1,600 & 2,000 & 2,400 & 341.4 & 341.7 & 342.0 & \\
\hline 571 & $196+08$ & 30 & 1,600 & 2,000 & 2,400 & 343.9 & 344.1 & 344.2 & \\
\hline 572 & $198+25$ & 30 & 1,600 & 2,000 & 2,400 & 346.6 & 347.0 & 347.3 & \\
\hline 573 & $201+49$ & 30 & 1,600 & 2,000 & 2,400 & 349.2 & 349.6 & 349.9 & \\
\hline 574 & $203+74$ & 30 & 1,600 & 2,000 & 2,400 & 350.9 & 351.2 & 351.5 & \\
\hline
\end{tabular}

a Recurrence interva1 
Accotink Creek Basin

Table 8: Water-surface profile data for Long Branch near Newington

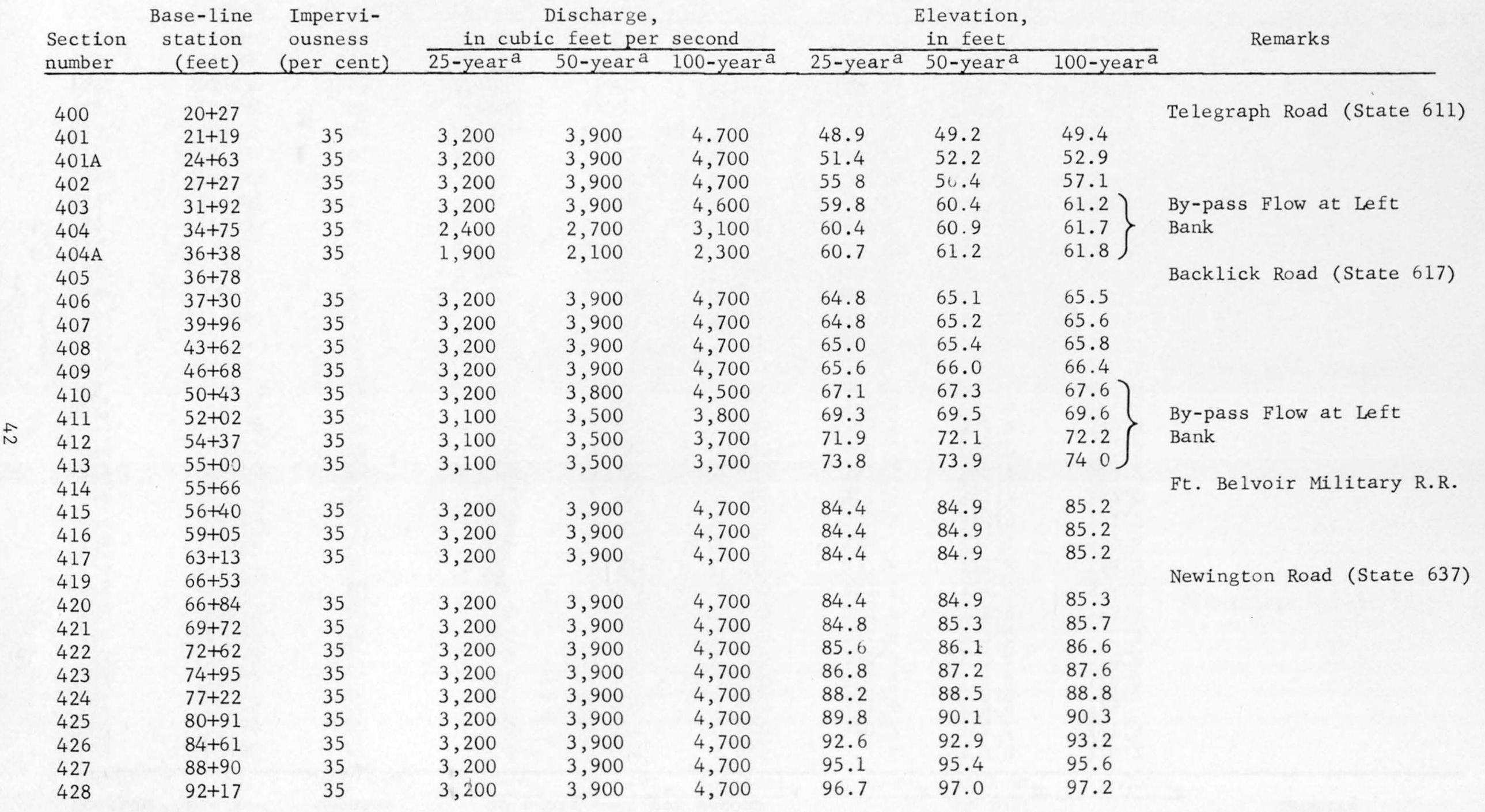

\footnotetext{
${ }^{a}$ Recurrence interval
} 
Accotink Creek Basin

Table 8: Water-surface profile data for Long Branch near Newington - Continued

Base-line Impervi- Discharge, Elevation,

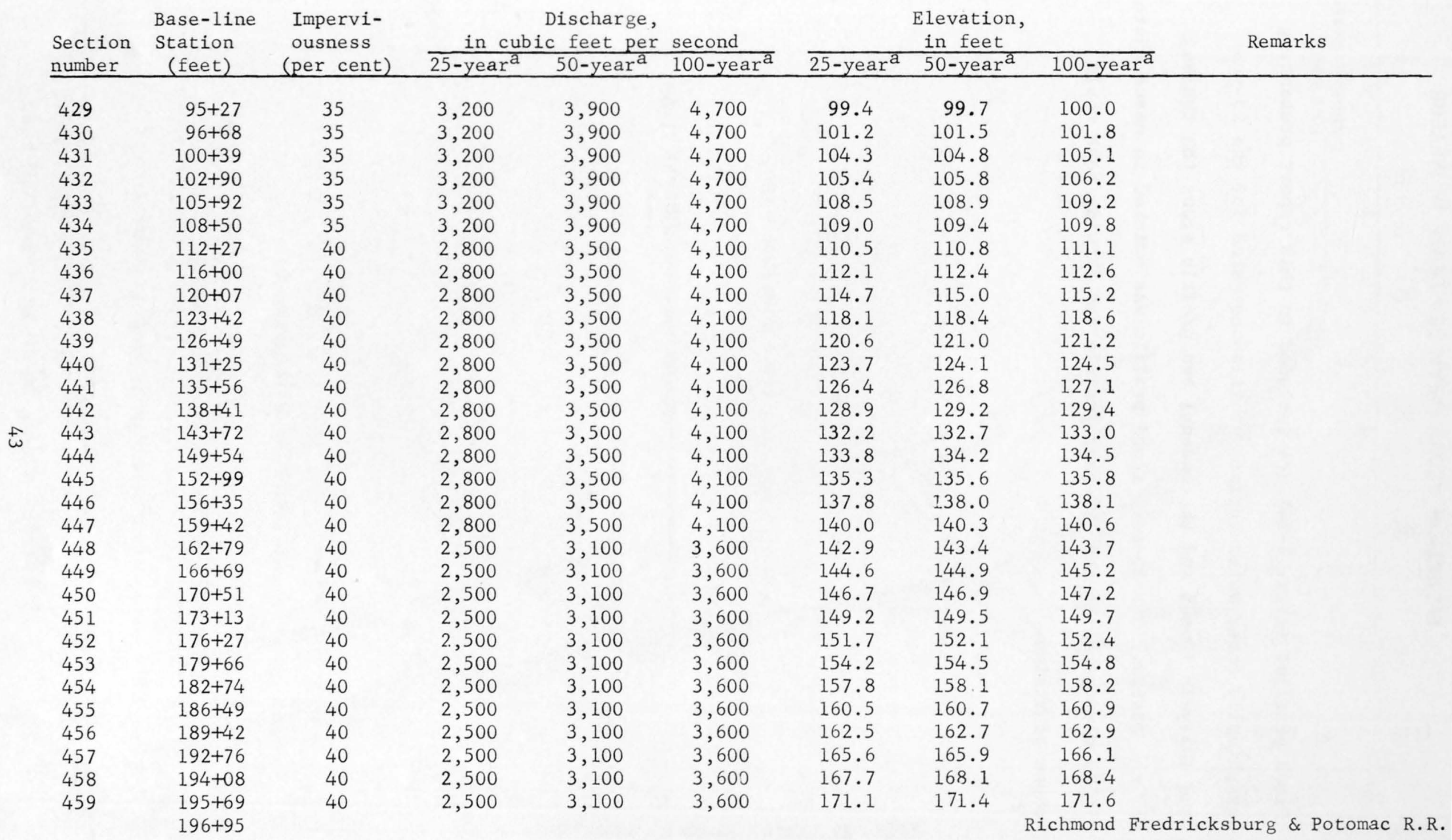

a Recurrence interval 
Flood profiles (figs. 3-14) are included in this report presenting graphically those water-surface profiles computed for the 25-year and 100-year floods and the channel bed profile along the thalweg of the stream. The 25-year flood profile was omitted in cases where it is very close to the 100-year profile and showing both would cause confusion. 

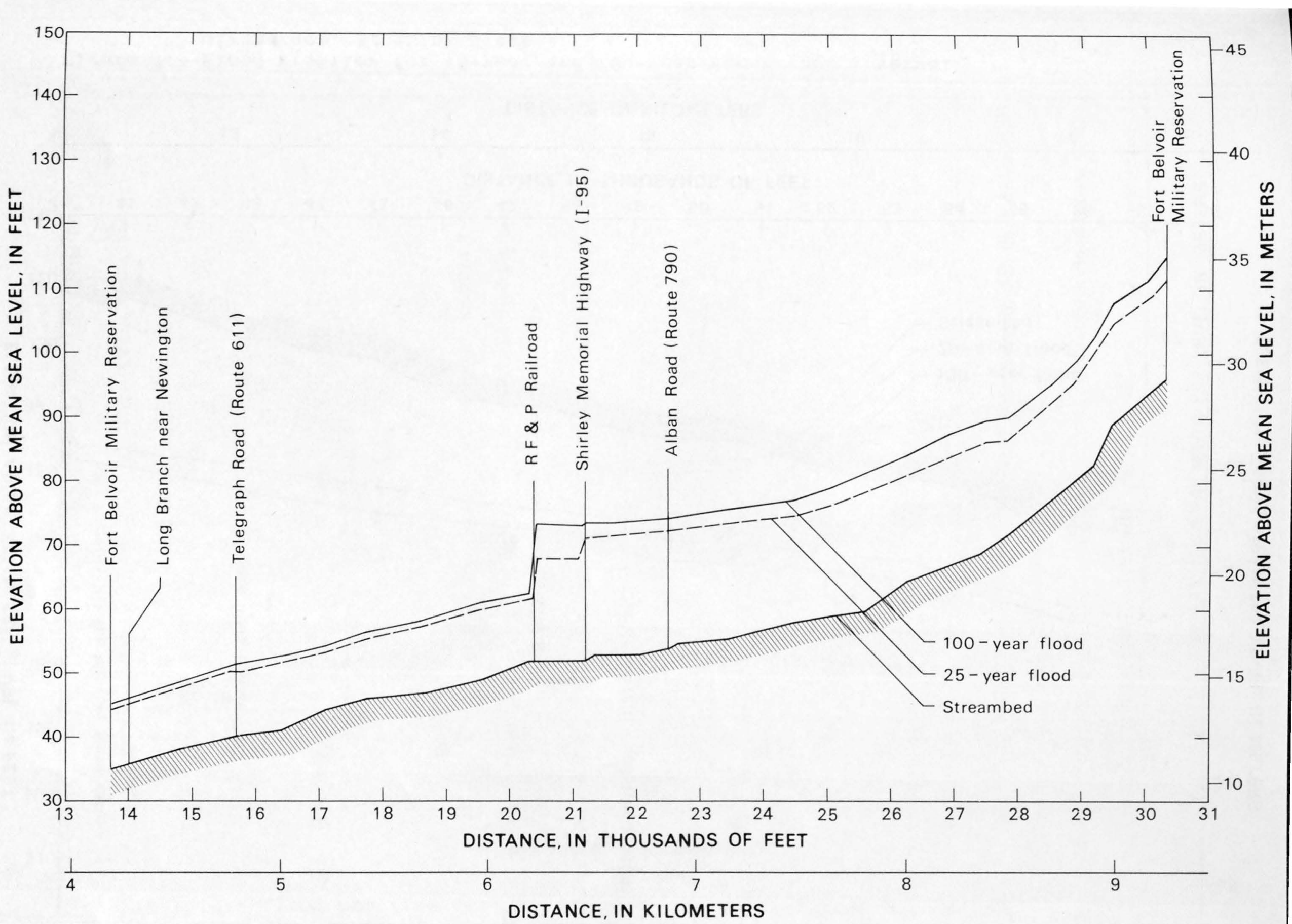

Figure 3.--Flood profiles for 25-year and 100-year recurrence interval discharges, Accotink Creek 


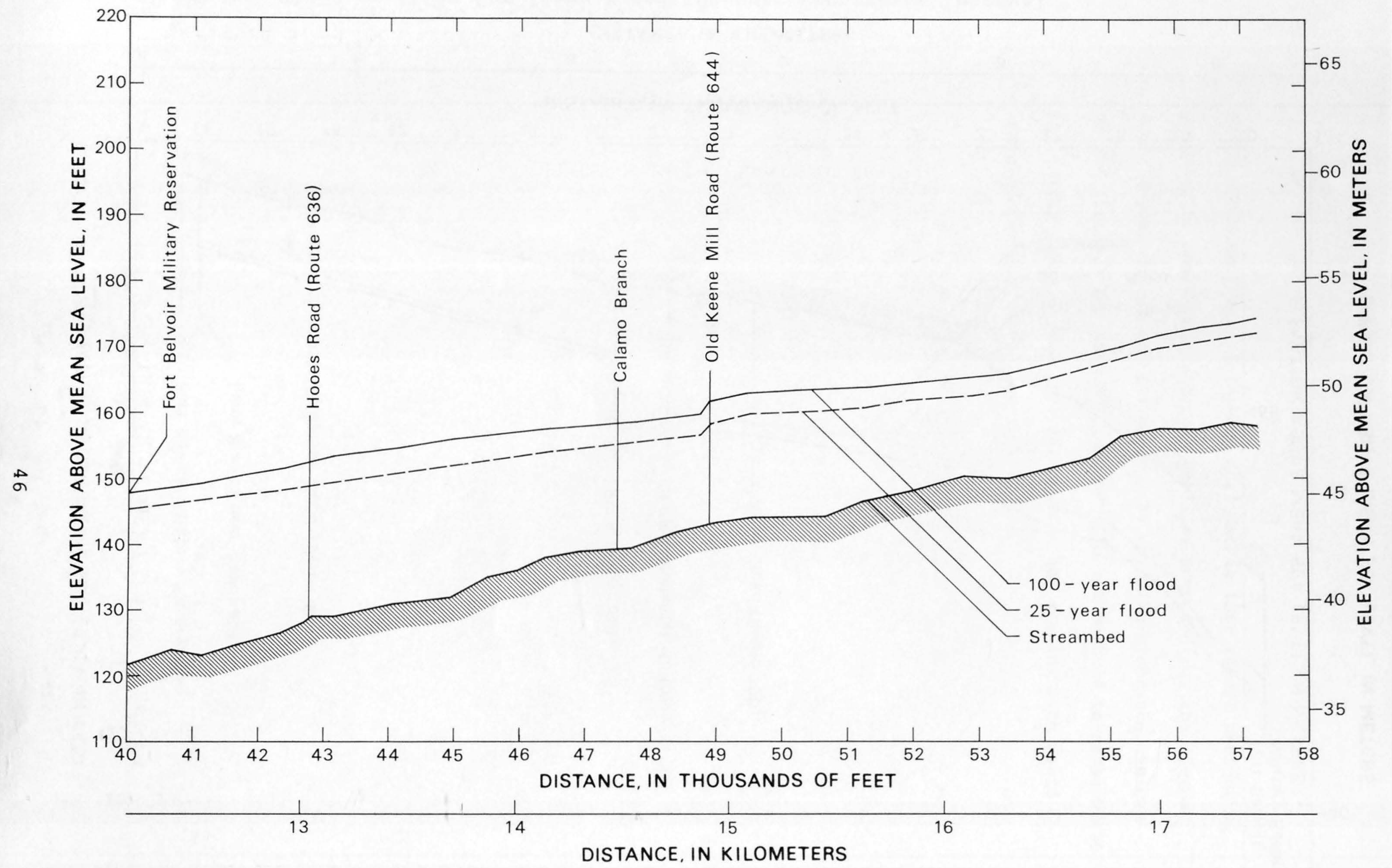

Figure 4.--Flood profiles for 25-year and 100-year recurrence interval discharges, Accotink Creek 


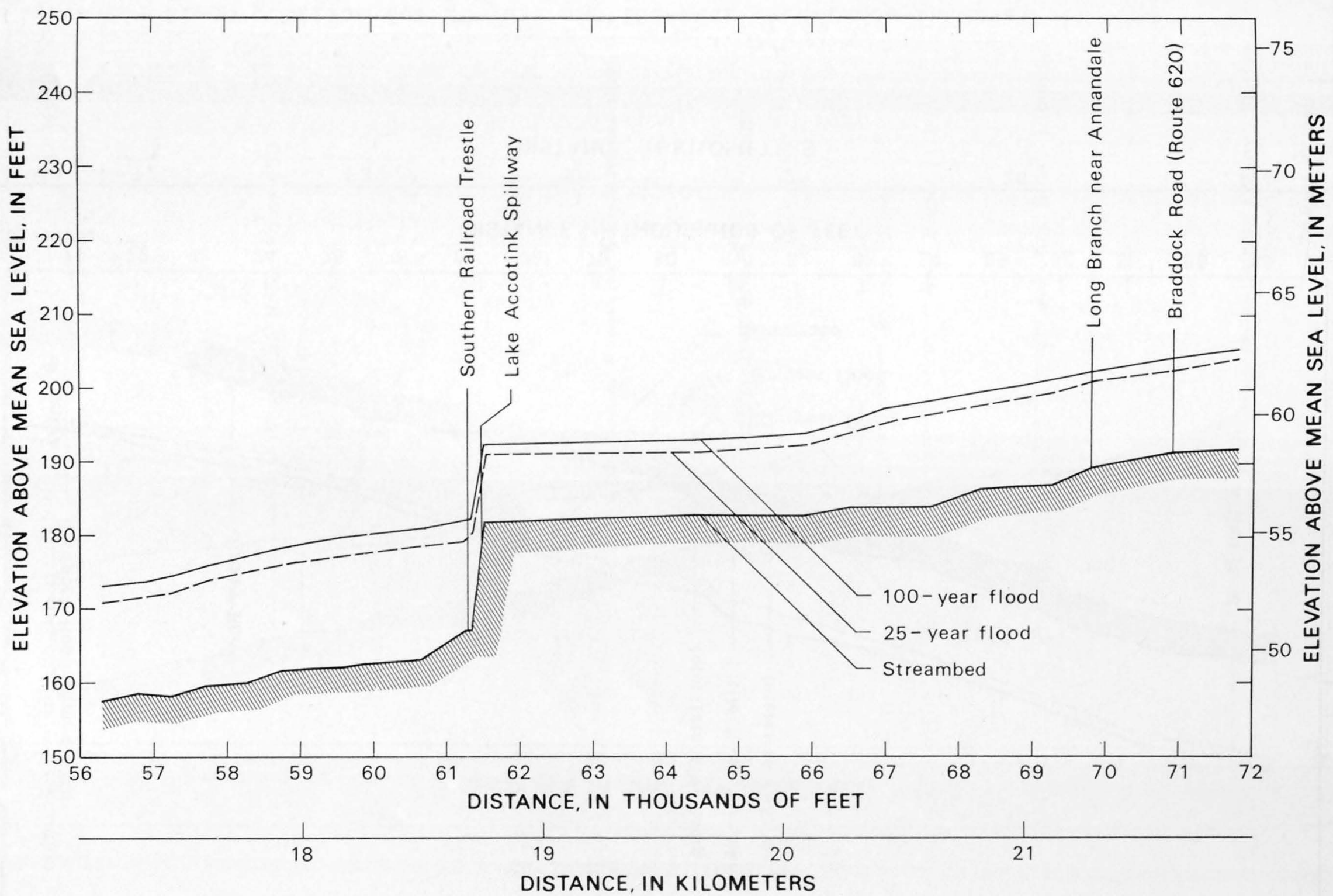

Figure 5.--Flood profiles for 25-year and 100-year recurrence interval discharges, Accotink Creek 


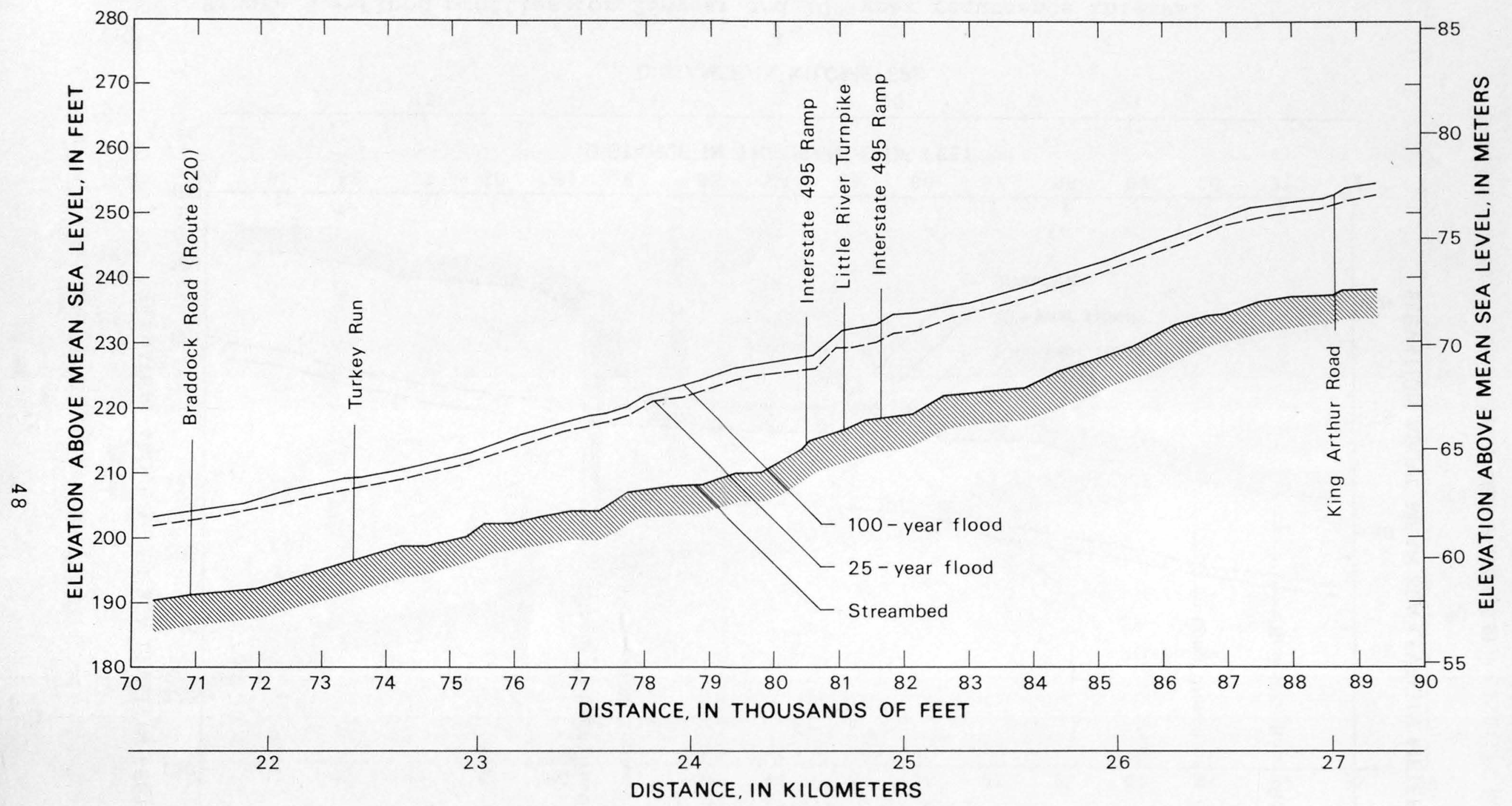

Figure 6.--Flood profiles for 25-year and 100-year recurrence interval discharges, Accotink Creek 


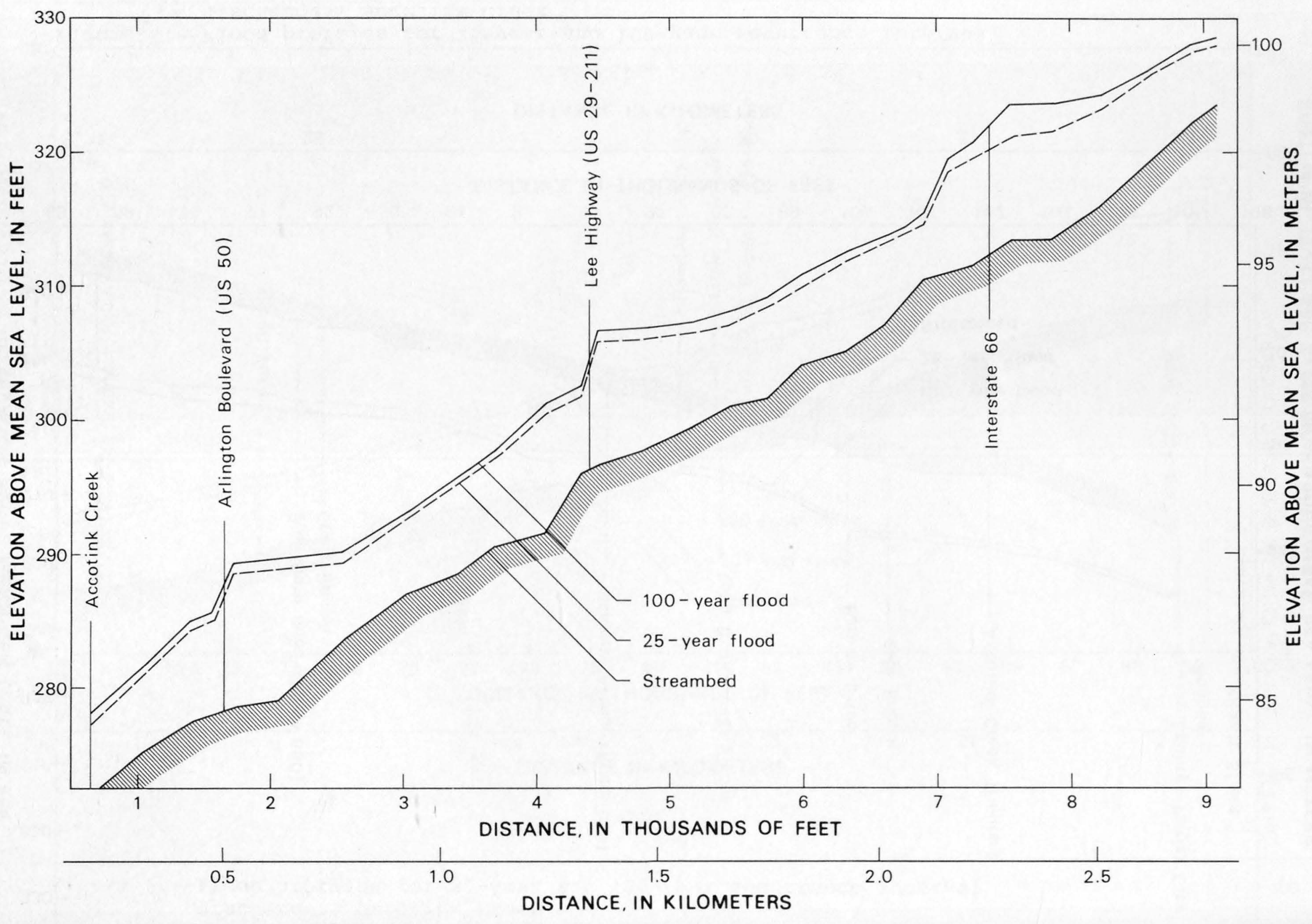

Figure 8.--Flood profiles for 25-year and 100-year recurrence interval discharges, Accotink Creek Tributary 


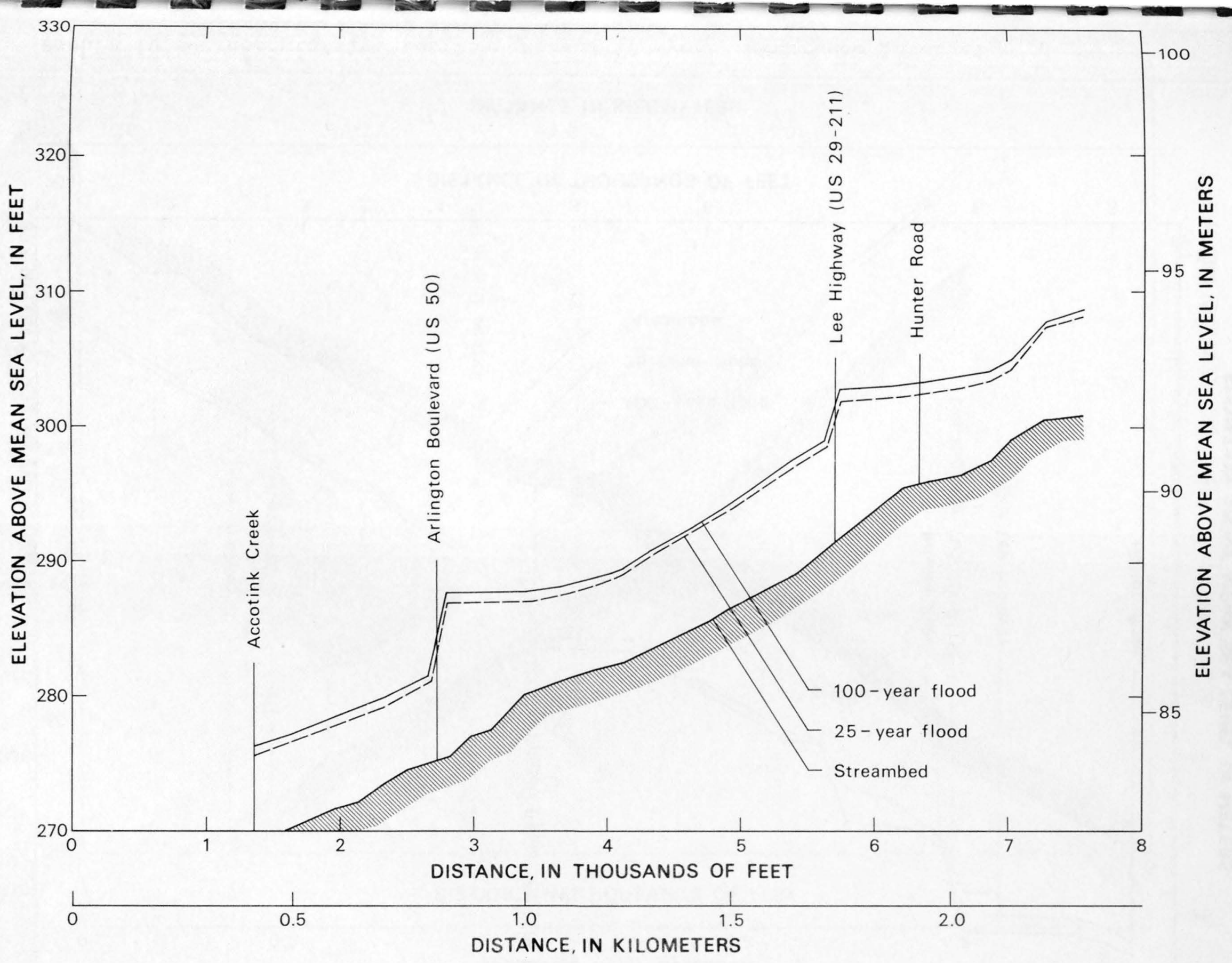

Figure 9.--Flood profiles for 25-year and 100-year recurrence interval discharges, Bear Branch 


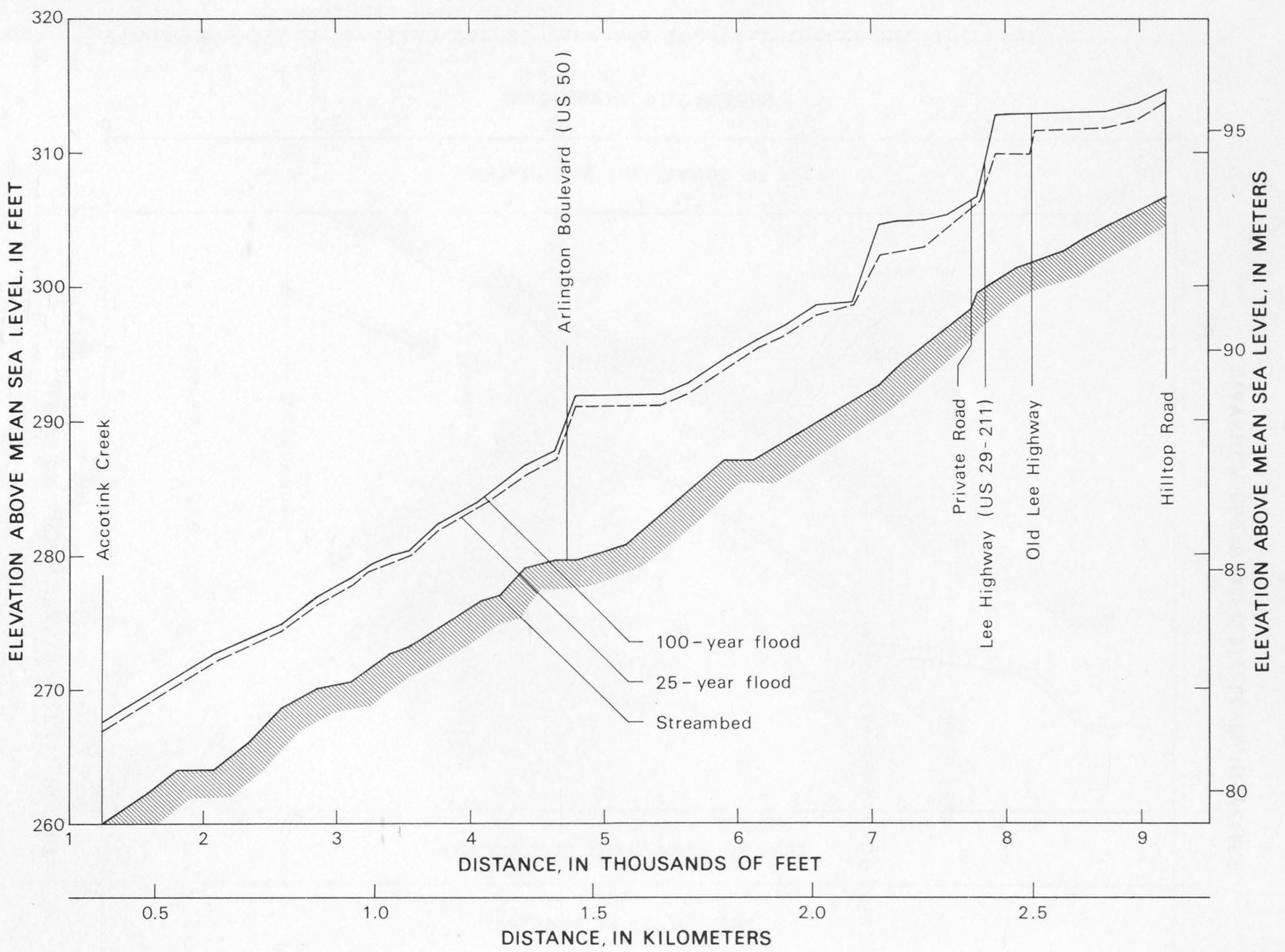

Figure 10.--Flood profiles for 25-year and 100-year recurrence interval 


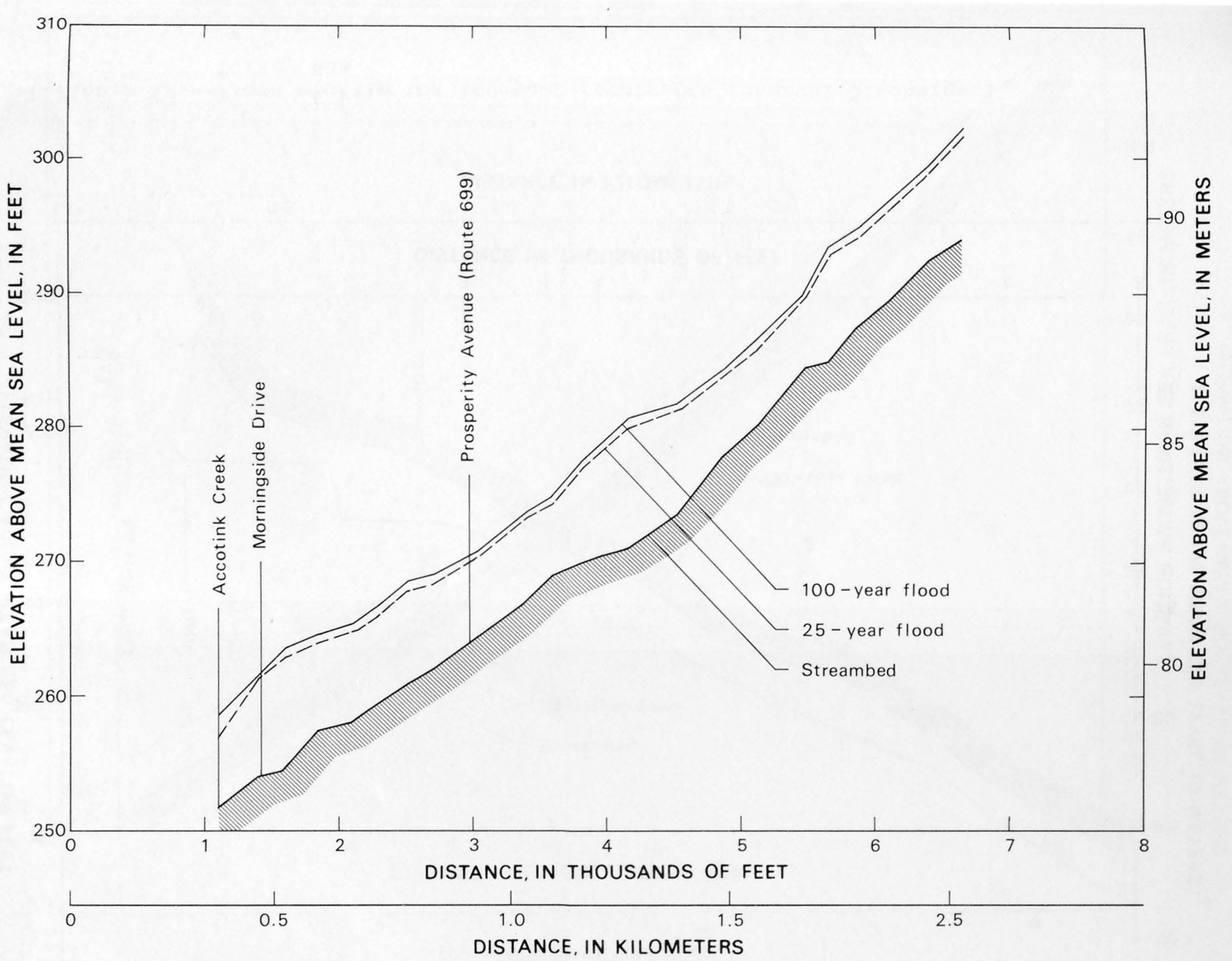

Figure 11.--Flood profiles for 25-year and 100-year recurrence interval discharges, Crook Branch 


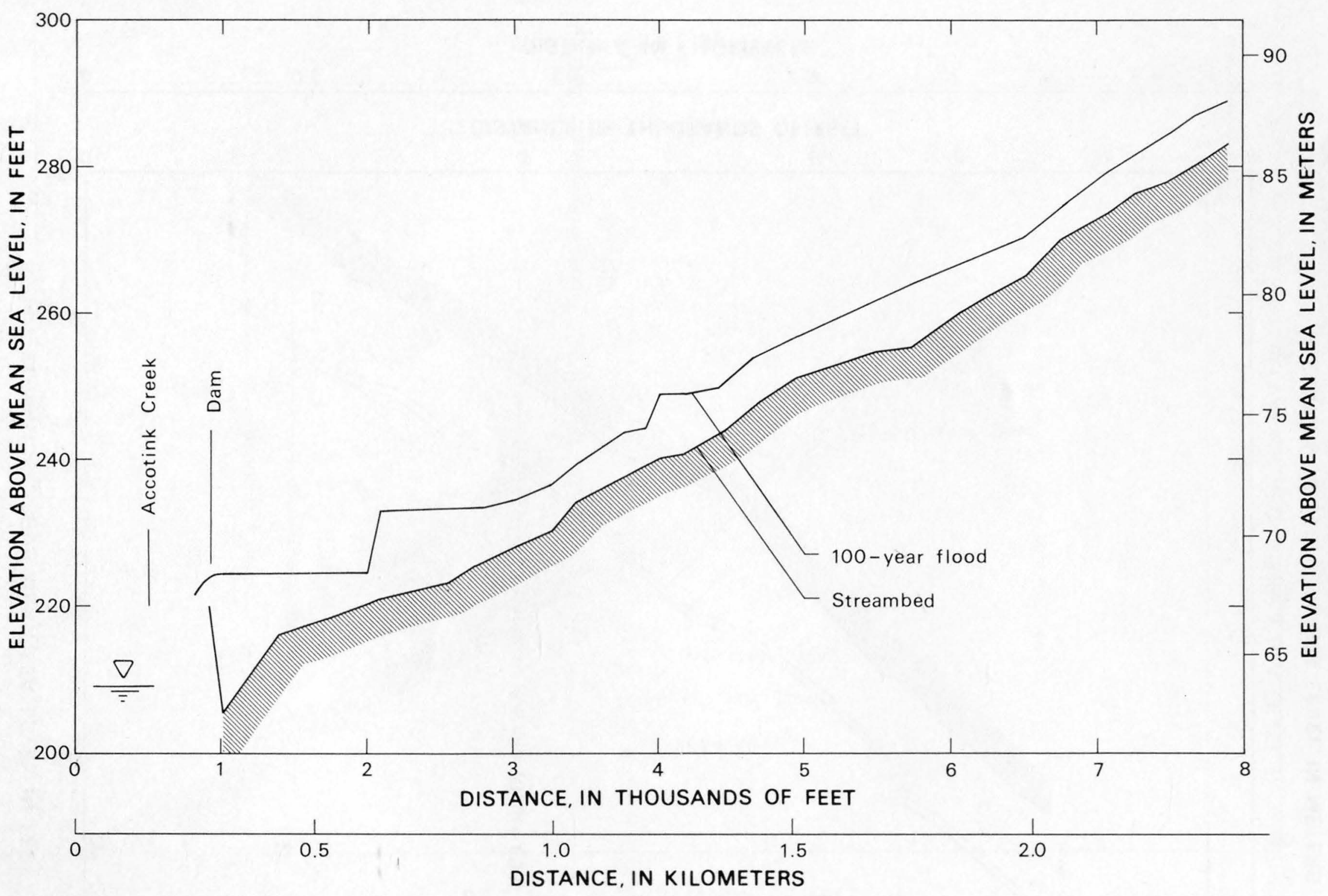

Figure 12.--Flood profile for 100-year recurrence interval discharges, Turkey Run 


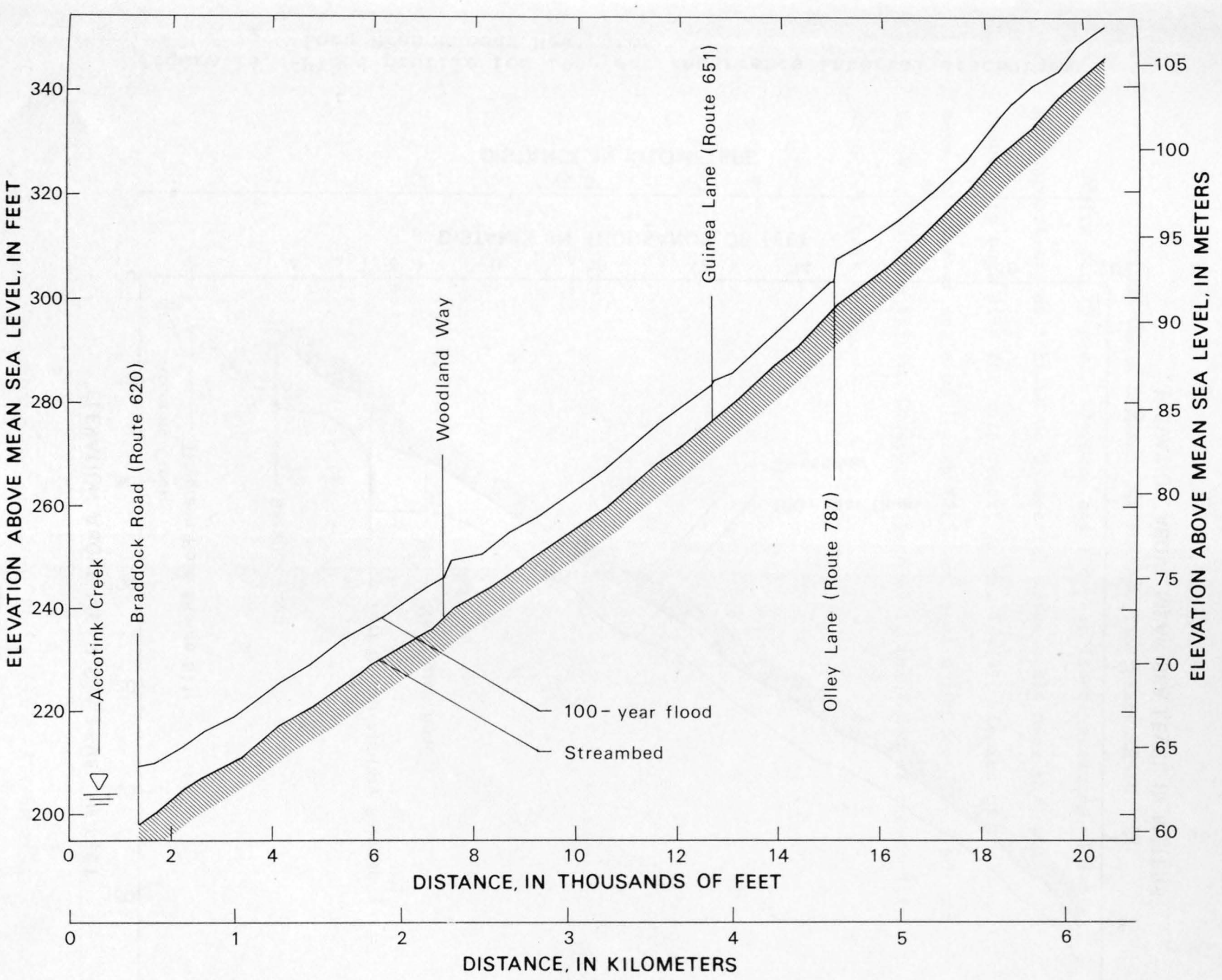

Figure 13.--Flood profile for 100-year recurrence interval discharges, Long Branch near Annandale 

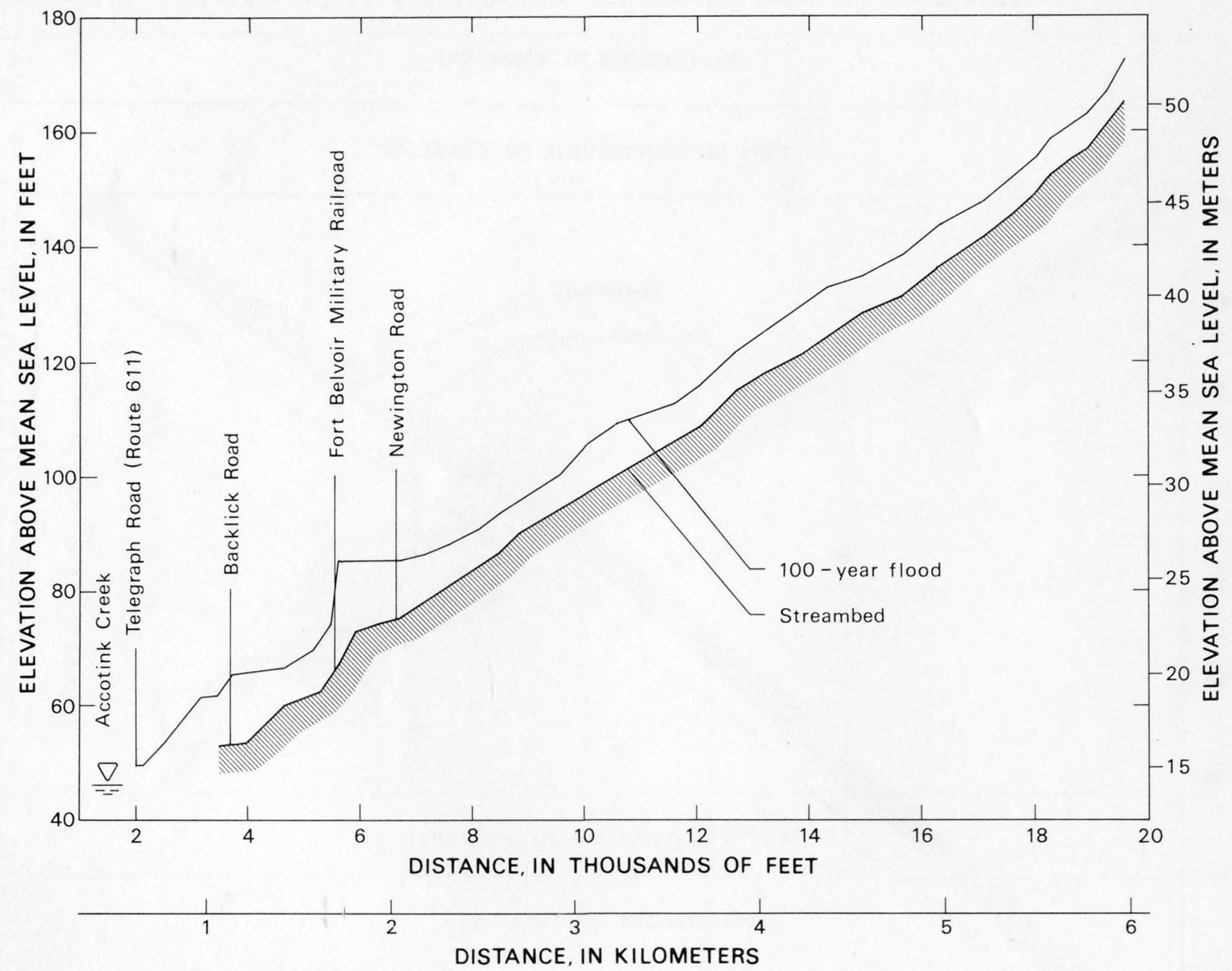

Figure 14.--Flood profile for 100-year recurrence interval discharges, Long Branch near Newington 
FLOOD-PLAIN DELINEATION FOR ACCOTINK CREEK BASIN

Limits for 25-year, 50-year, and 100-year floods assuming ultimate development are delineated on 59 special large scale maps that were reduced to page size for this report (Figs. 16-74). Copies of the maps at original scale of 1 inch $(2.54 \mathrm{~cm})$ equals 100 feet $(30.5 \mathrm{~m})$ can be obtained from Fairfax County. Figure 15 is the index of sheets for Accotink Creek basin. 


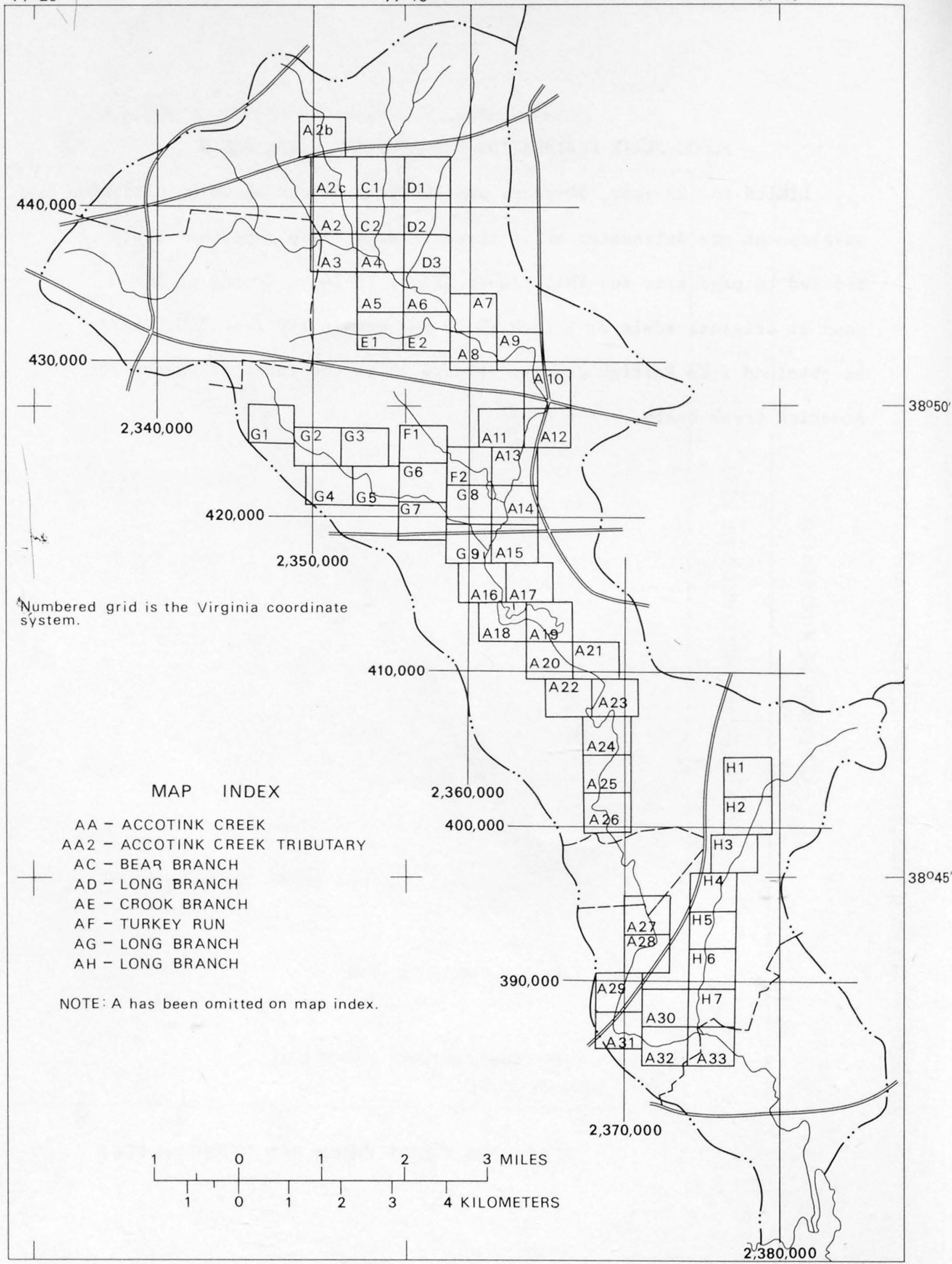

Figure 15.--Index of flood maps 


\section{DELINEATION OF FLOOD AREAS}

ACCOTINK CREEK BASIN

Flood plain delineation by U.S. Geological Survey in cooperation with the County of Fairfax as a part of a study of urbanization effects upon flood discharges.

Topography from aerial photographs taken 1960 \& 1963 250-foot grid based on Virginia coordinate system north zone.

Maps compiled by photogrammetric methods. Control and photogrammetric surveys are in accordance with Nationa1 Map Accuracy Standards.

\section{SYMBOLS}

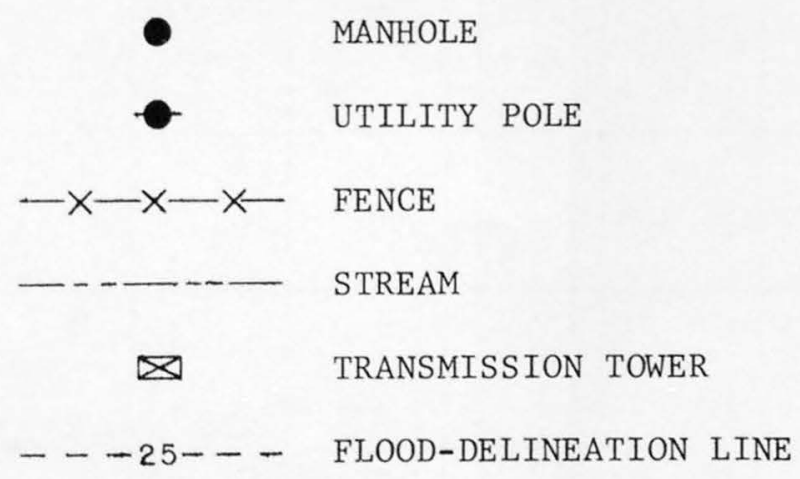




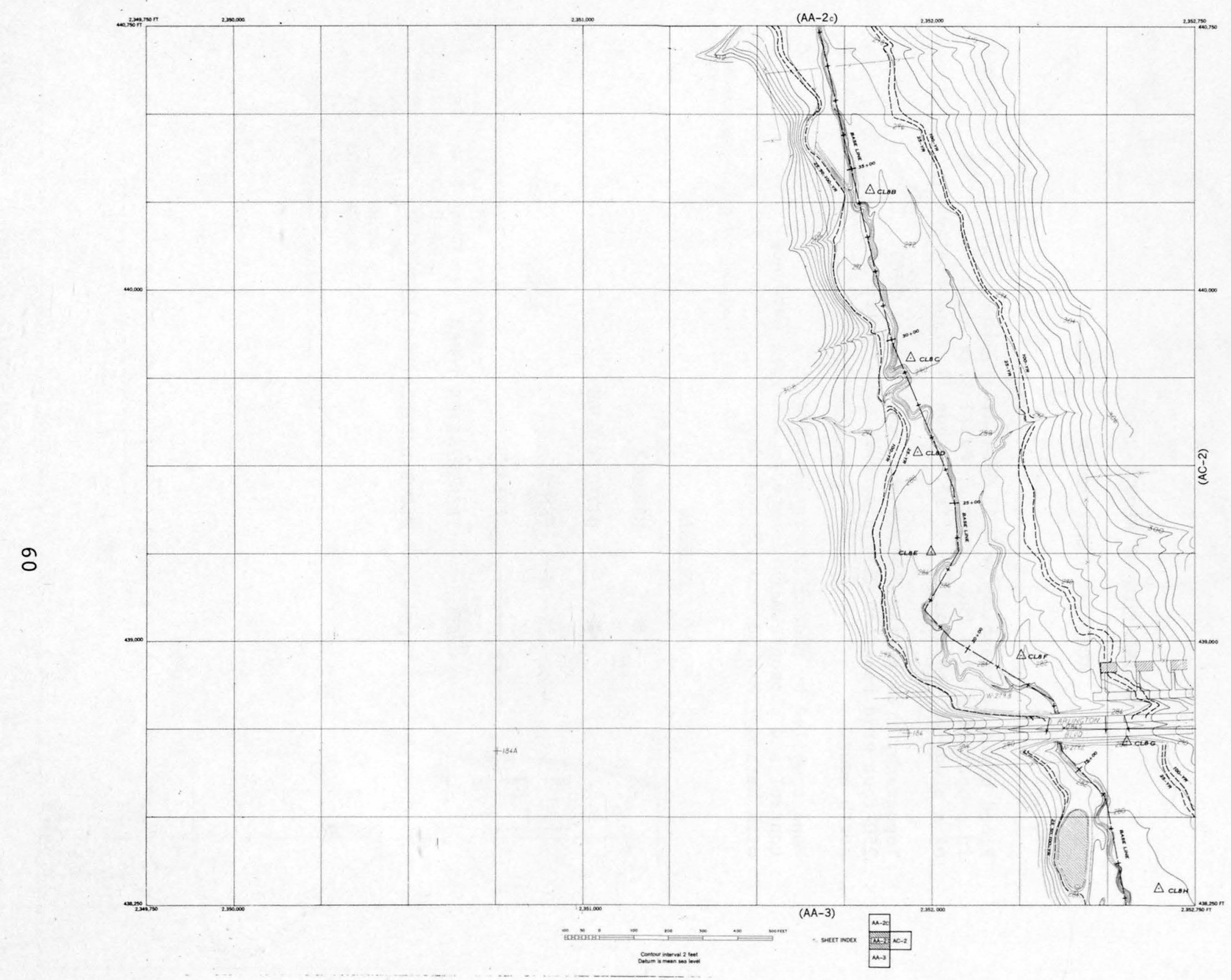

Figure 16.--Topographic map of stream valley showing flood boundary delineation, Accotink Creek; AA-2 


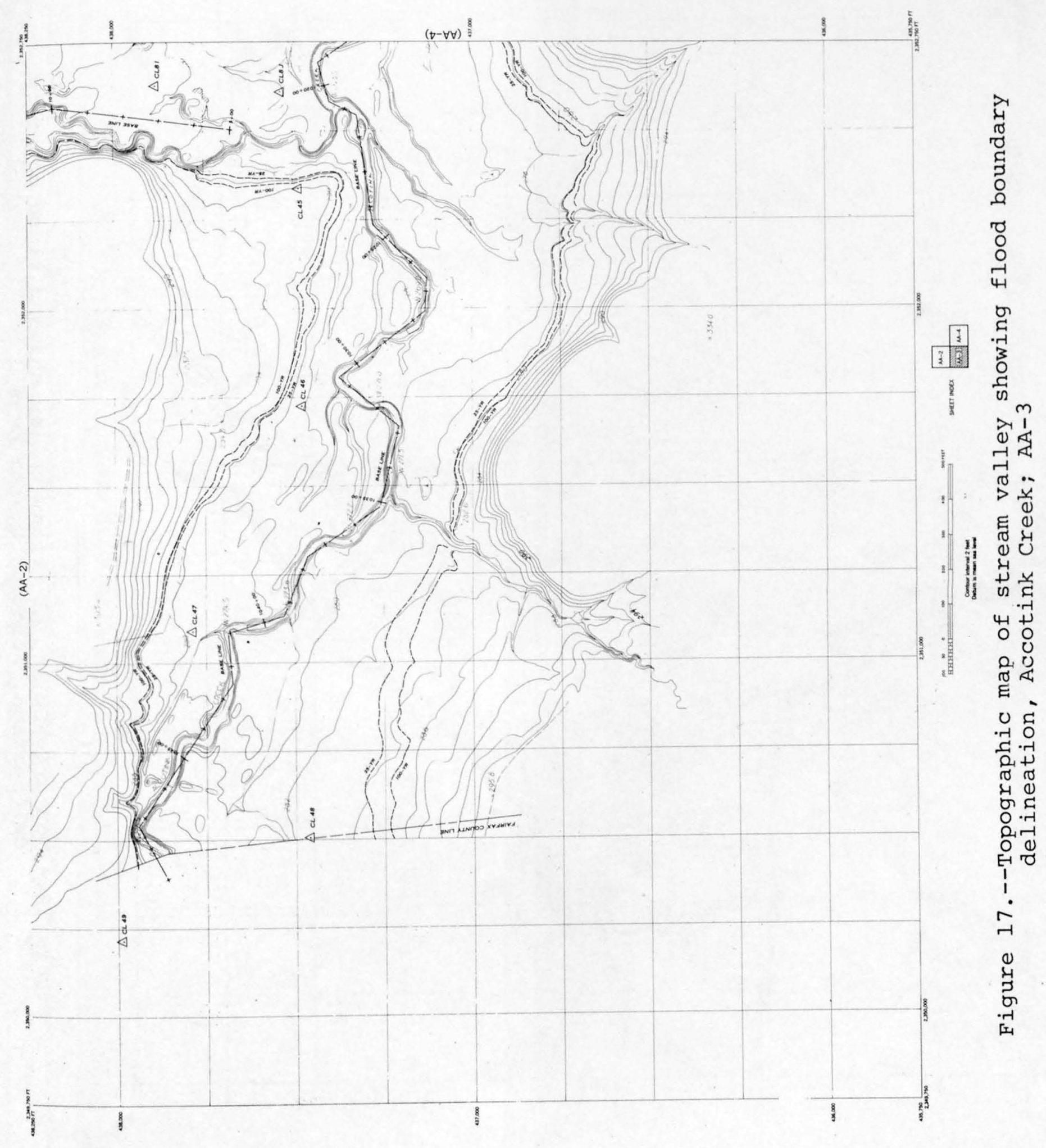




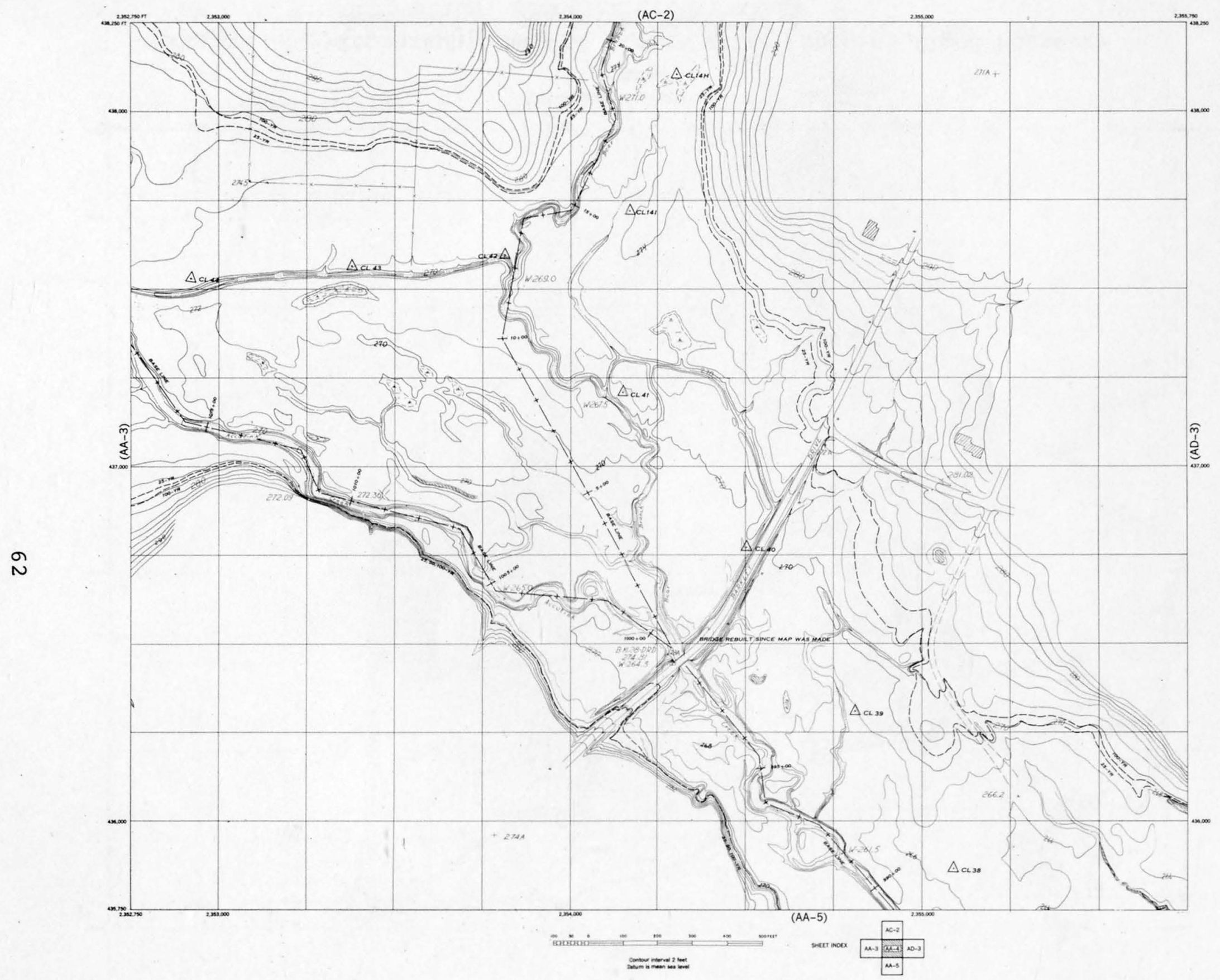

Figure 18.--Topographic map of stream valley showing flood boundary delineation, Accotink Creek; AA-4 
$\stackrel{\sigma}{\omega}$

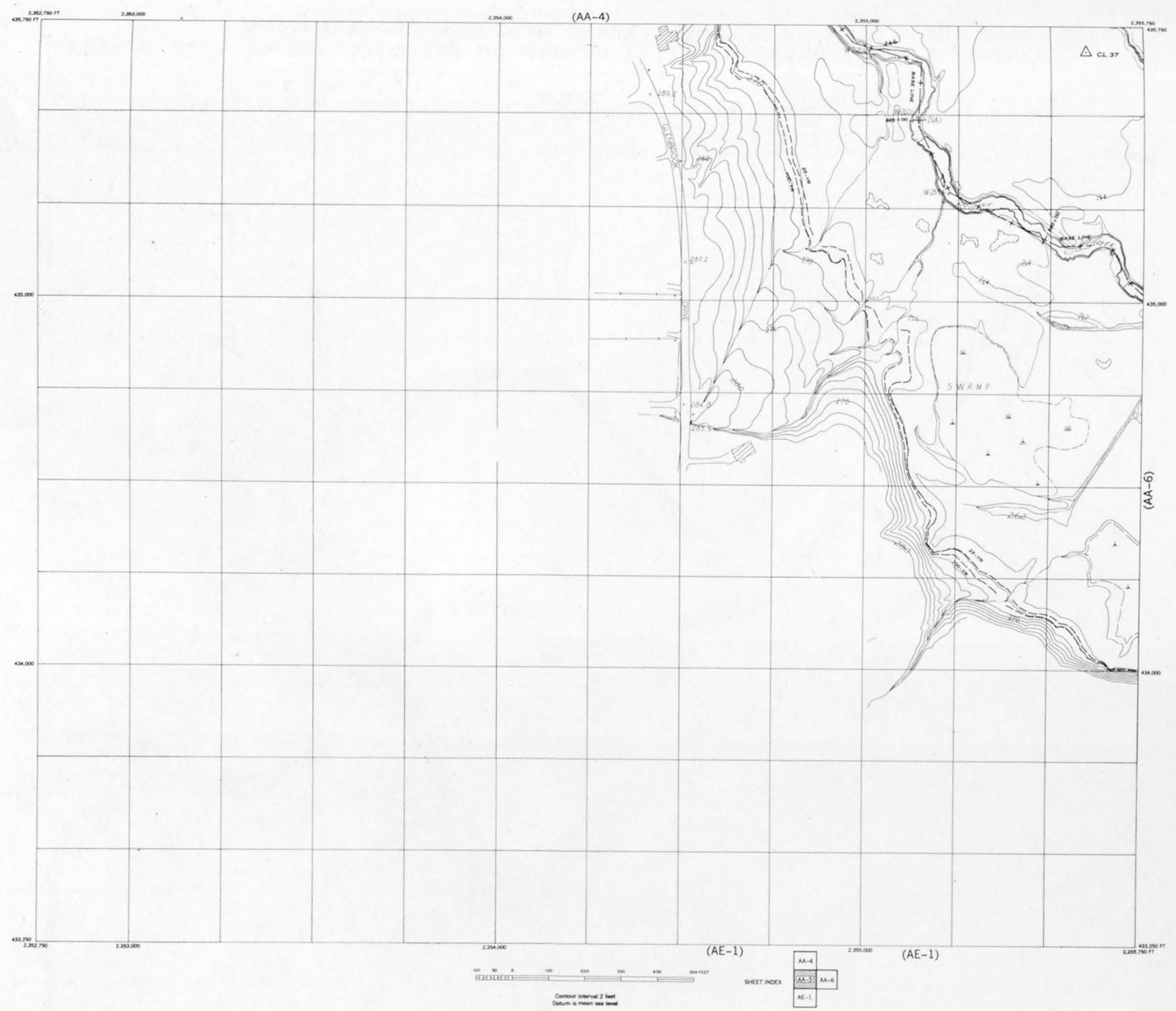

Figure 19.--Topographic map of stream valley showing flood boundary delineation, Accotink Creek; AA-5 


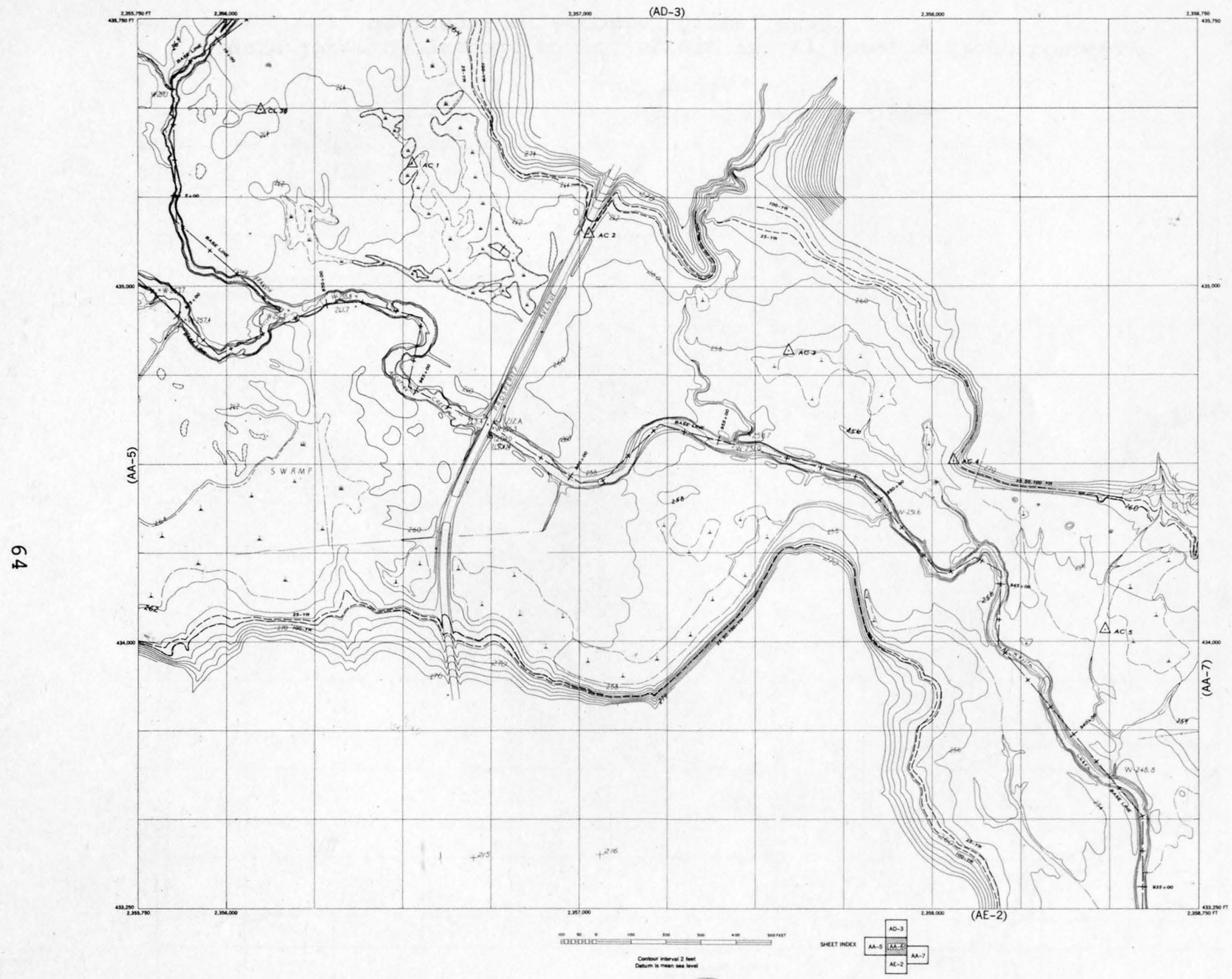

Figure 20.--Topographic map of stream valley showing flood boundary delineation, Accotink Creek; AA-6 


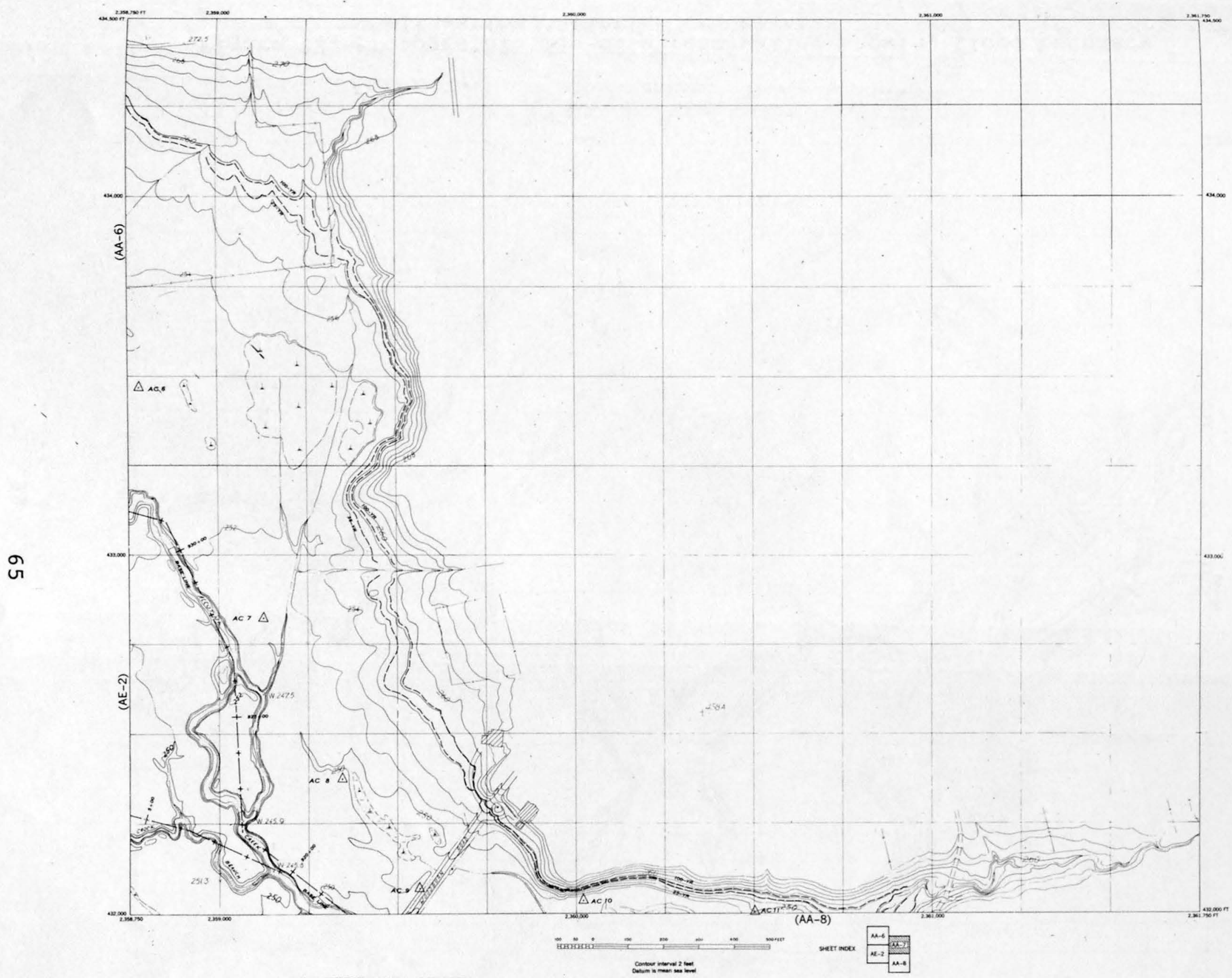

Figure 21.--Topographic map of stream valley showing flood boundary delineation, Accotink Creek; AA -7 


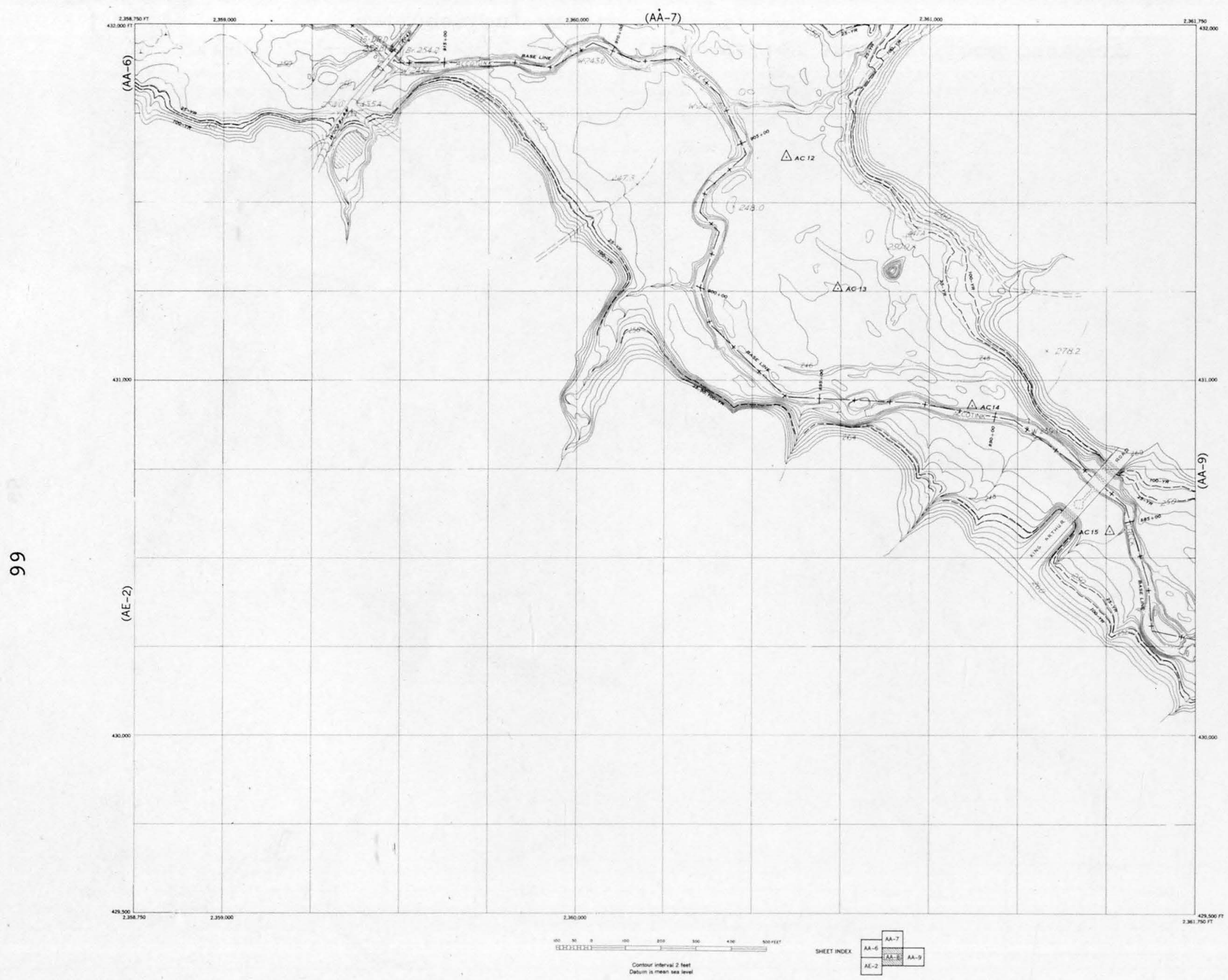

Figure 22.--Topographic map of stream valley showing flood boundary delineation, Accotink Creek; $\mathrm{AA}-8$ 


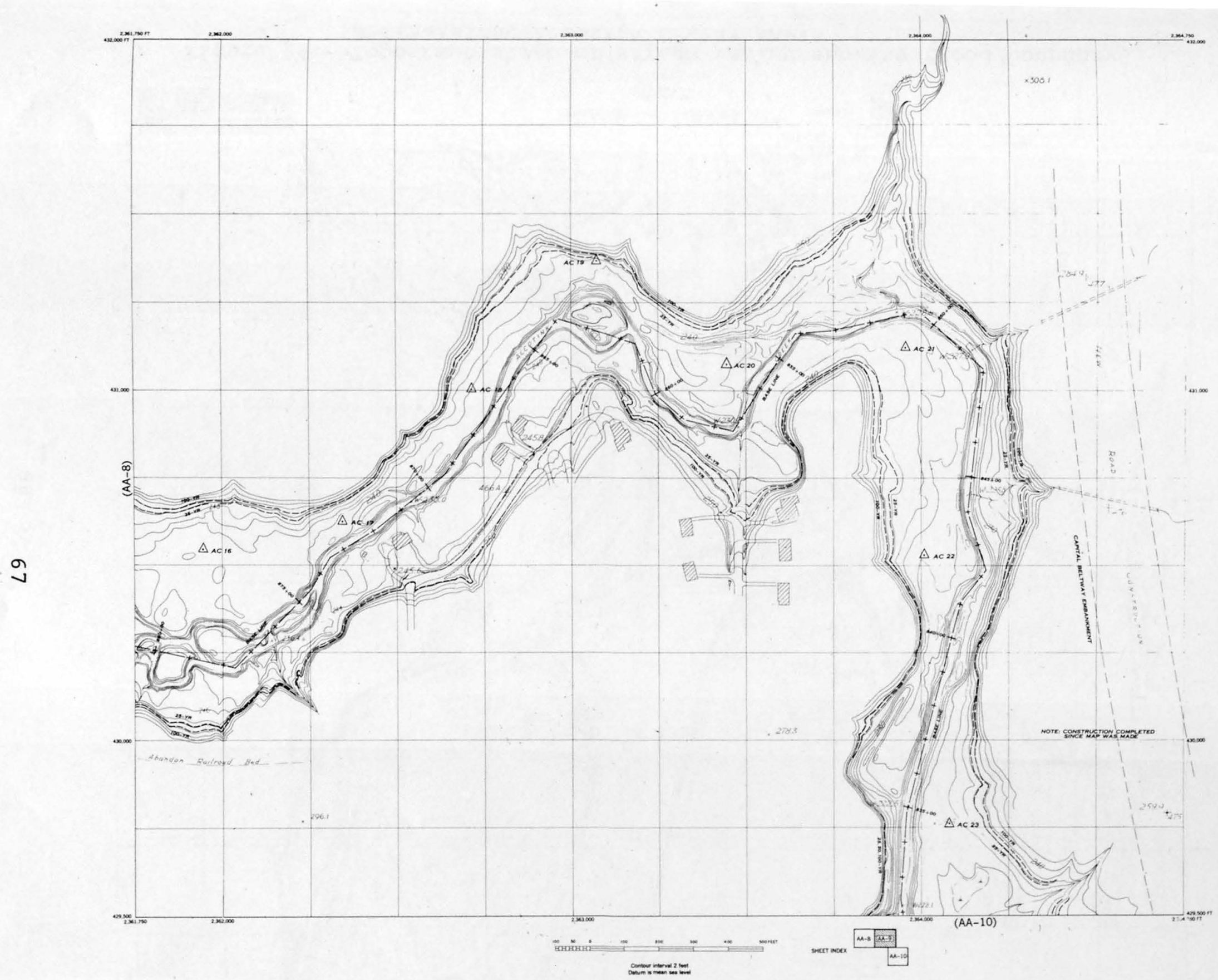

Figure 23.--Topographic map of stream valley showing flood boundary delineation, Accotink Creek; AA-9 


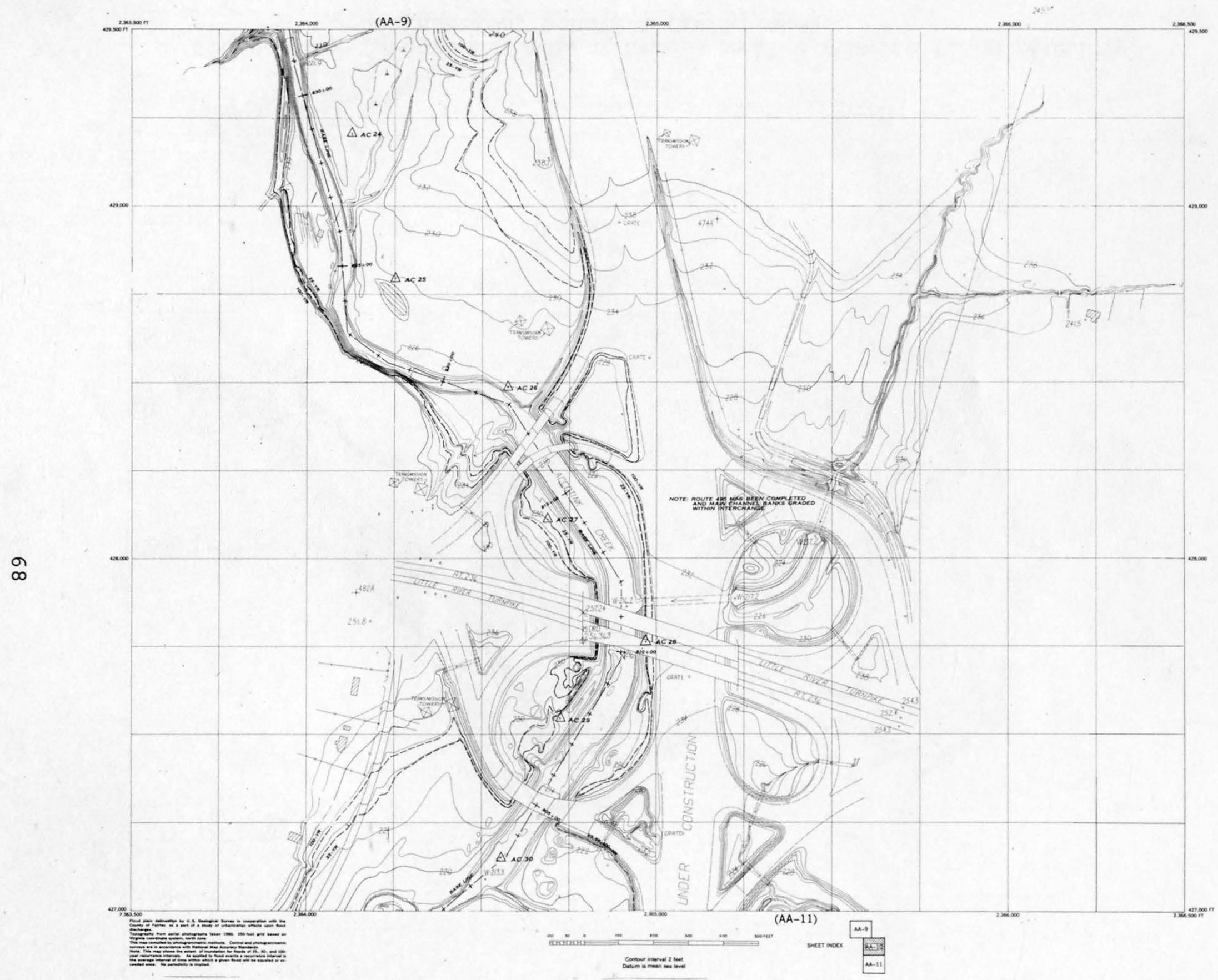

Figure 24.--Topographic map of stream valley showing flood boundary delineation, Accotink Creek; AA-10 


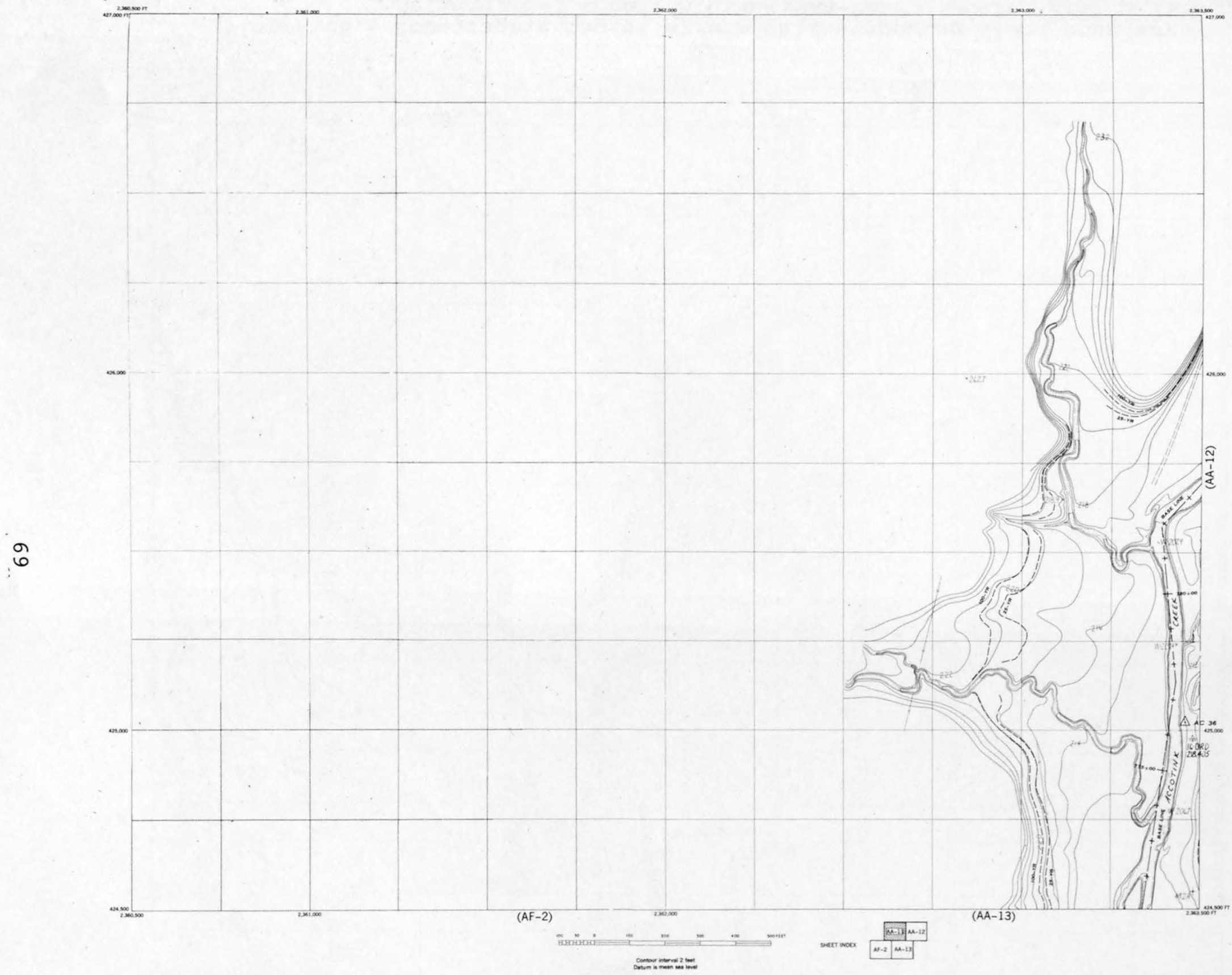

Figure 25.--Topographic map of stream valley showing flood boundary delineation, Accotink Creek; AA-1l 


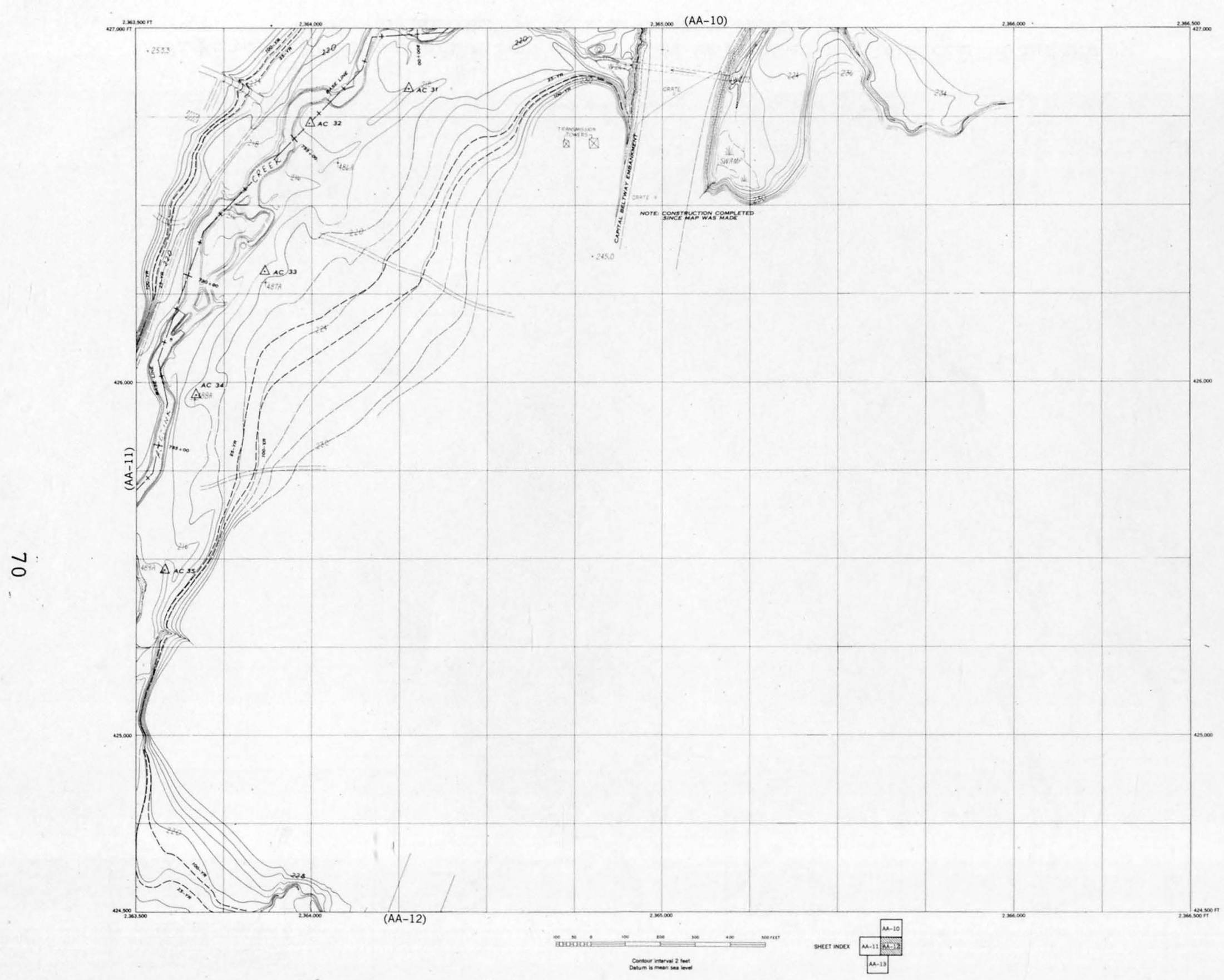

Figure 26.--Topographic map of stream valley showing flood boundary delineation, Accotink Creek; $A A-12$ 


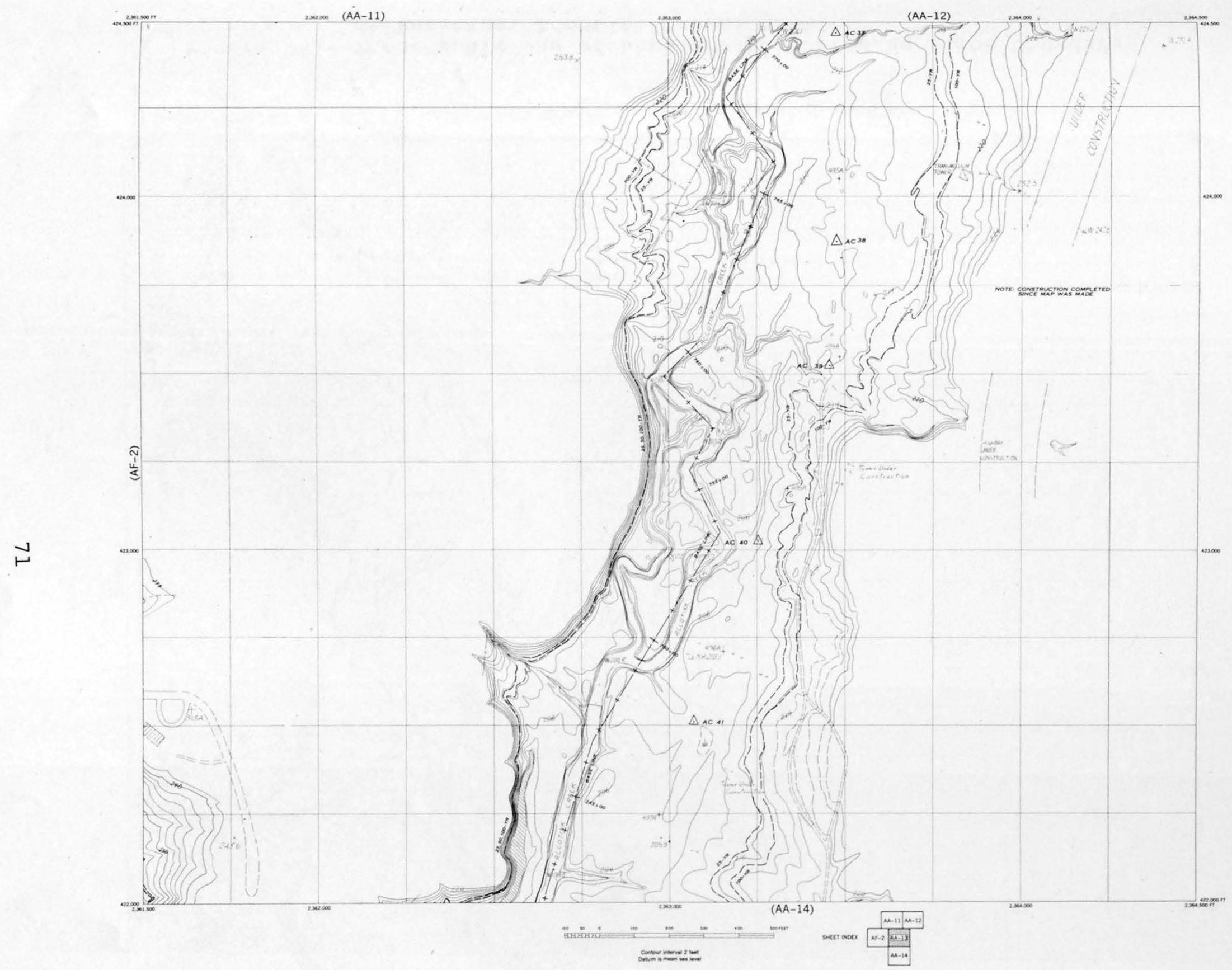

Figure 27.--Topographic map of stream valley showing flood boundary delineation, Accotink Creek; AA-13 


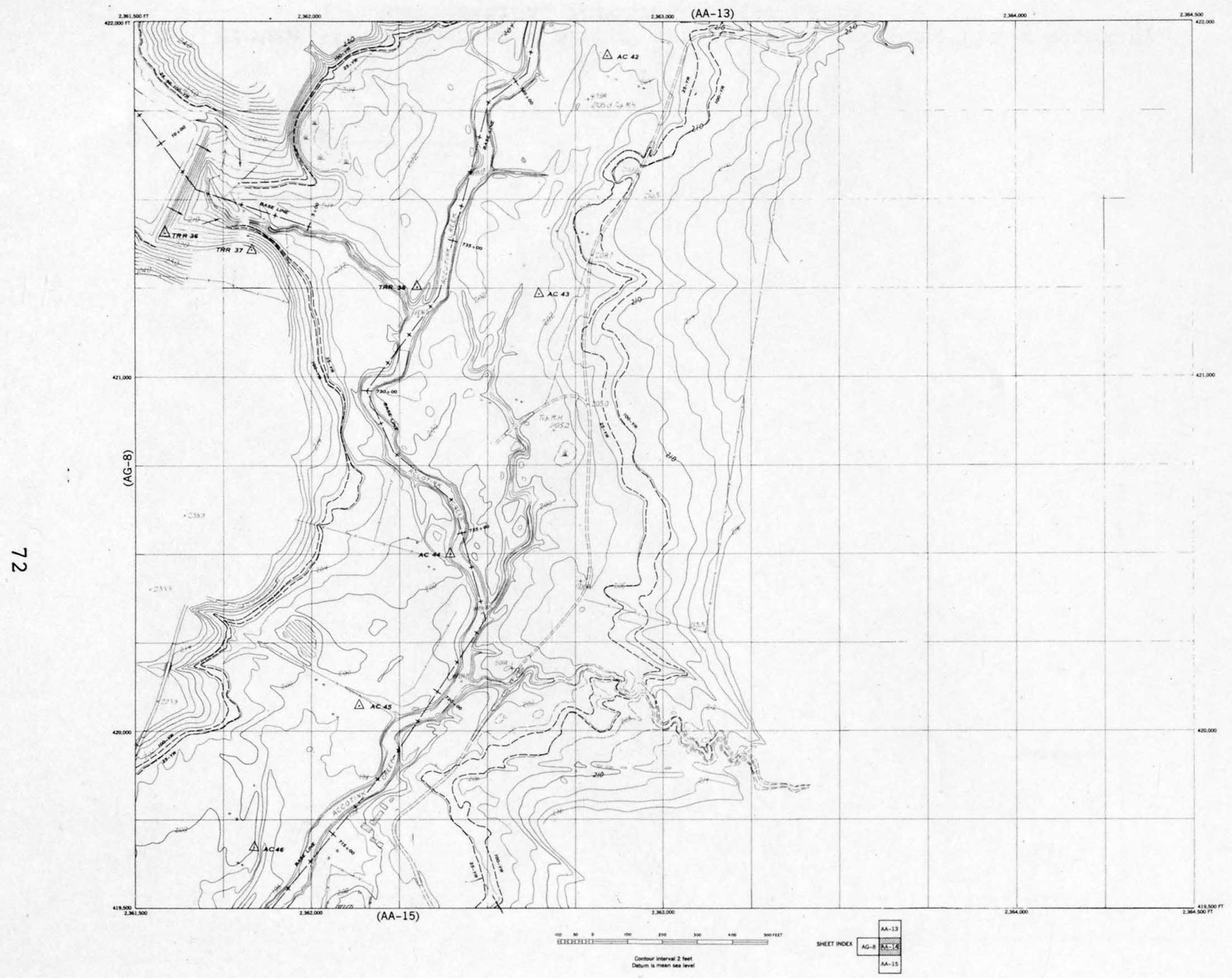

Figure 28.--Topographic map of stream valley showing flood boundary delineation, Accotink Creek; AA-14 


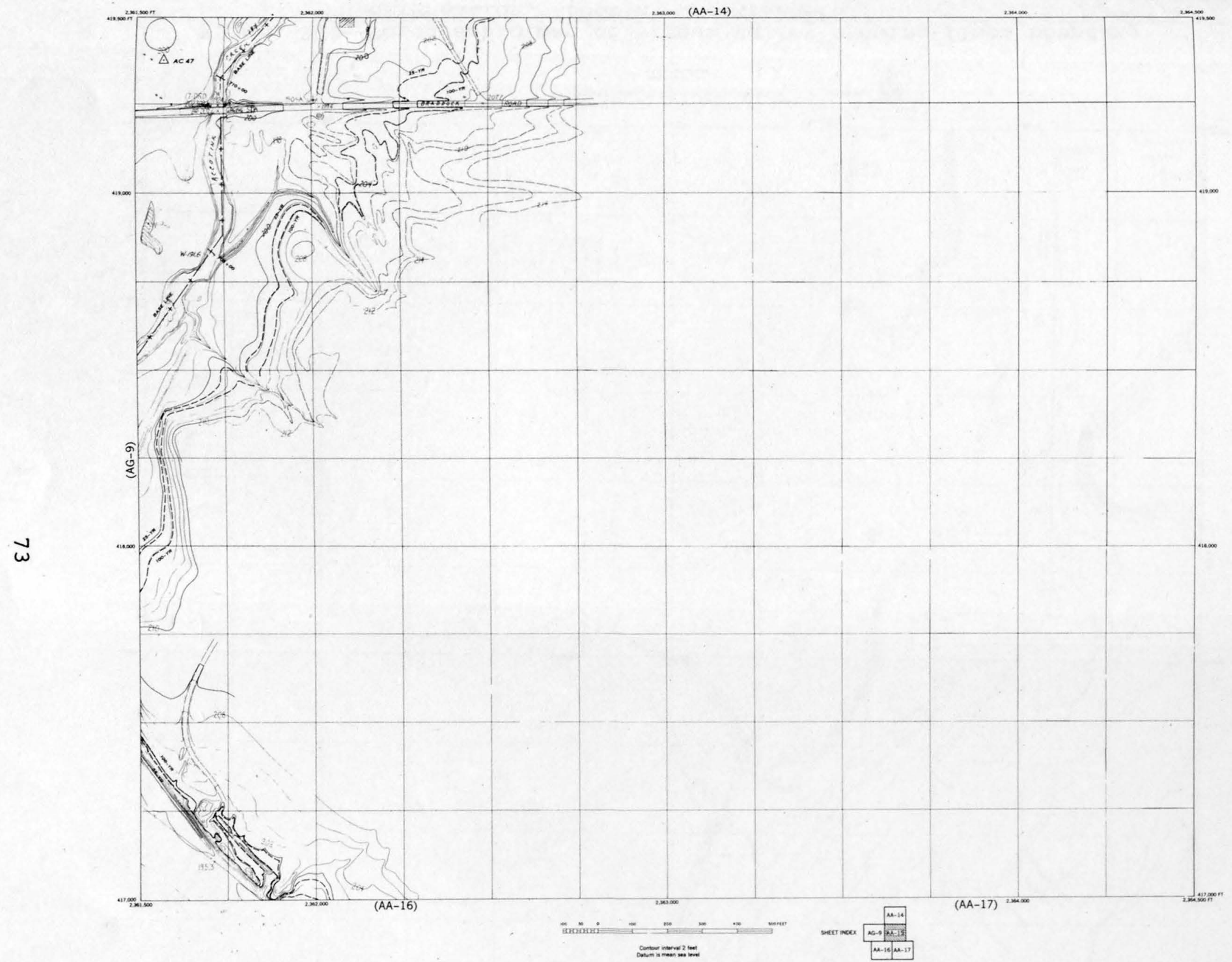

Figure 29.--Topographic map of stream valley showing flood boundary delineation, Accotink Creek; AA-15 


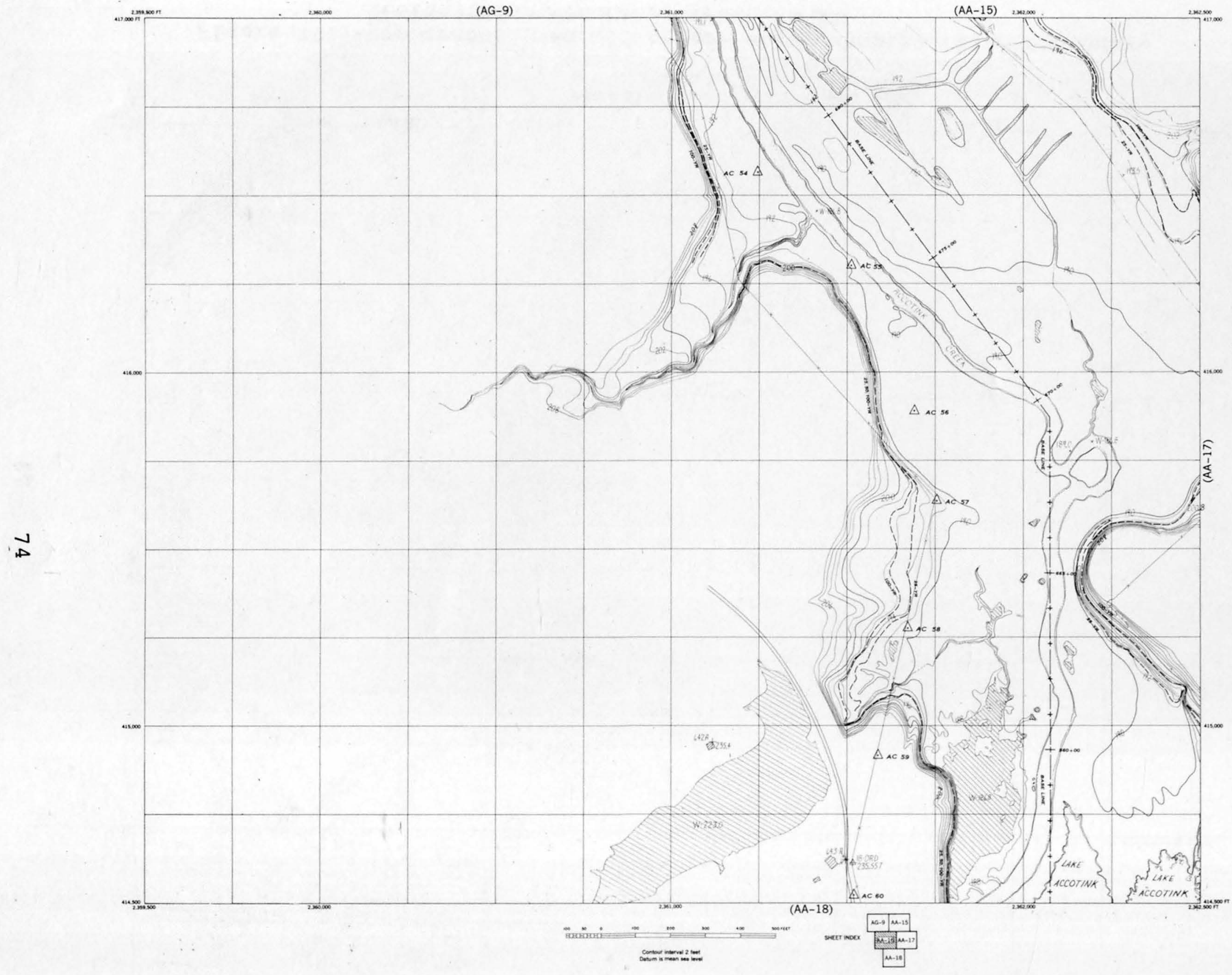

Figure 30.--Topographic map of stream valley showing flood boundary delineation, Accotink Creek; $A A-16$ 


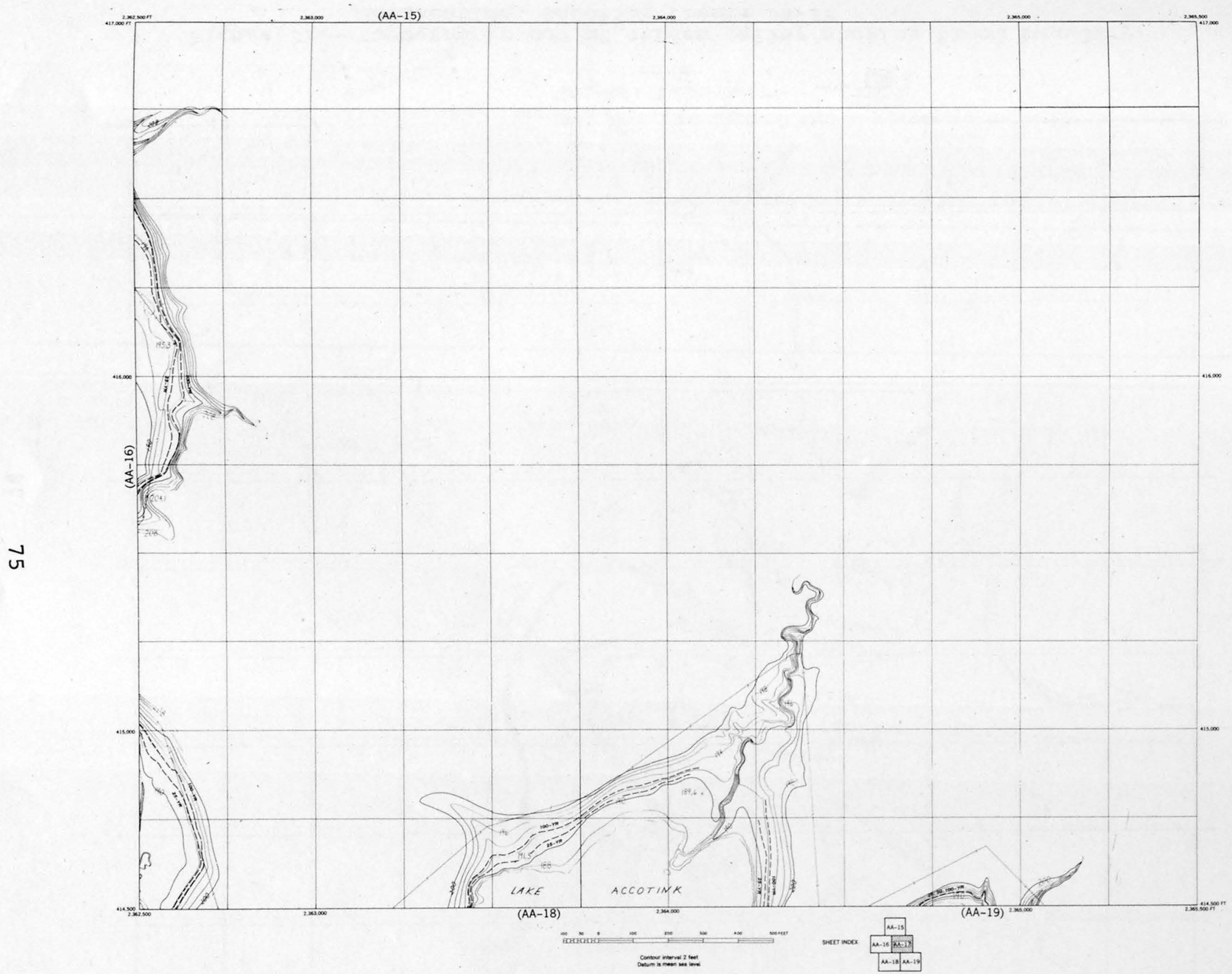

Figure 31.--Topographic map of stream valley showing flood boundary delineation, Accotink Creek; $\mathrm{AA}-17$ 


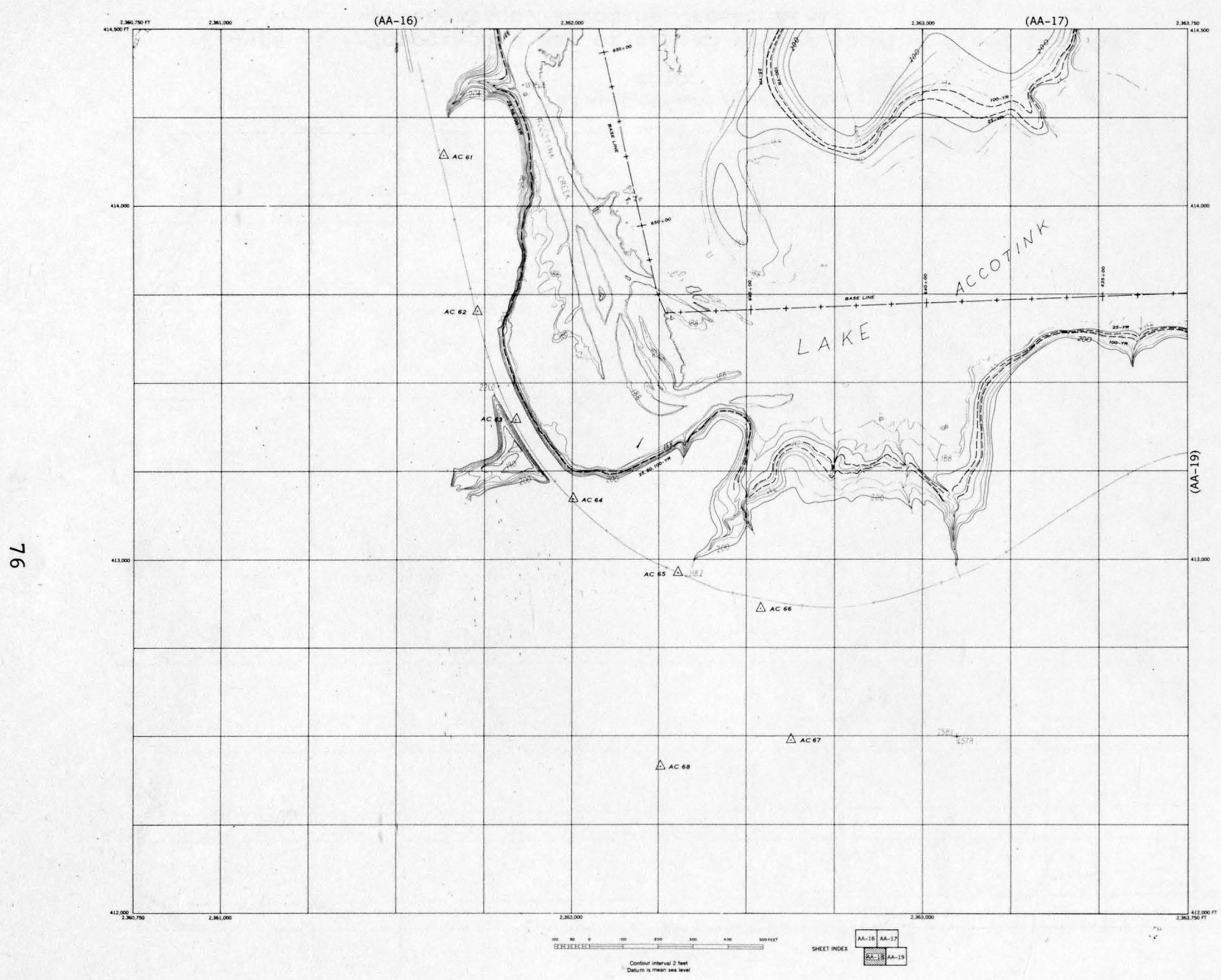

Figure 32.--Topographic map of stream valley showing flood boundary delineation, Accotink Creek; AA-18 


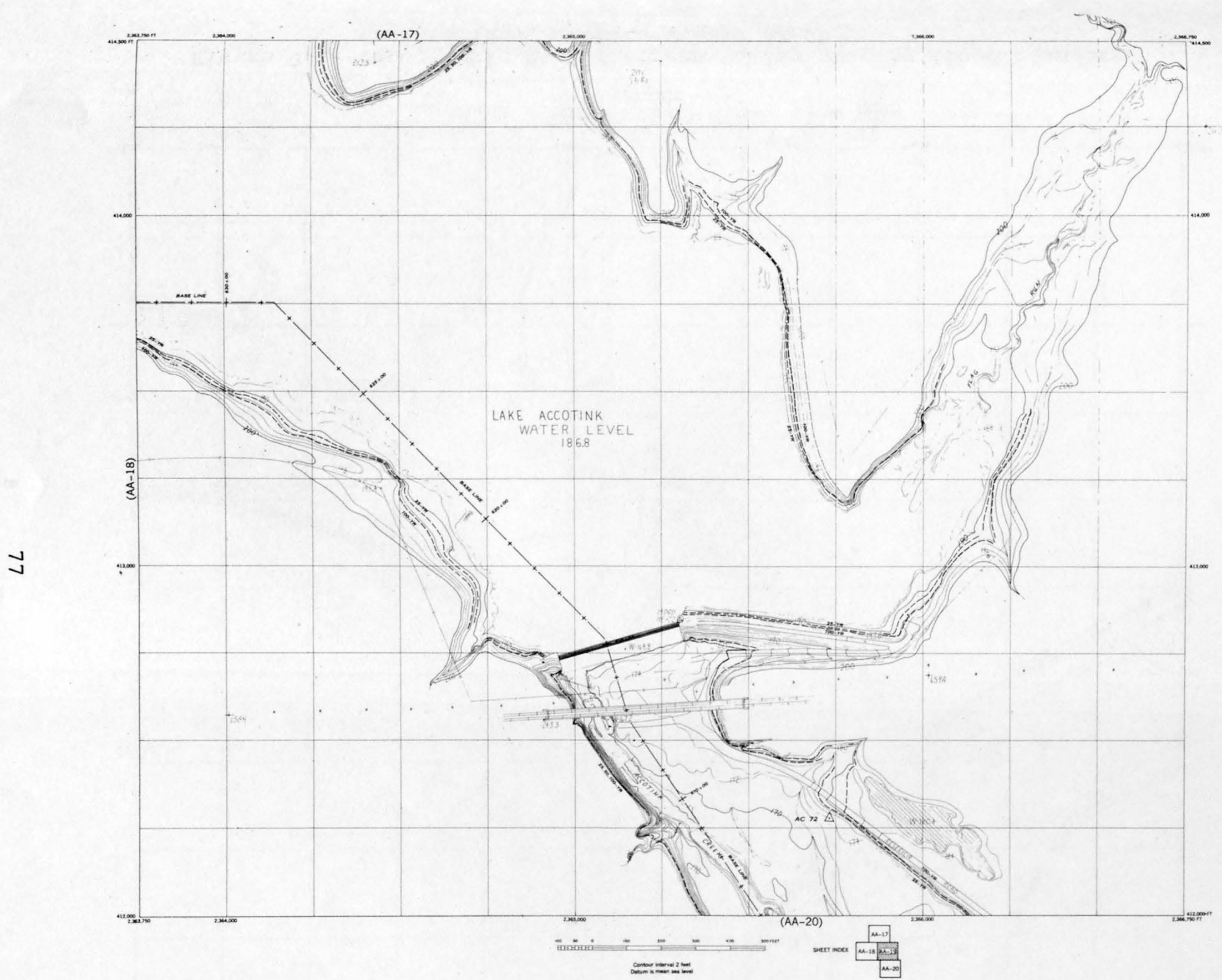

Figure 33.--Topographic map of stream valley showing flood boundary delineation, Accotink Creek; AA-19 


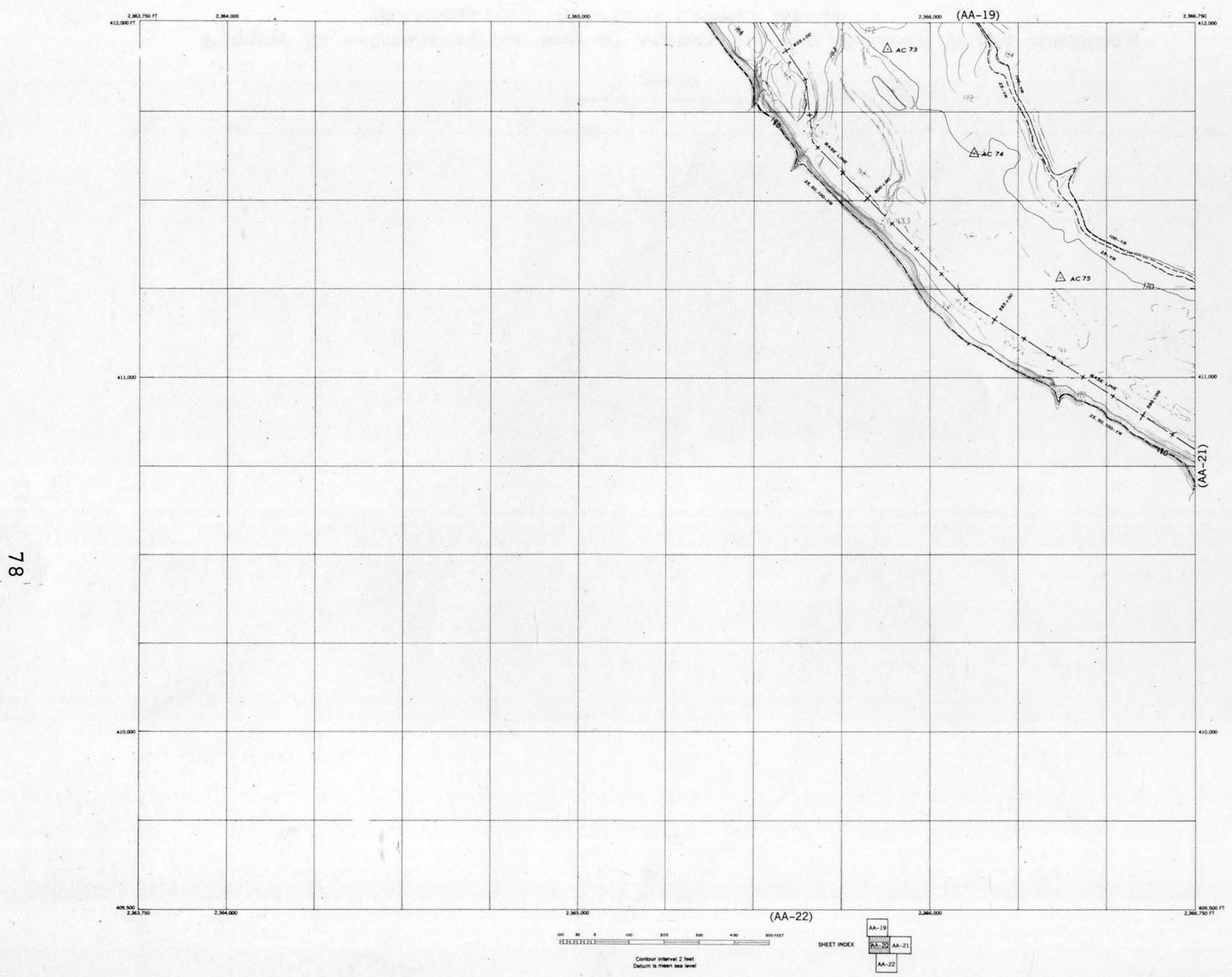

Figure 34.--Topographic map of stream valley showing flood boundary delineation, Accotink Creek; AA-20 


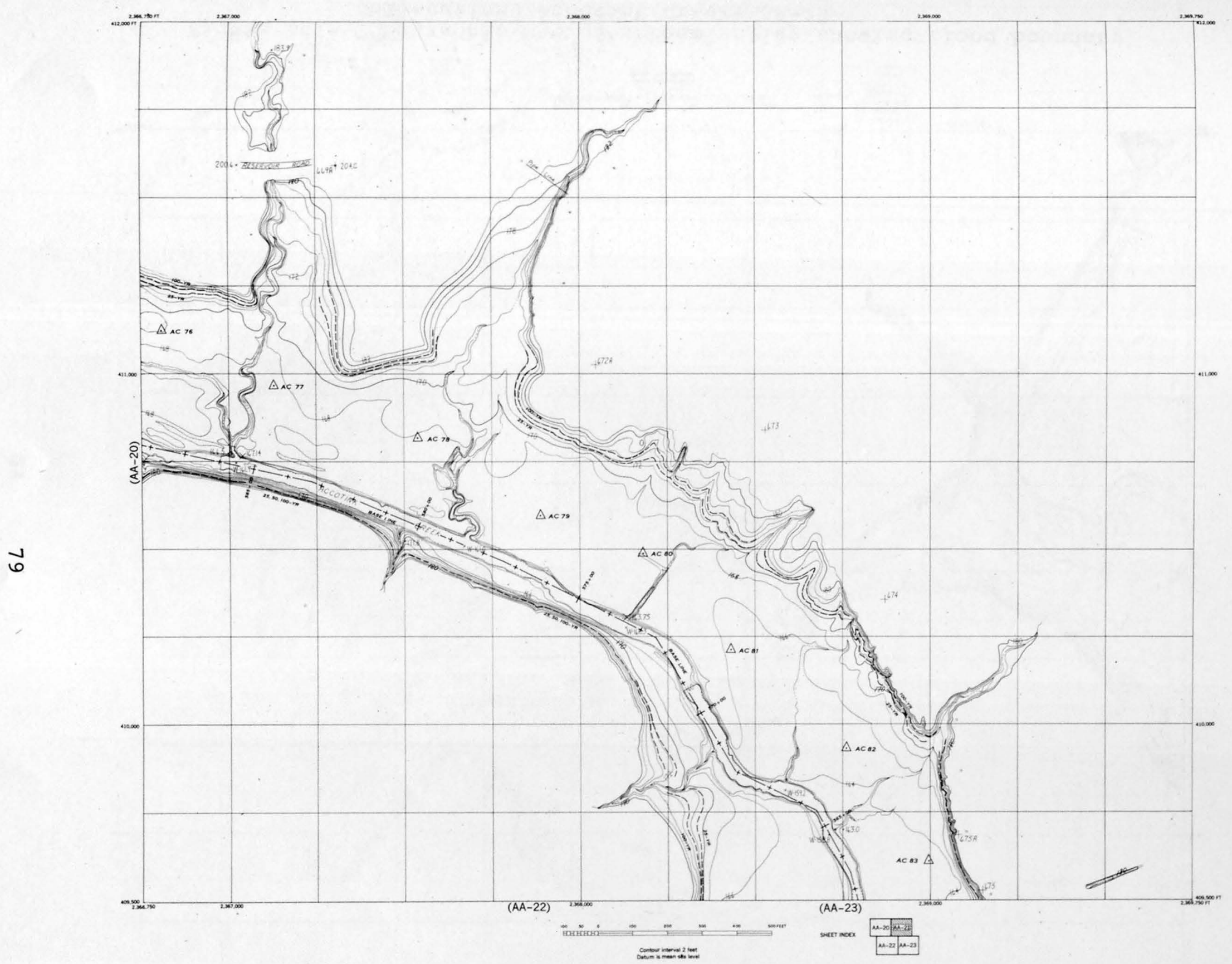

Figure 35.--Topographic map of stream valley showing flood boundary delineation, Accotink Creek; $\mathrm{AA}-21$ 


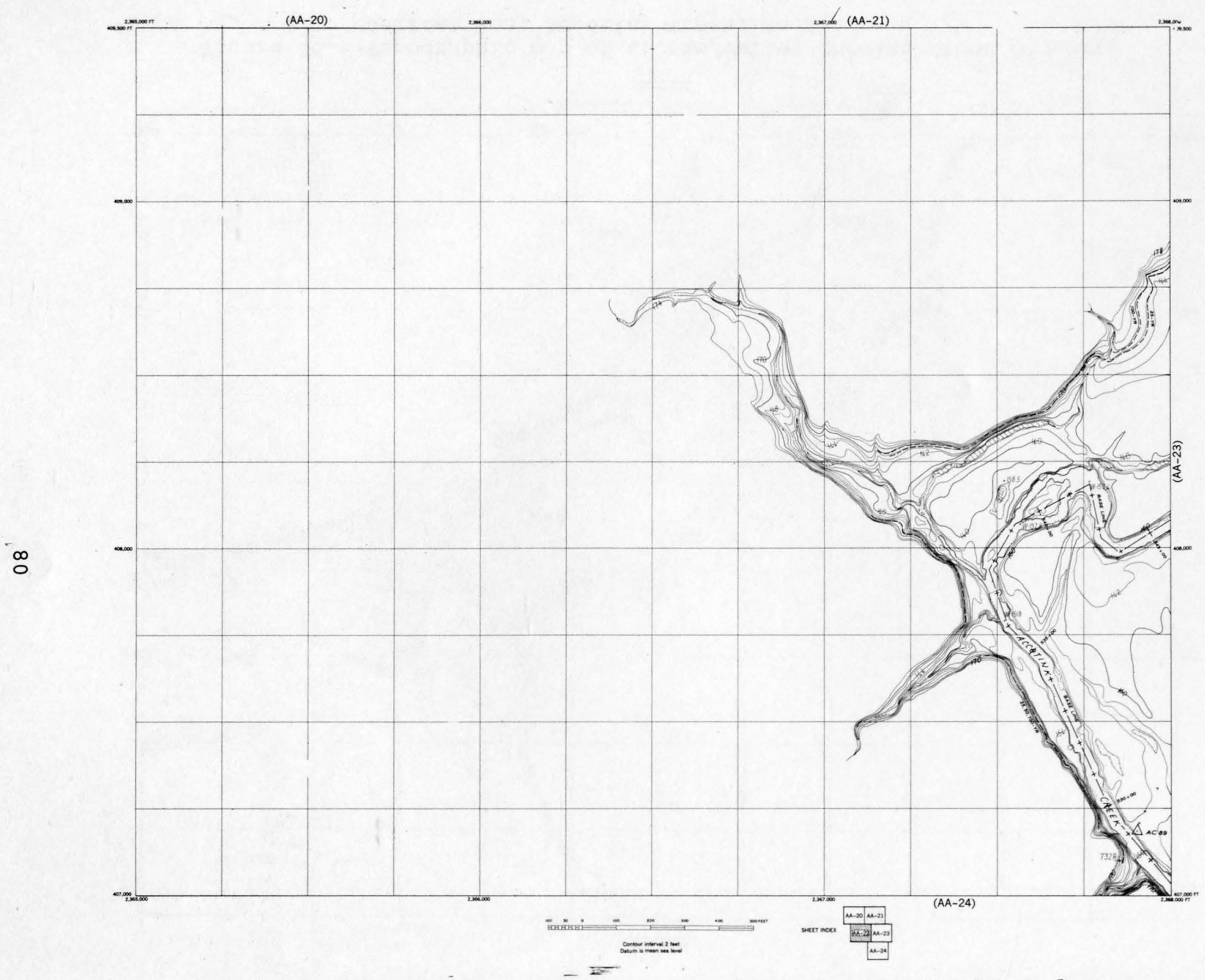

Figure 36.--Topographic map of stream valley showing flood boundary delineation, Accotink Creek; AA-22 


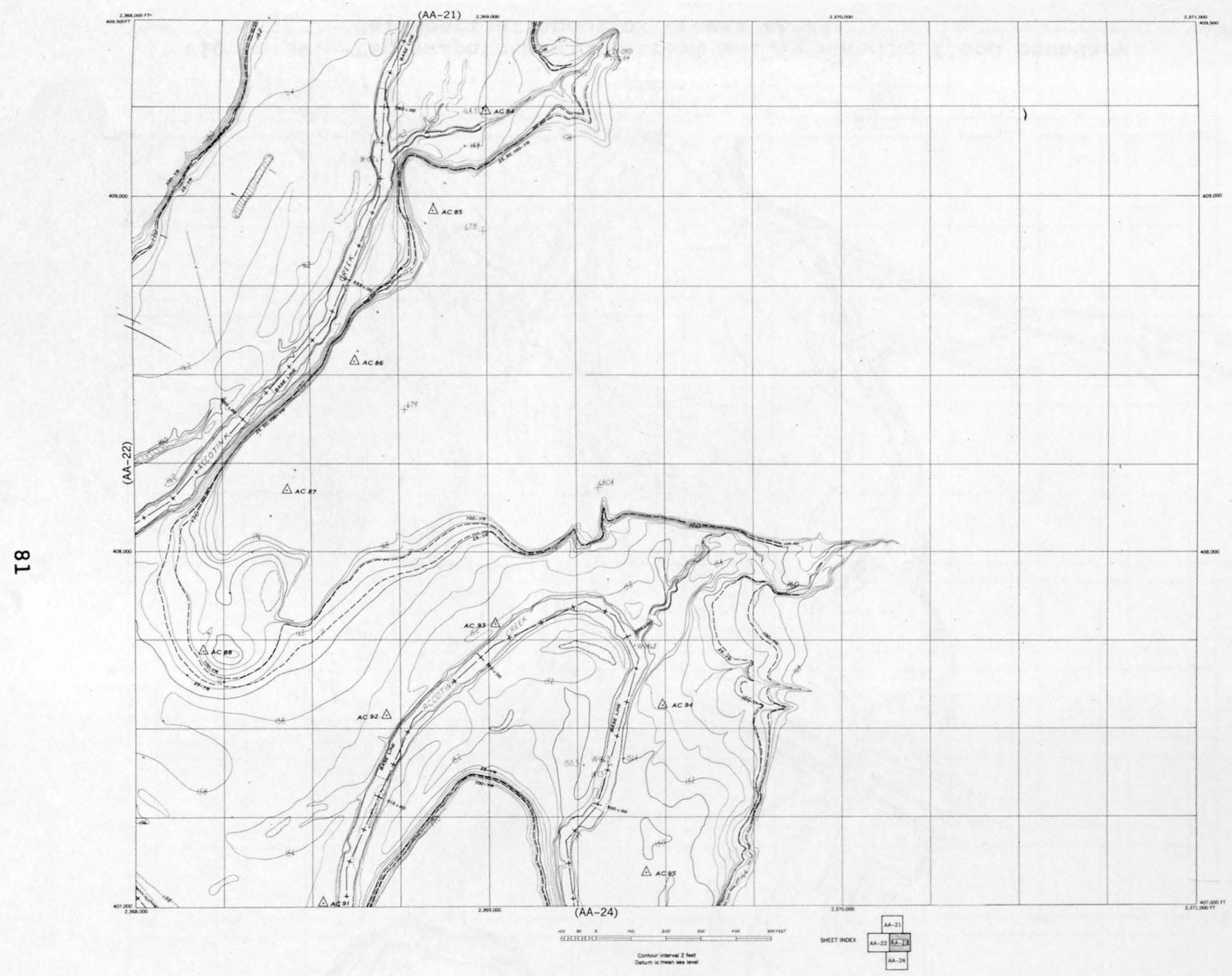

Figure 37.--Topographic map of stream valley showing flood boundary delineation, Accotink Creek; $\mathrm{AA}-23$ 


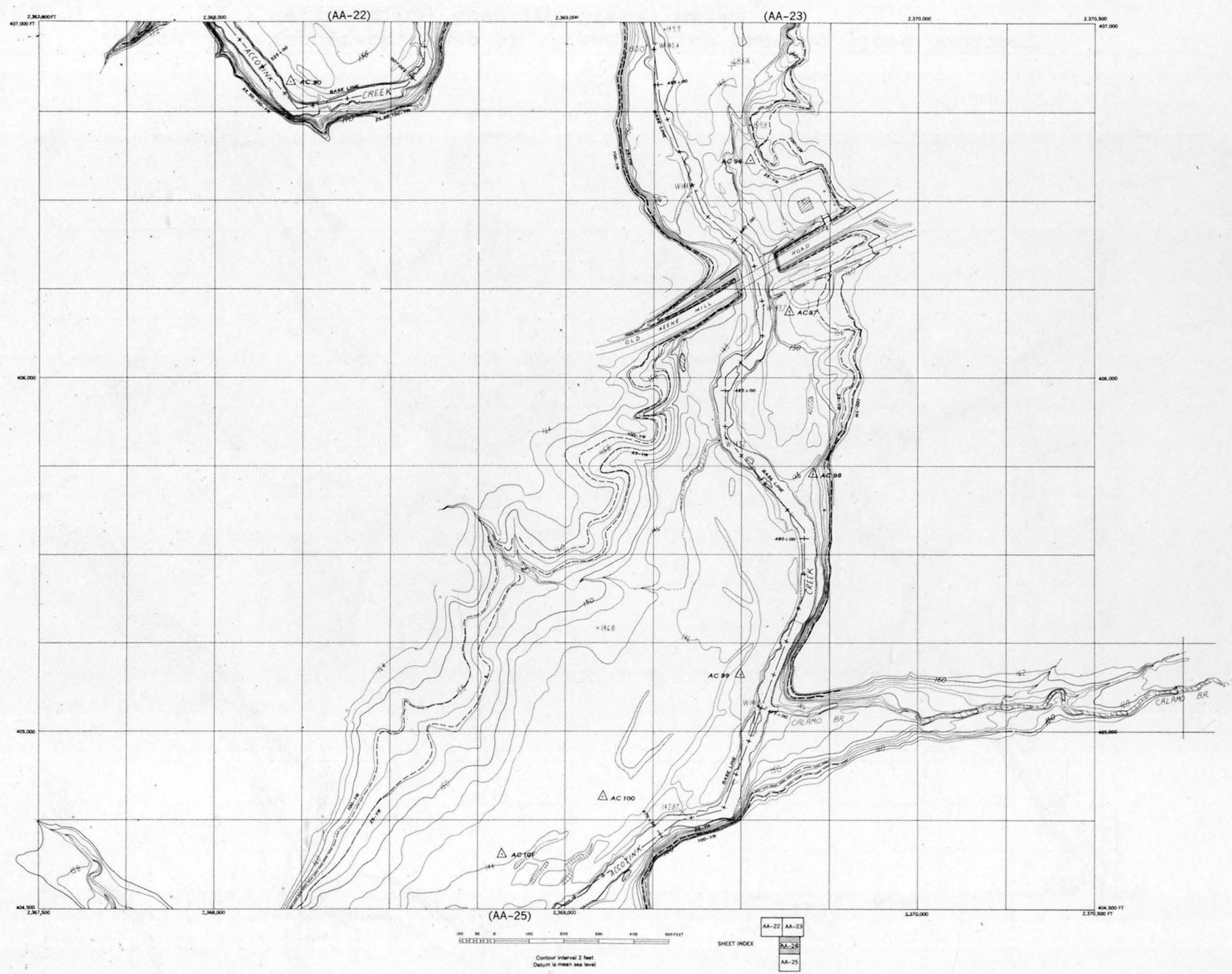

Figure 38.--Topographic map of stream valley showing flood boundary delineation, Accotink Creek; AA-24 


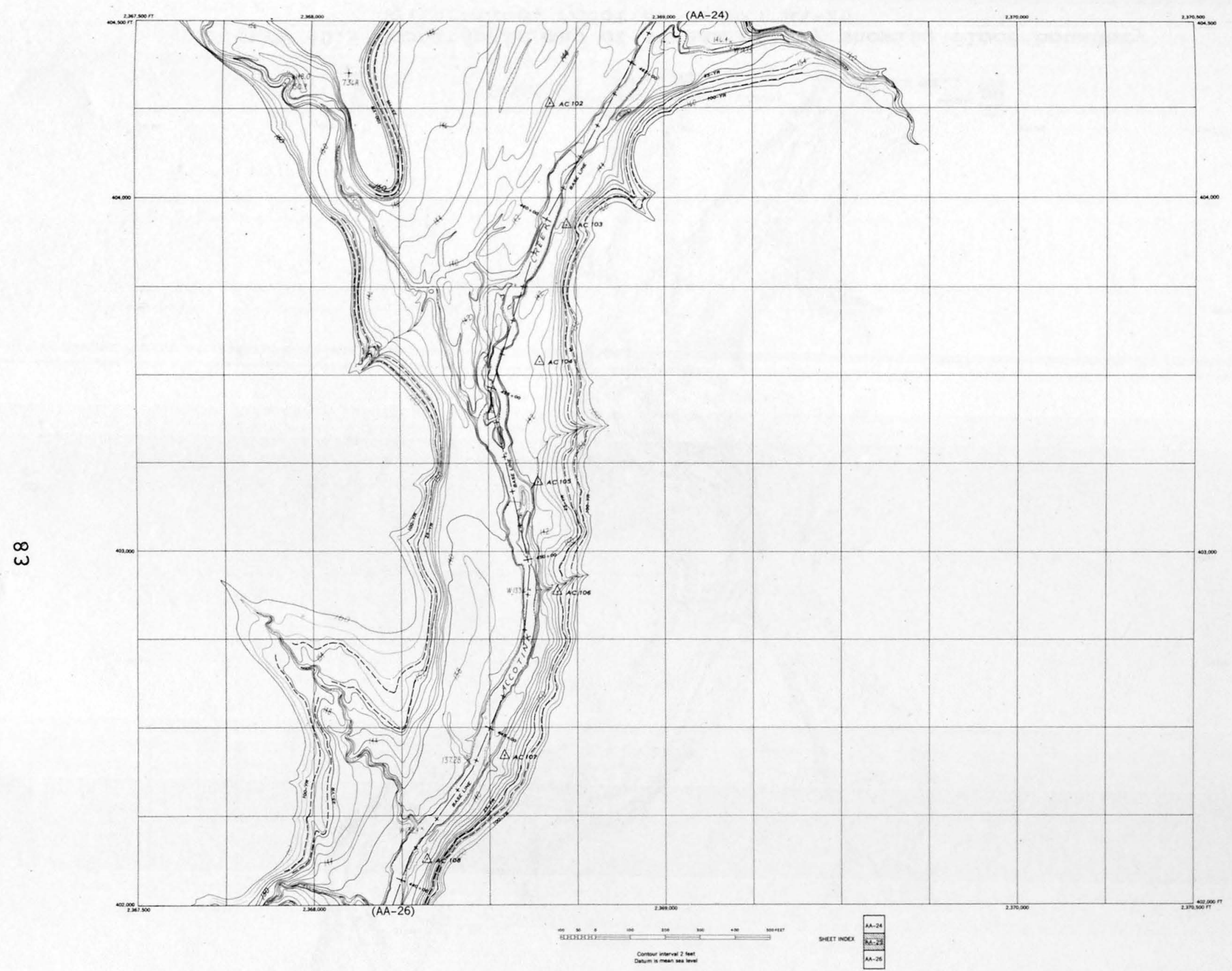

Figure 39.--Topographic map of stream valley showing flood boundary delineation, Accotink Creek; AA-25 


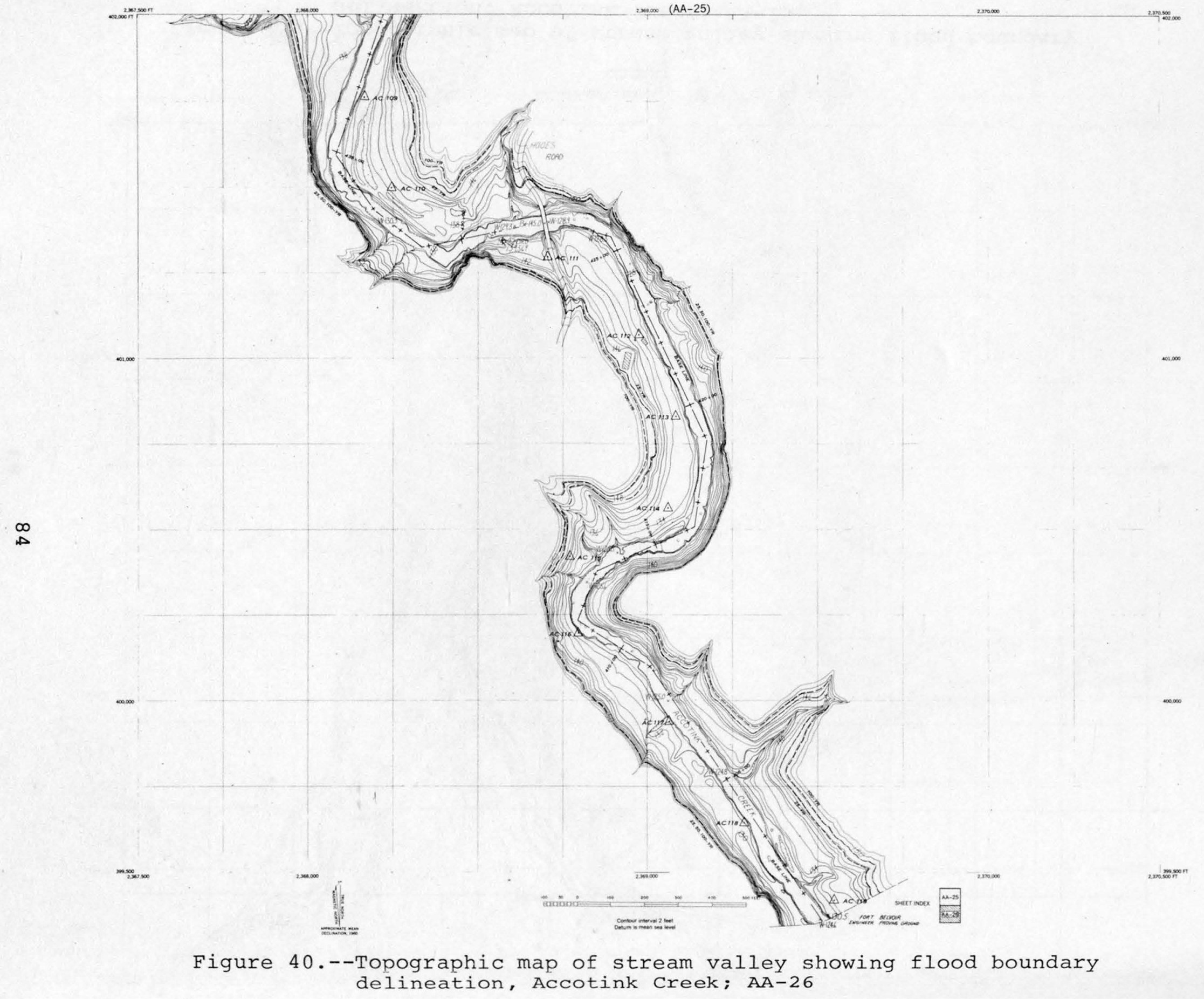




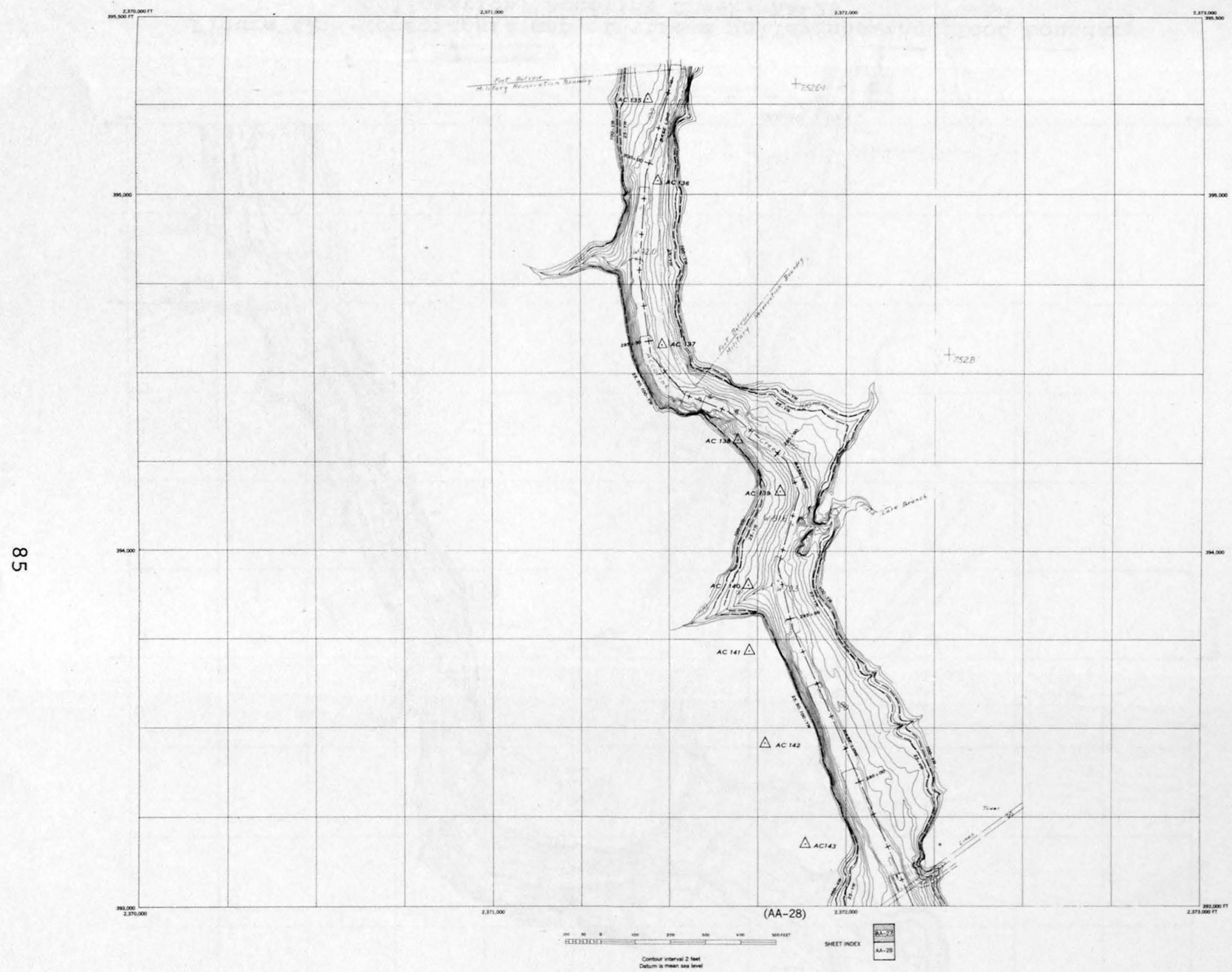

Figure 41.--Topographic map of stream valley showing flood boundary delineation, Accotink Creek; AA-27 


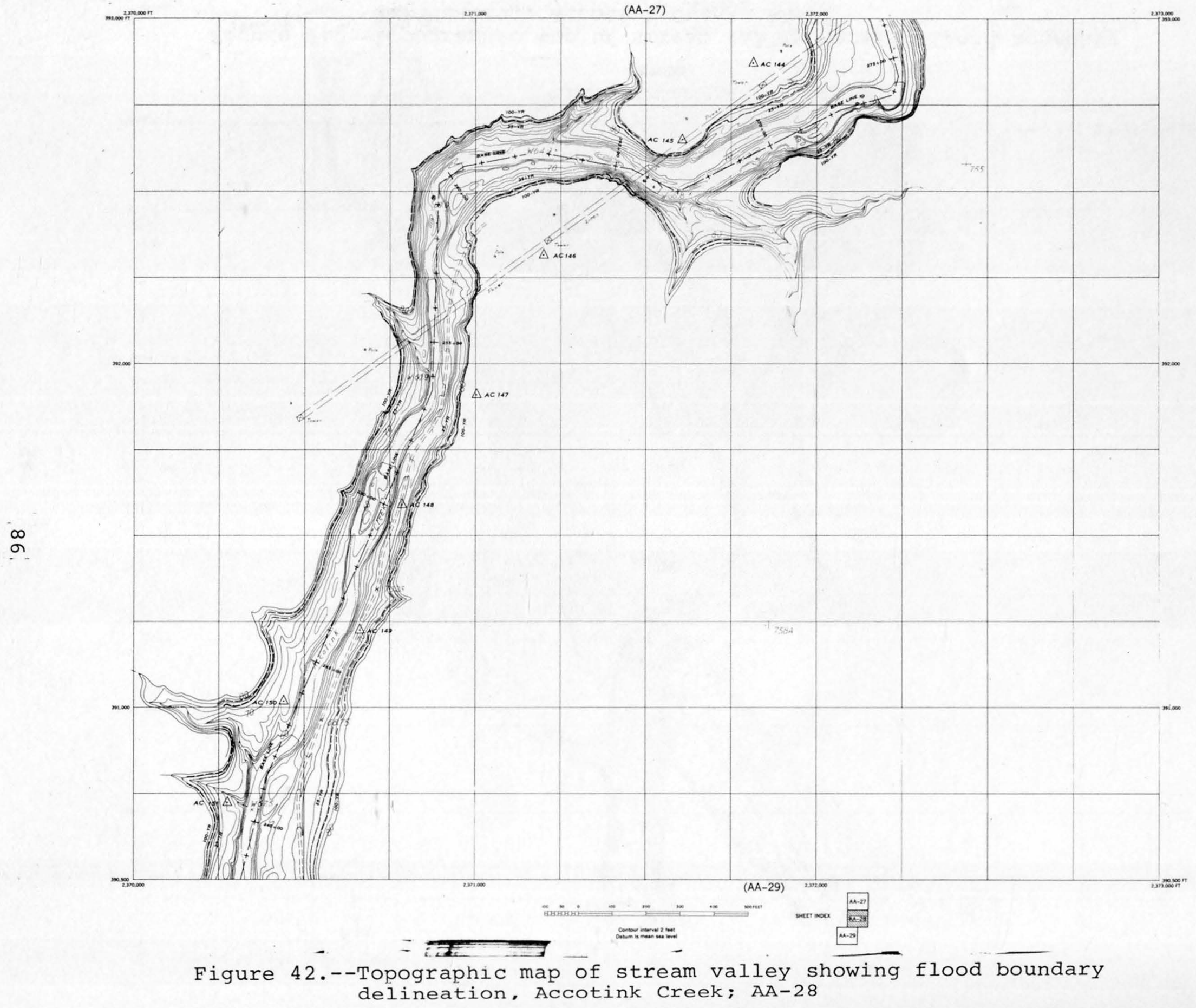




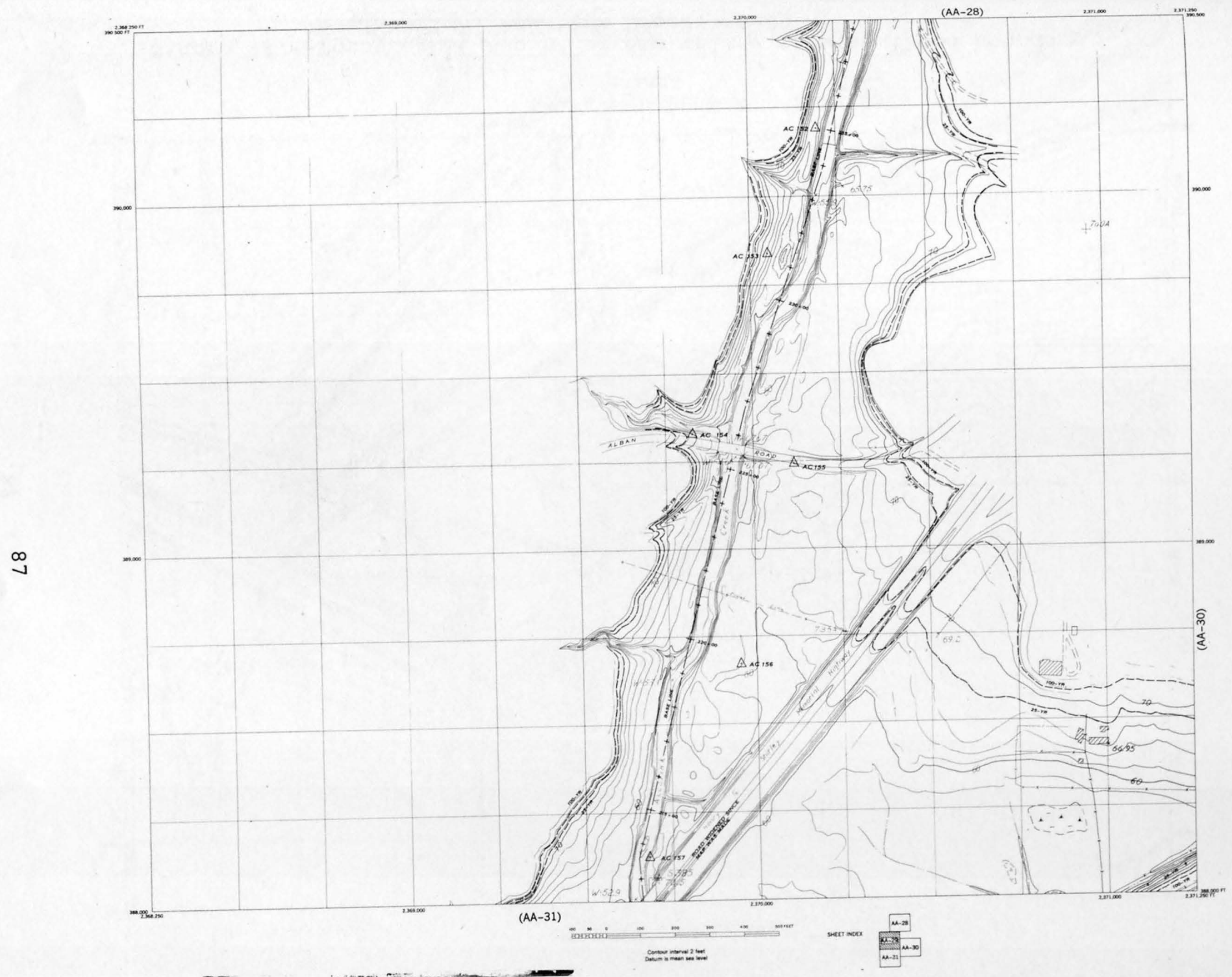

Figure 43.--Topographic map of stream valley showing flood boundary delineation, Accotink Creek; AA-29 


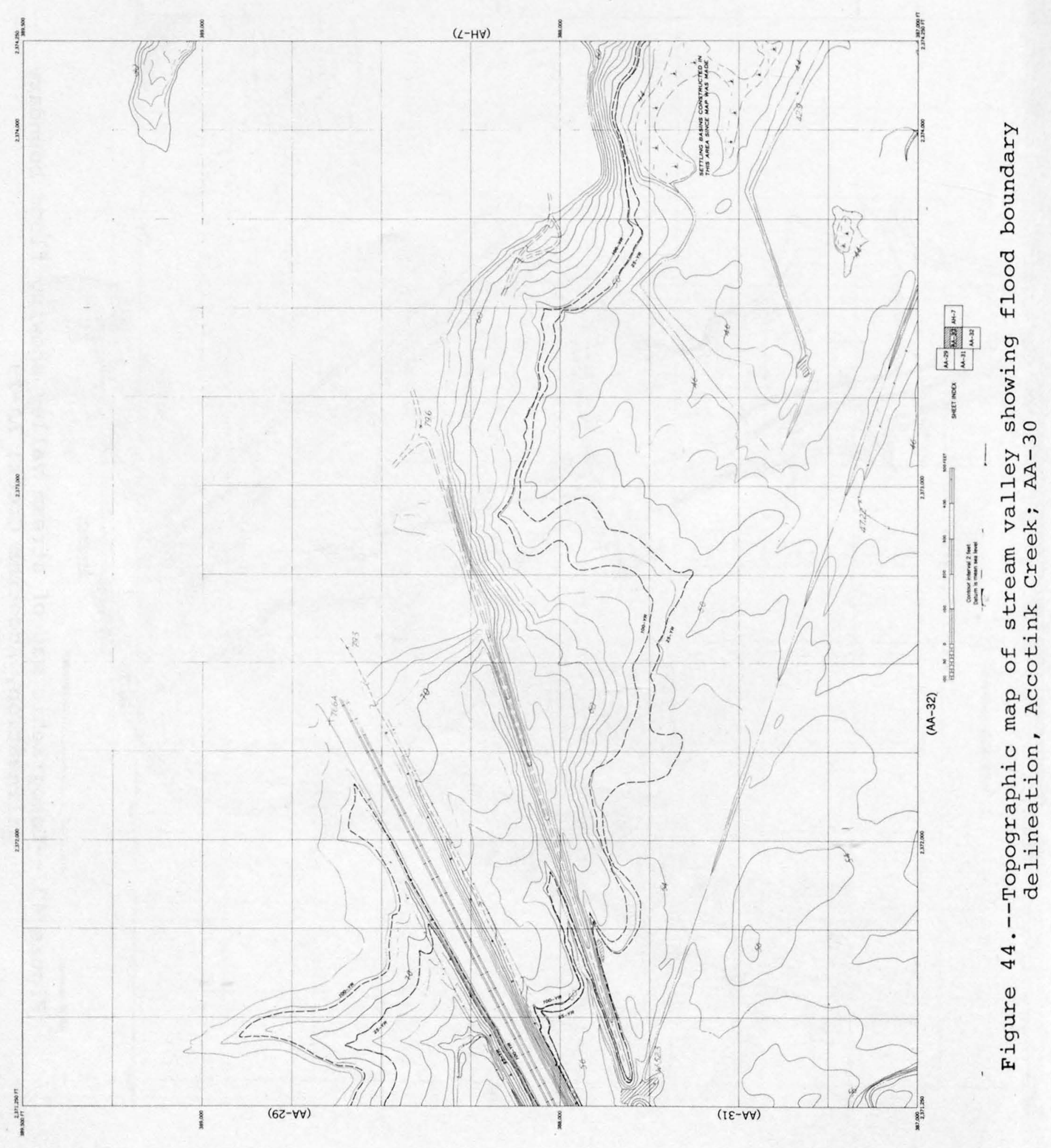




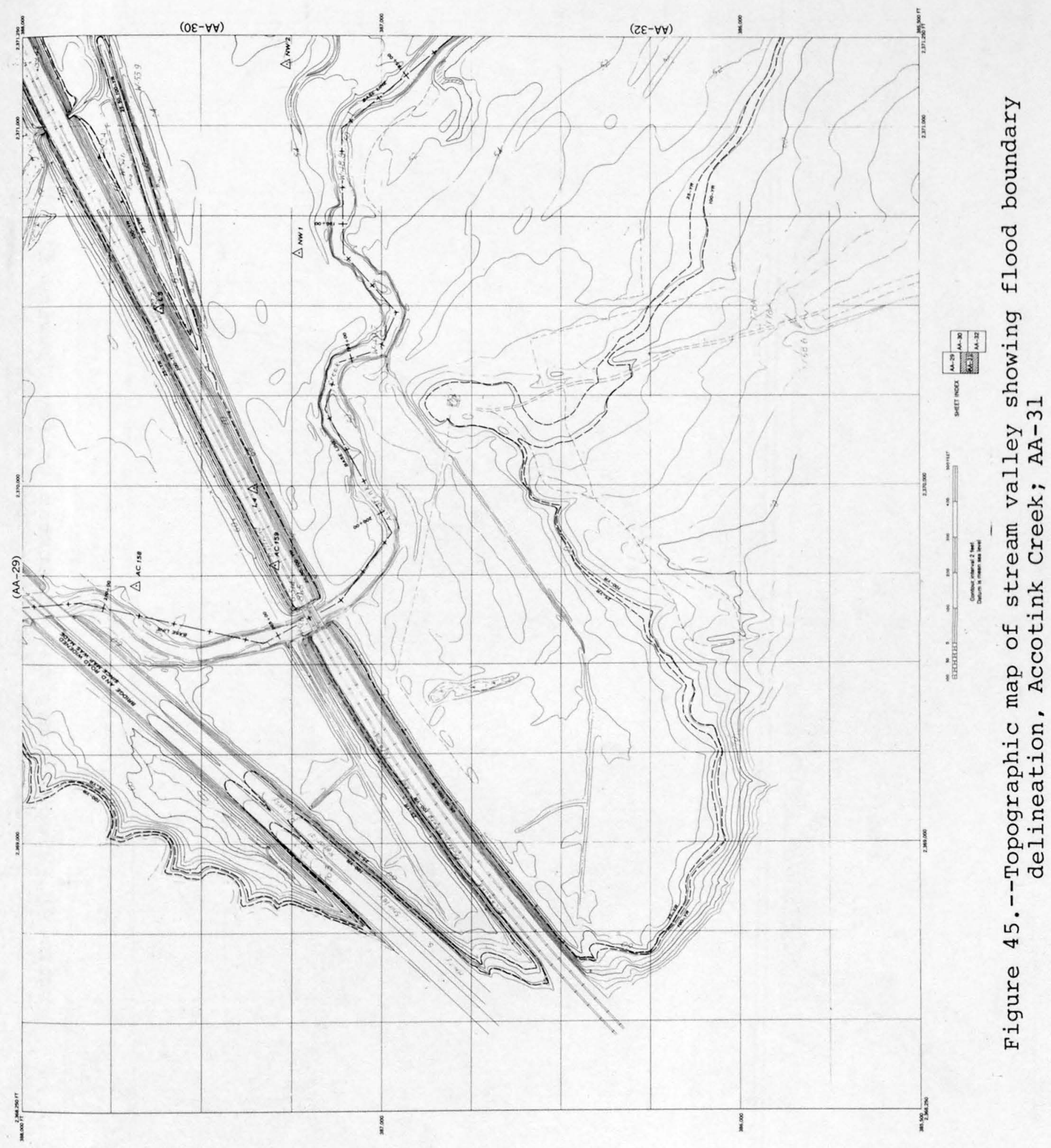

i. 89 


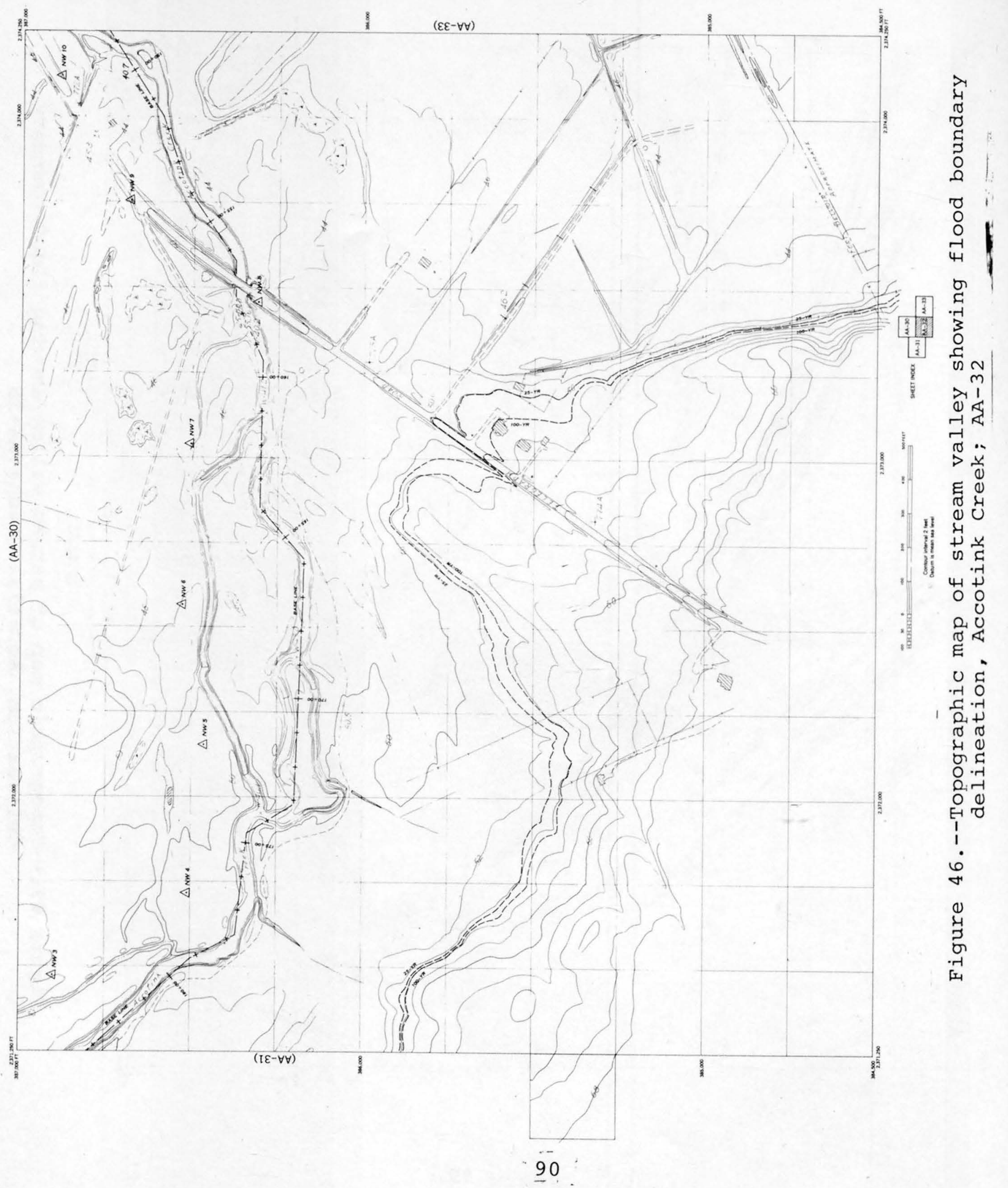




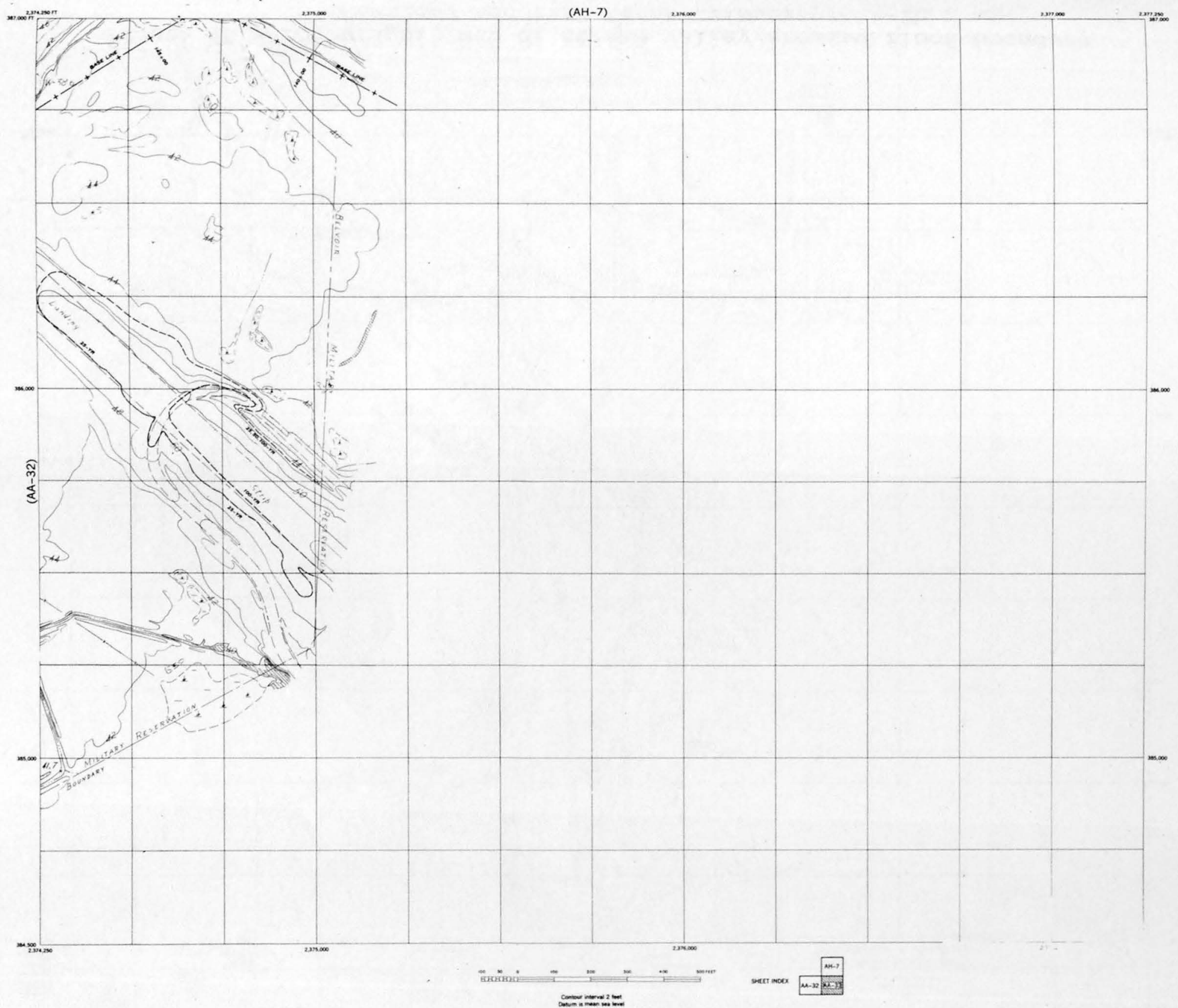

Figure 47.--Topographic map of stream valley showing flood boundary delineation, Accotink Creek; AA-33 


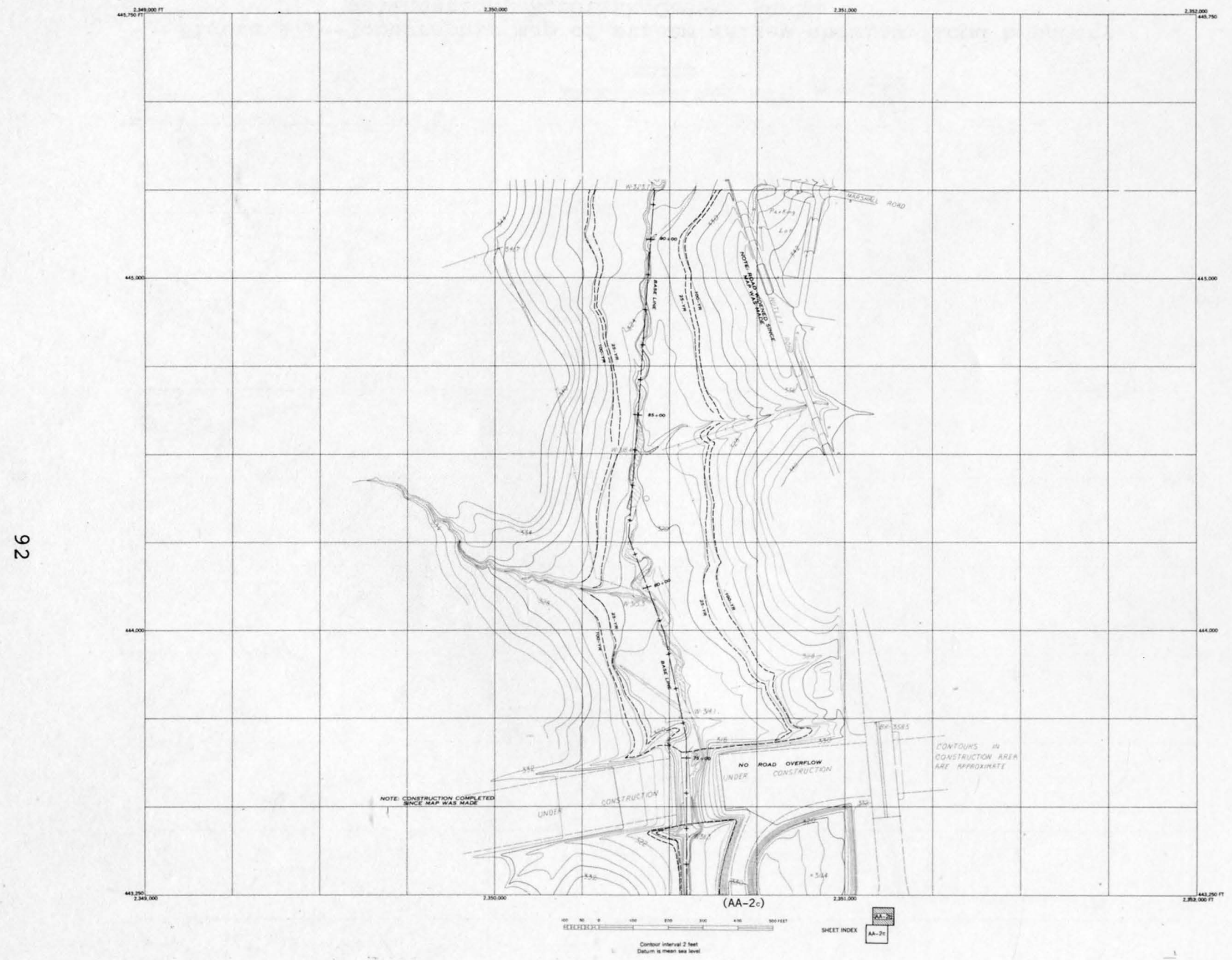

Figure 48.--Topographic map of stream valley showing flood boundary delineation, Accotink Creek Tributary; $\mathrm{AA}-2 \mathrm{~b}$ 


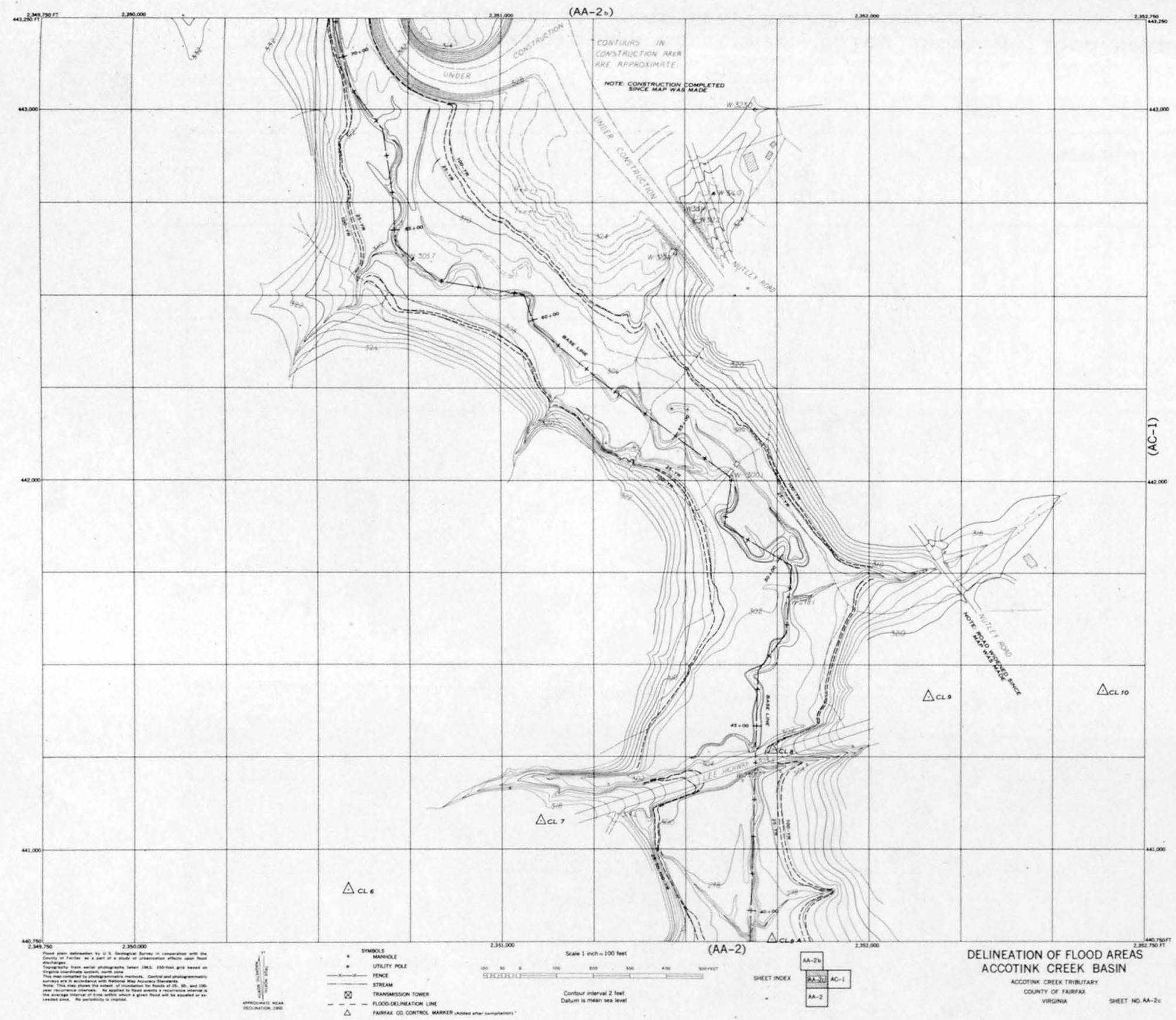

Figure 49.--Topographic map of stream valley showing flood boundary delineation, Accotink Creek Tributary; AA-2C 


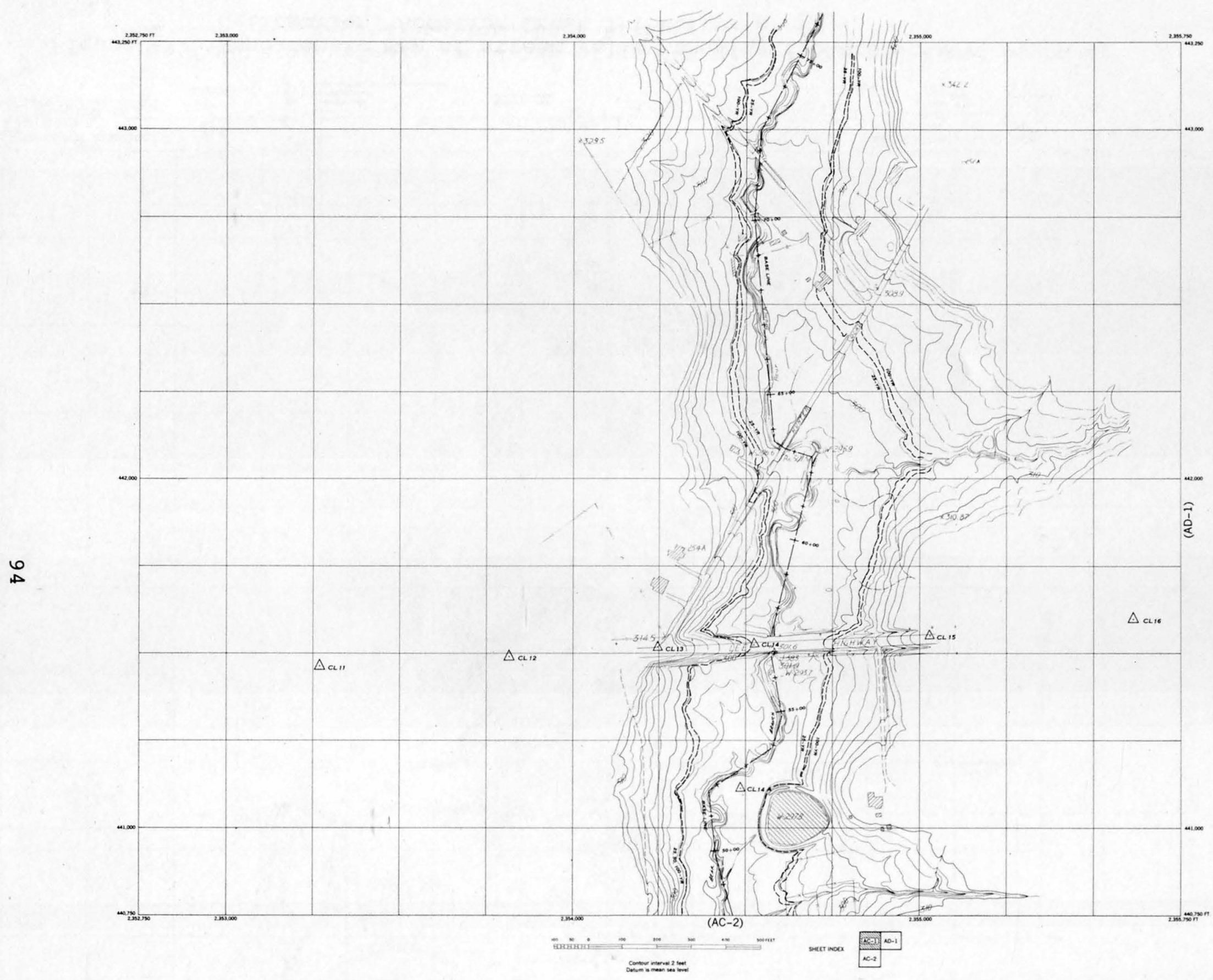

Figure 50.--Topographic map of stream valley showing flood boundary delineation, Bear Branch; AC-1 


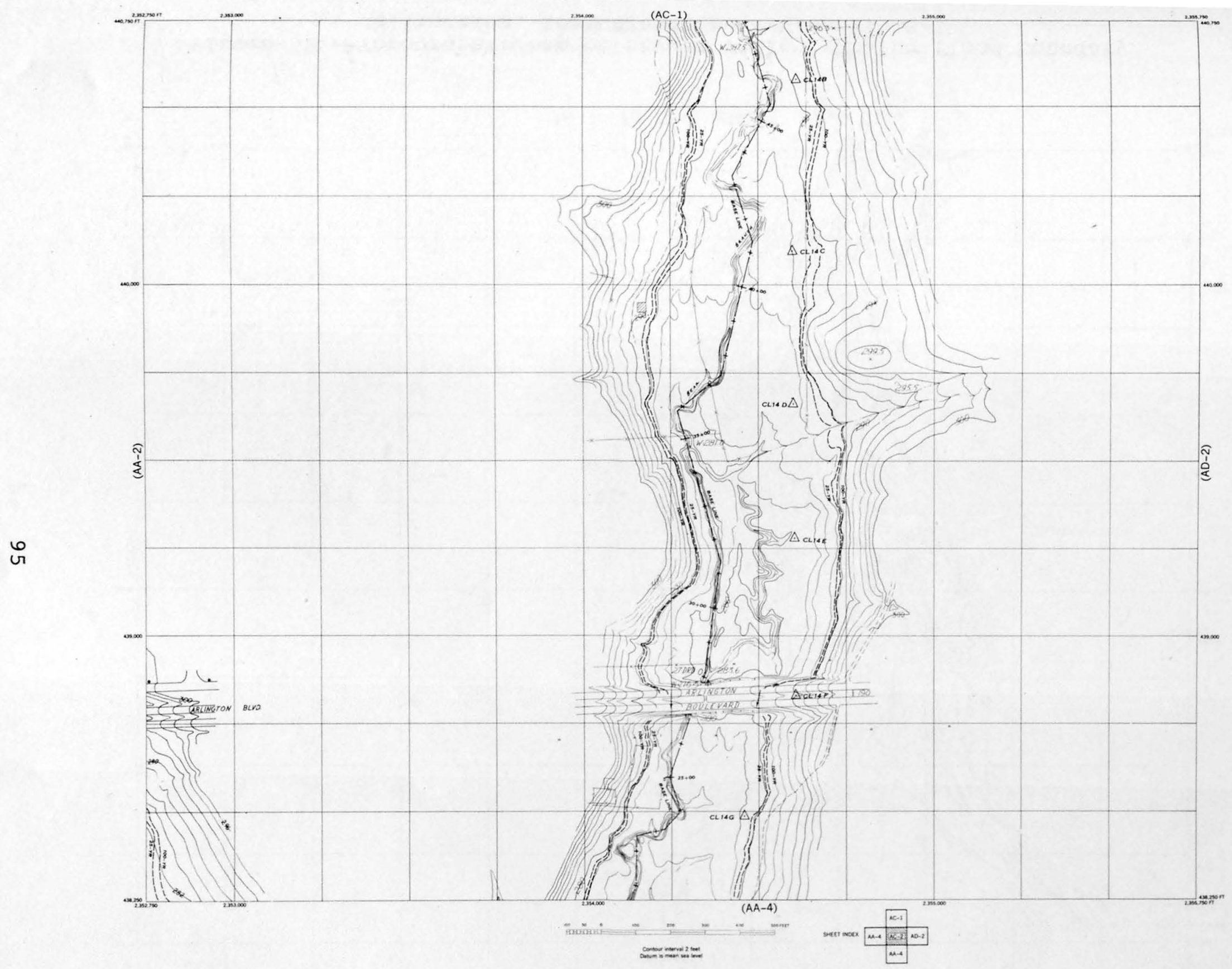

Figure 51.--Topographic map of stream valley showing flood boundary delineation, Bear Branch; AC-2 


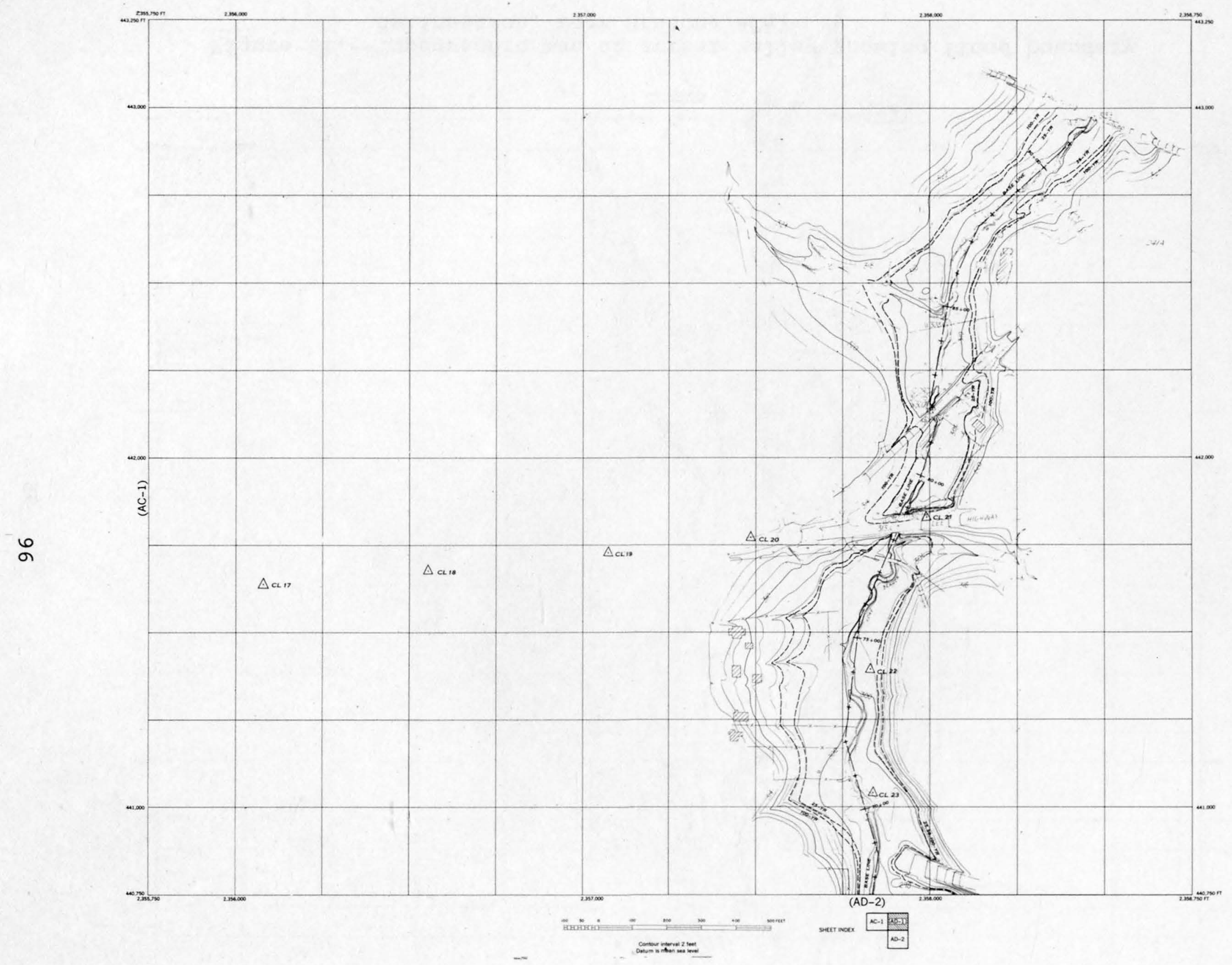

Figure 52.--Topographic map of stream valley showing flood boundary delineation, Long Branch near Vienna; $\mathrm{AD}-1$ 


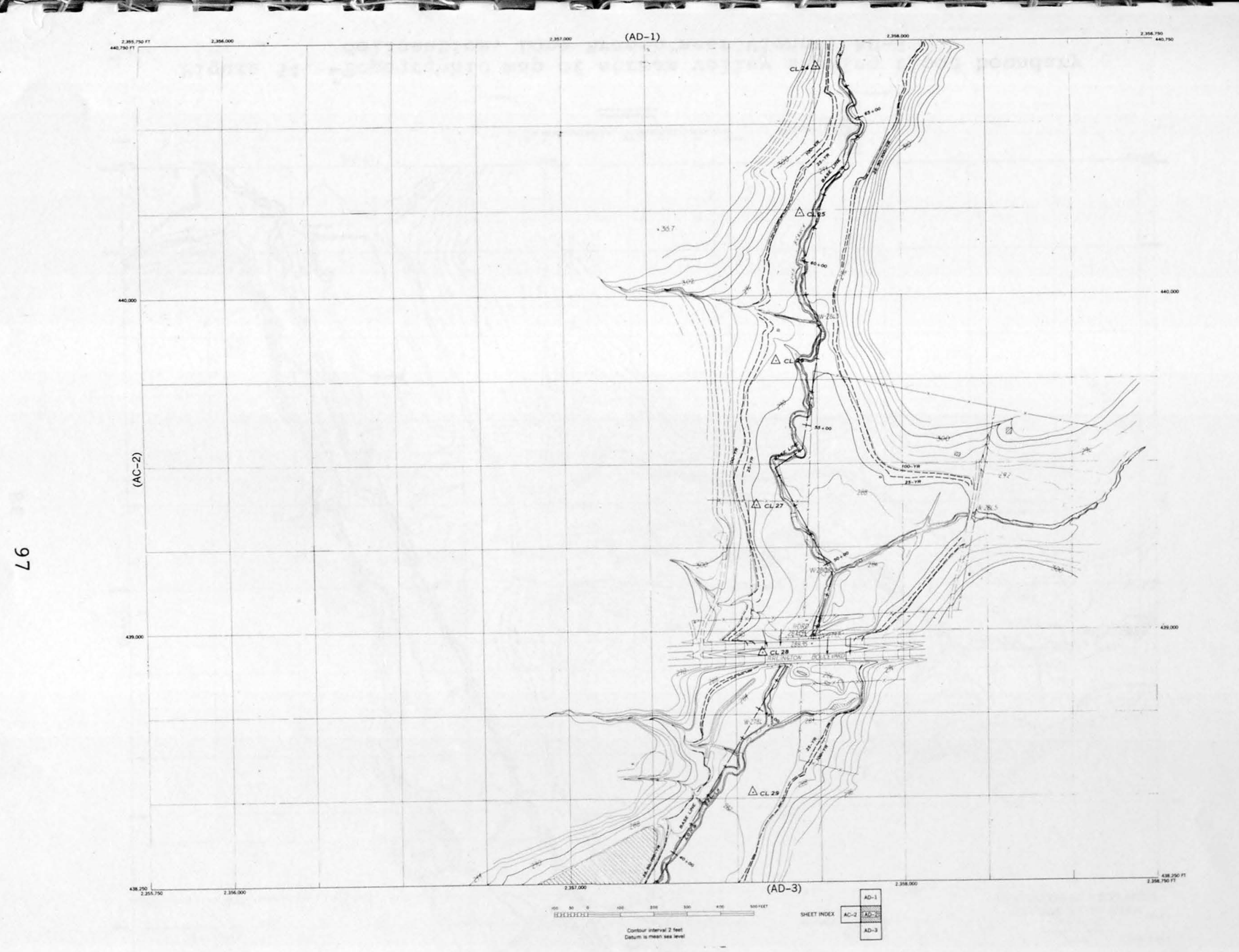

Figure 53.--Topographic map of stream valley showing flood boundary delineation, Lonq Branch near Vienna; $A D-2$ 


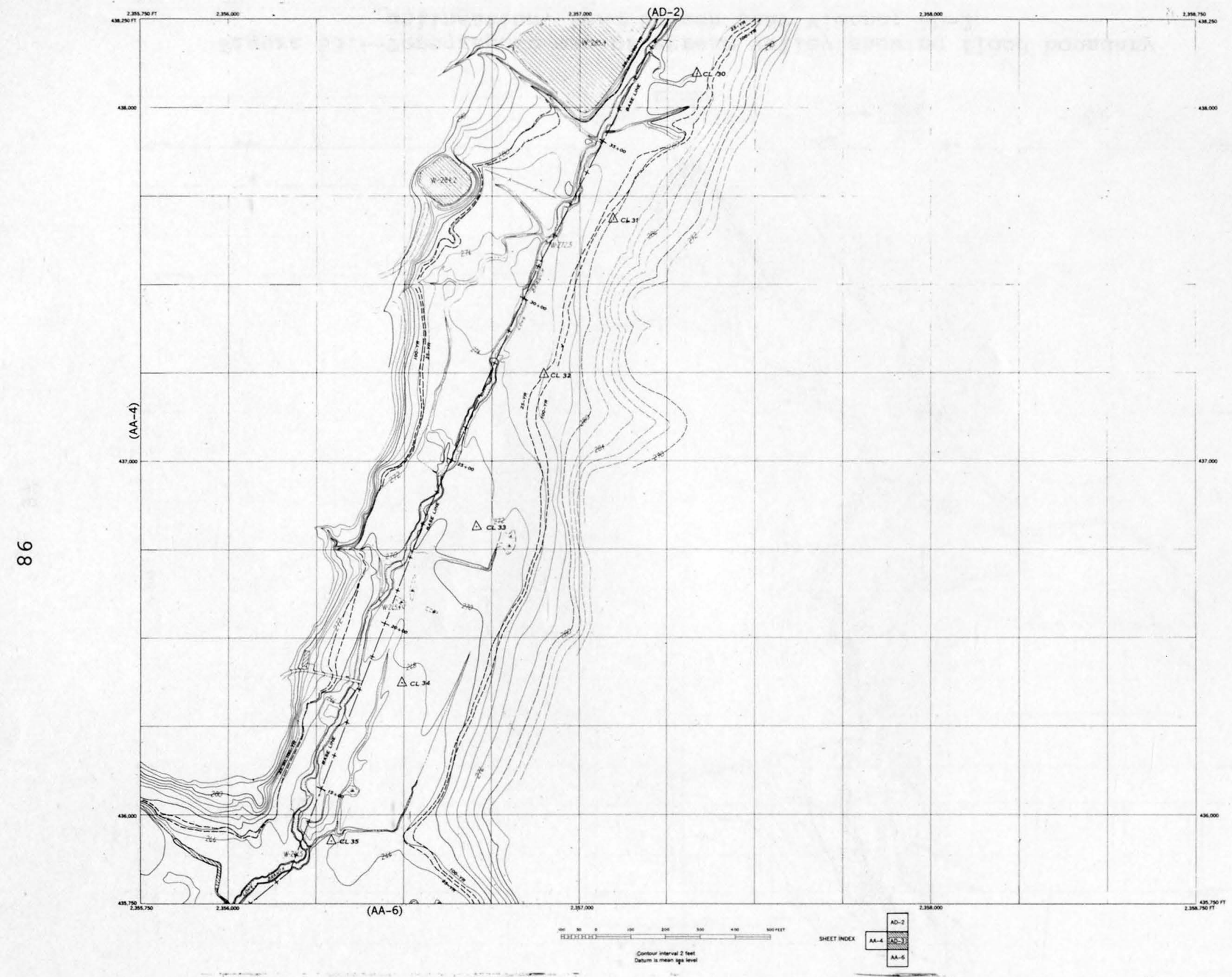

Figure 54.--Topographic map of stream valley showing flood boundary delineation, Long Branch near Vienna; $\mathrm{AD}-3$ 


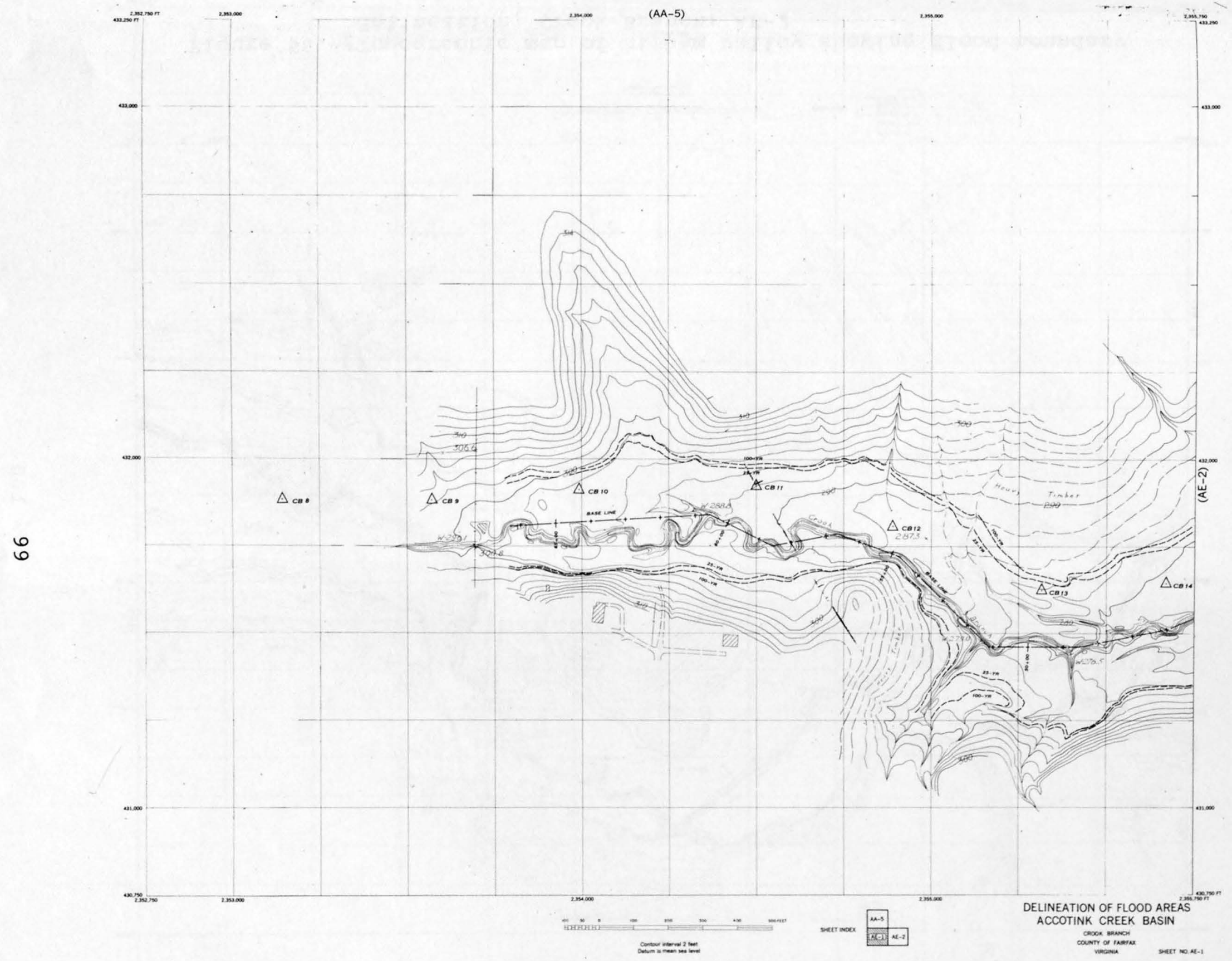

Figure 55.--Topographic map of stream valley showing flood boundary delineation, Crook Branch; AE-1 


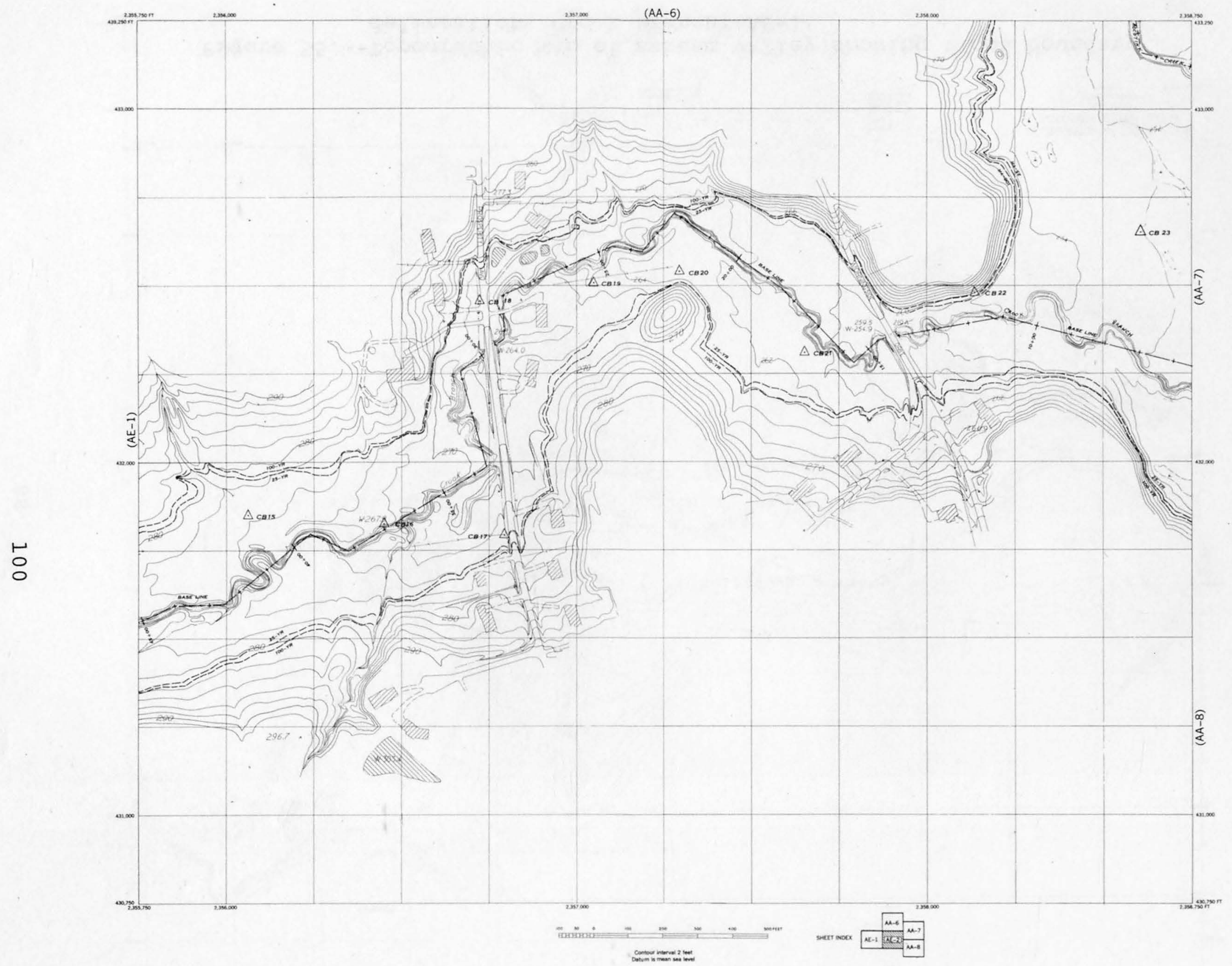

Figure 56.--Topographic map of stream valley showing flood boundary delineation, Crook Branch; $\mathrm{AE}-2$ 


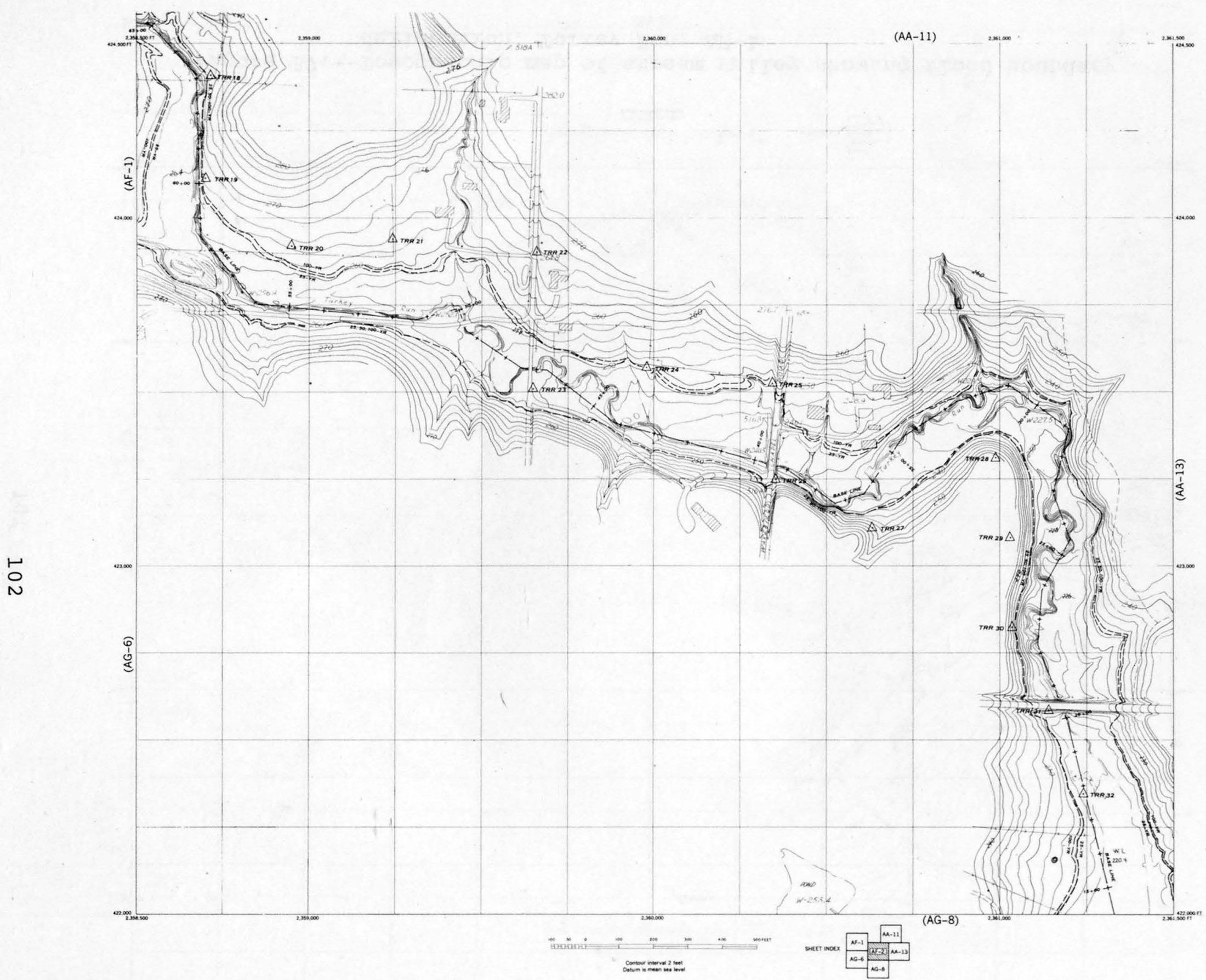

Figure 58.--Topographic map of stream valley showing flood boundary delineation, Turkey Run; AF-2 


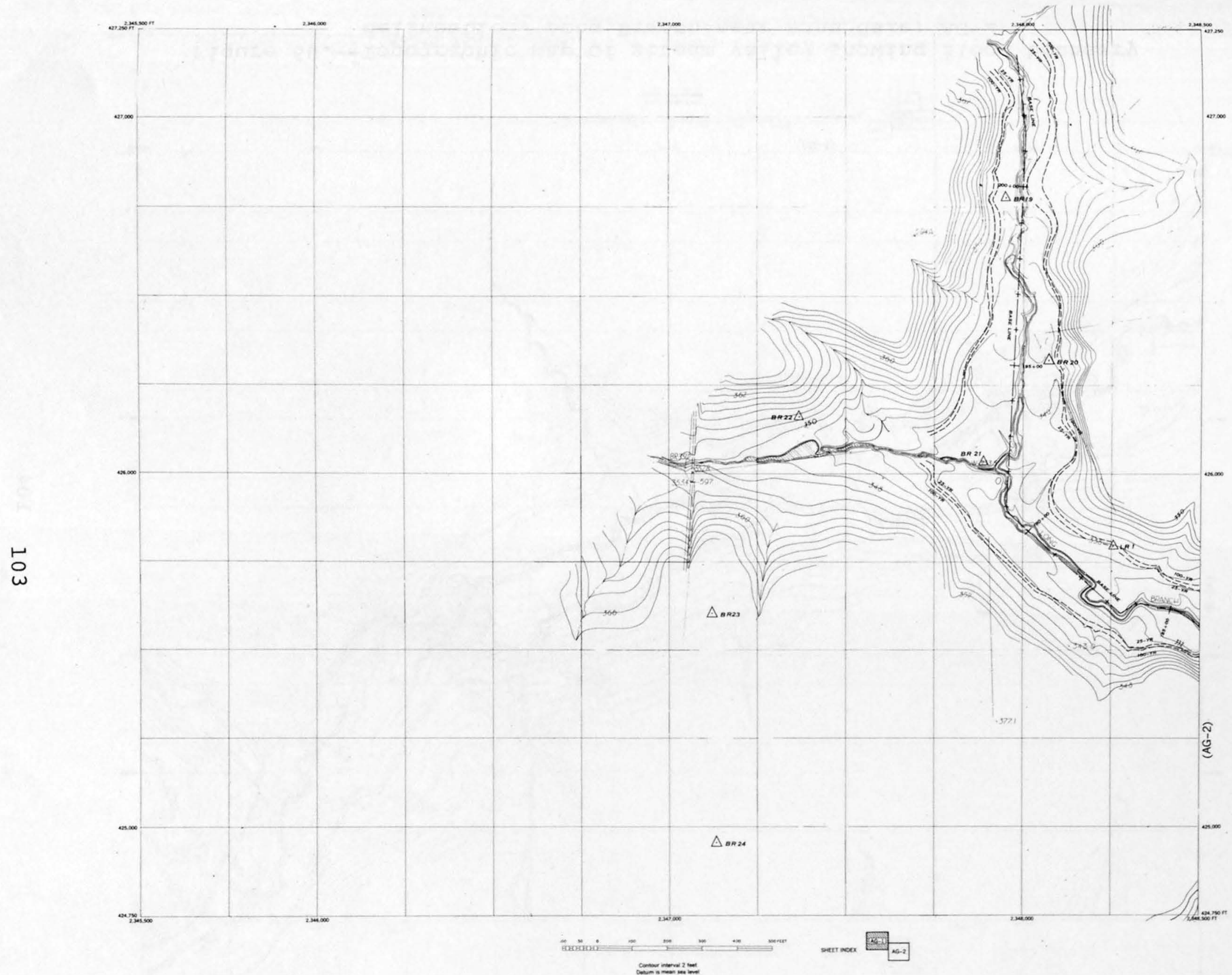

Figure 59.--Topographic map of stream valley showing flood boundary delineation, Long Branch near Annandale; AG-1 


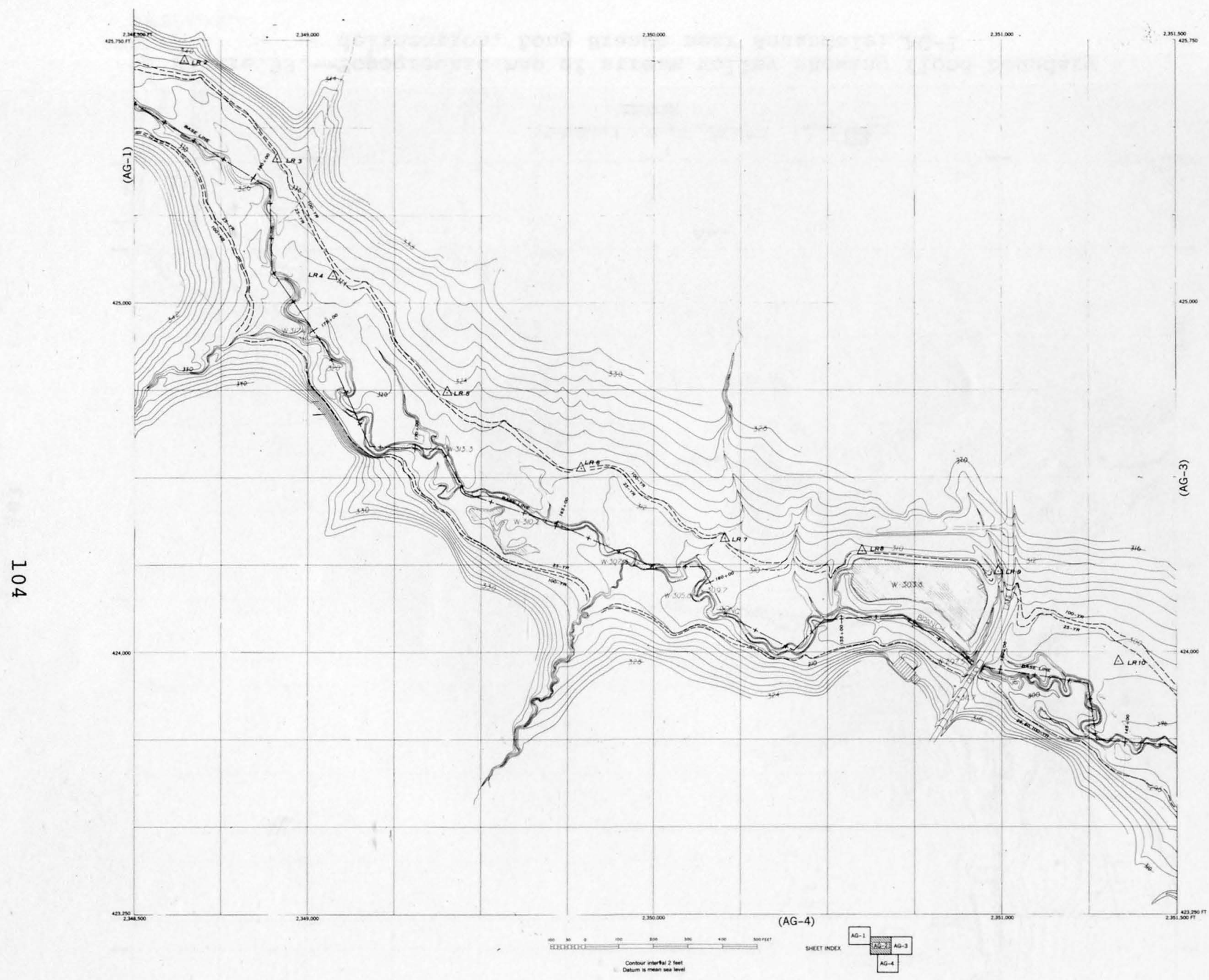

Figure 60.--Topographic map of stream valley showing flood boundary delineation, Long Branch near Annandale; AG-2 


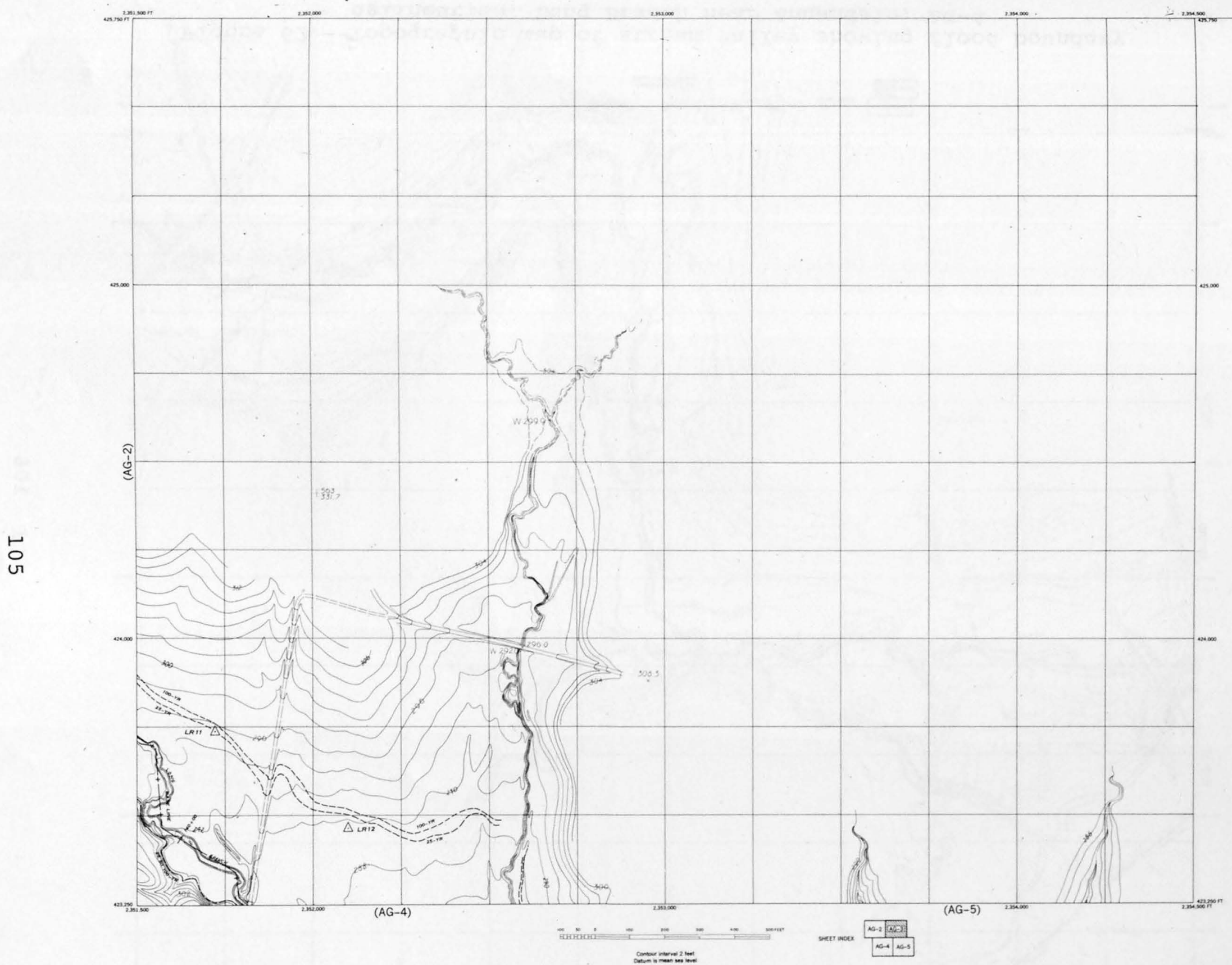

Figure 61.--Topographic map of stream valley showing flood boundary delineation, Long Branch near Annandale; AG-3 


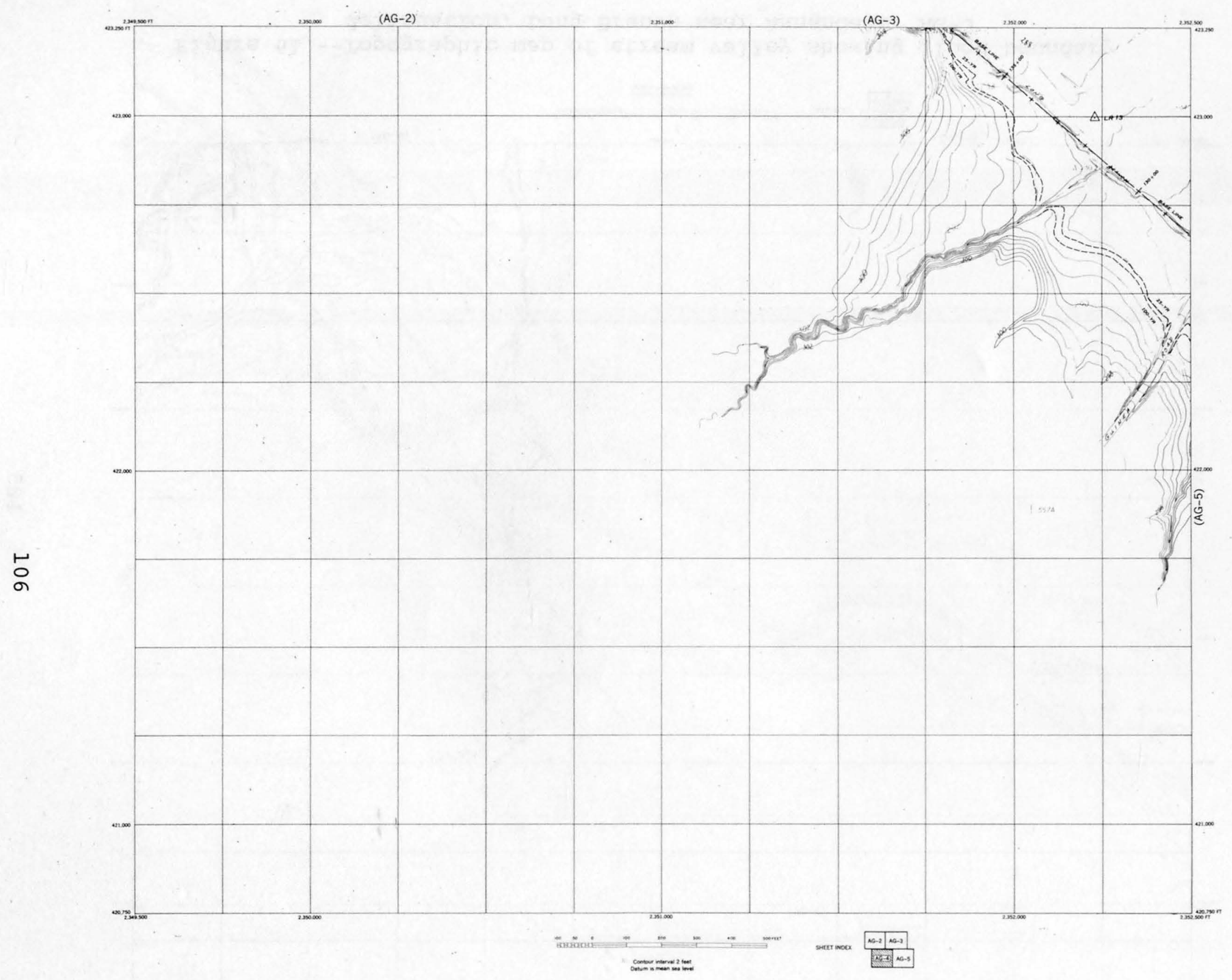

Figure 62.--Topographic map of stream valley showing flood boundary delineation, Long Branch near Annandale; AG-4 


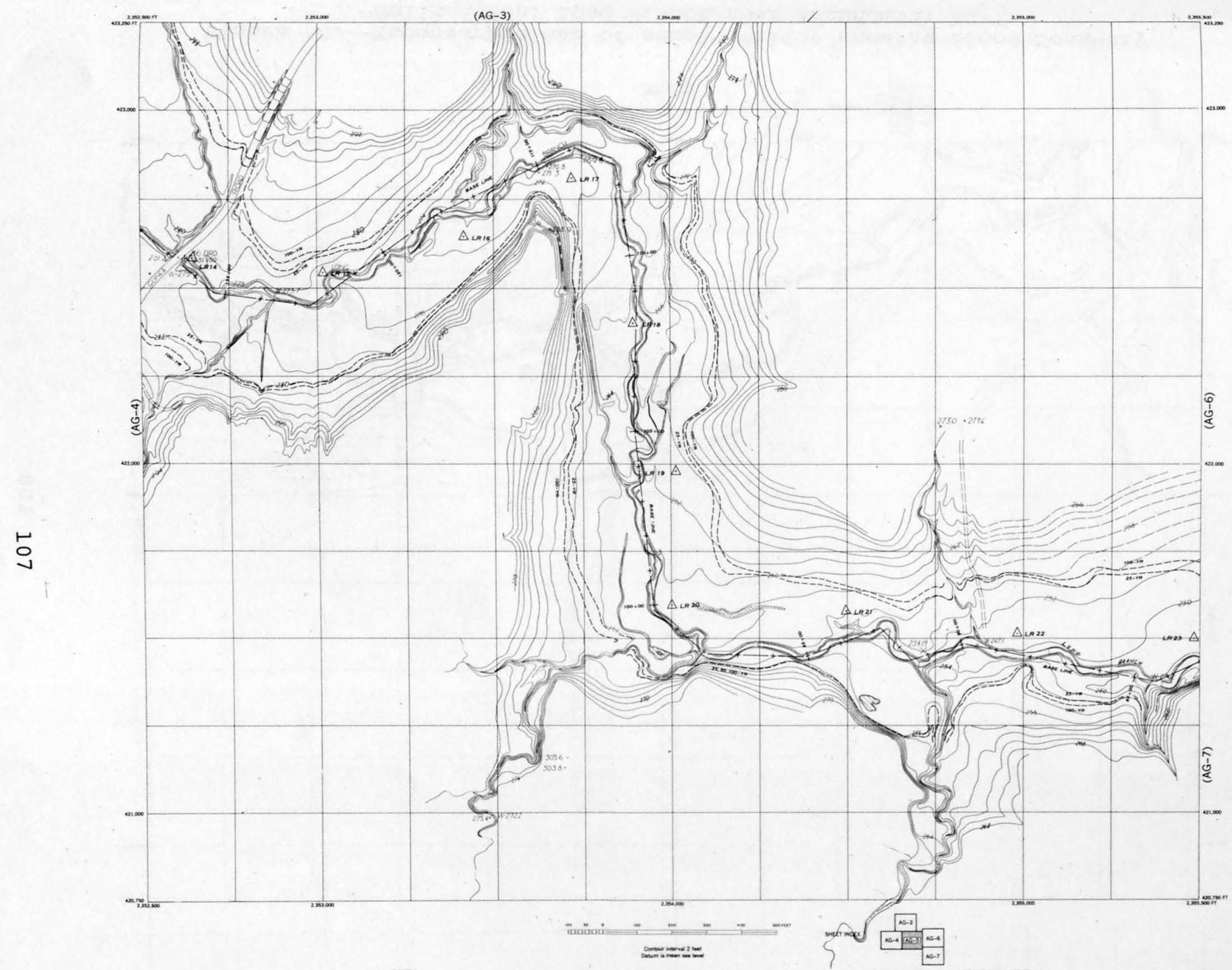

Figure 63.--Topographic map of stream valley showing flood boundary delineation, Long Branch near Annandale; AG-5 


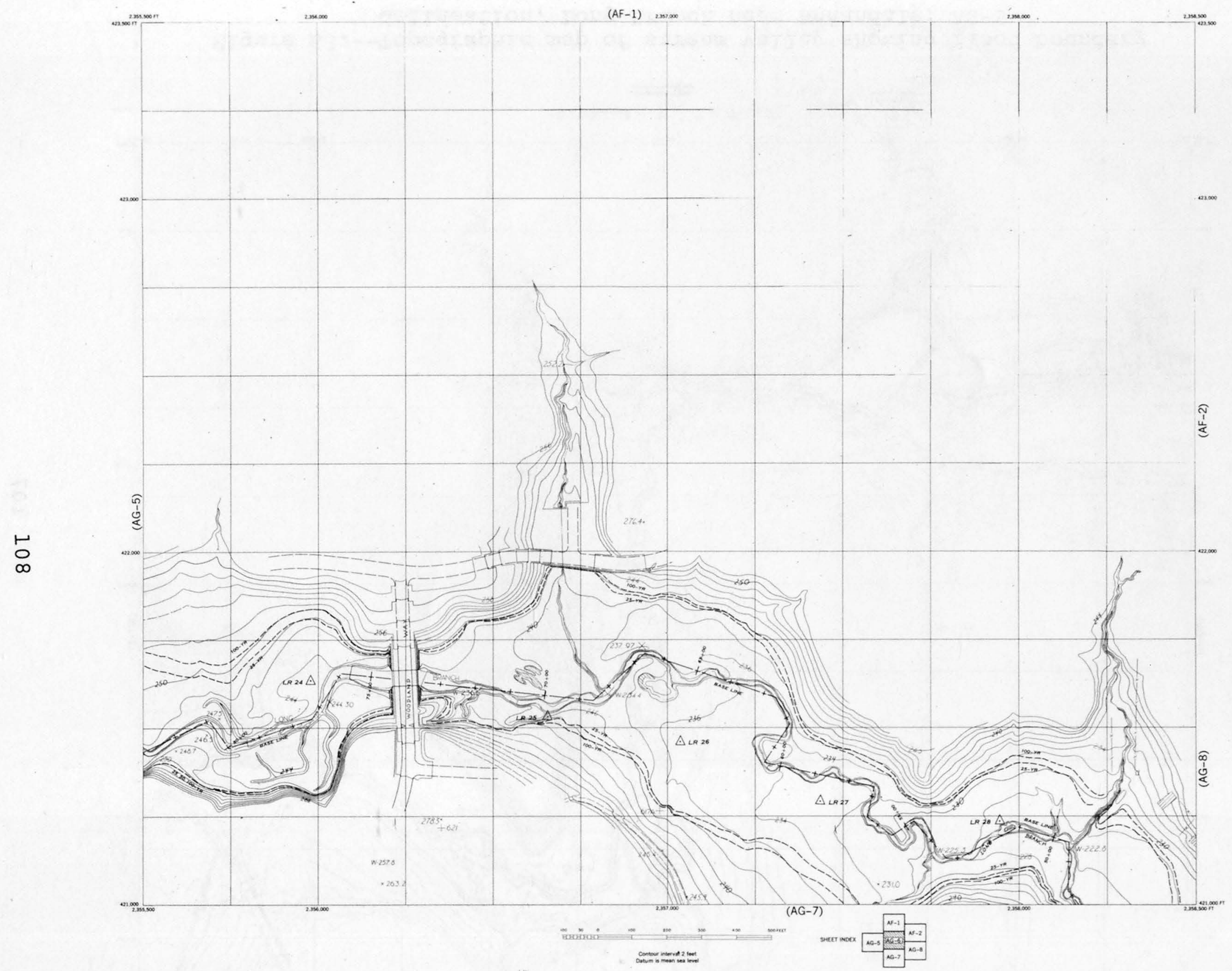

Figure 64.--Topographic map of stream valley showing flood boundary delineation, Long Branch near Annandale; AG-6 


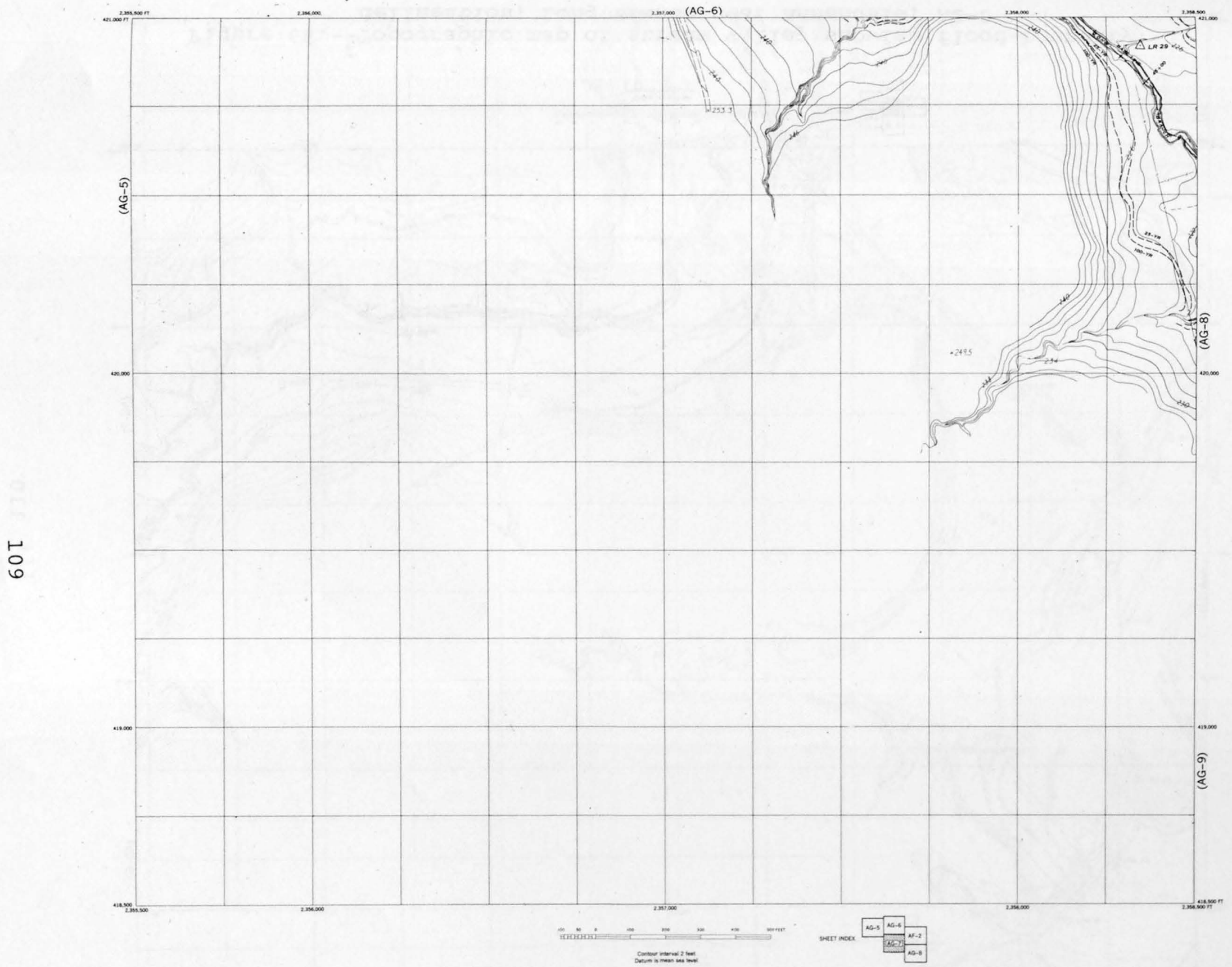

Figure 65.--Topographic map of stream valley showing flood boundary delineation, Long Branch near Annandale; AG-7 


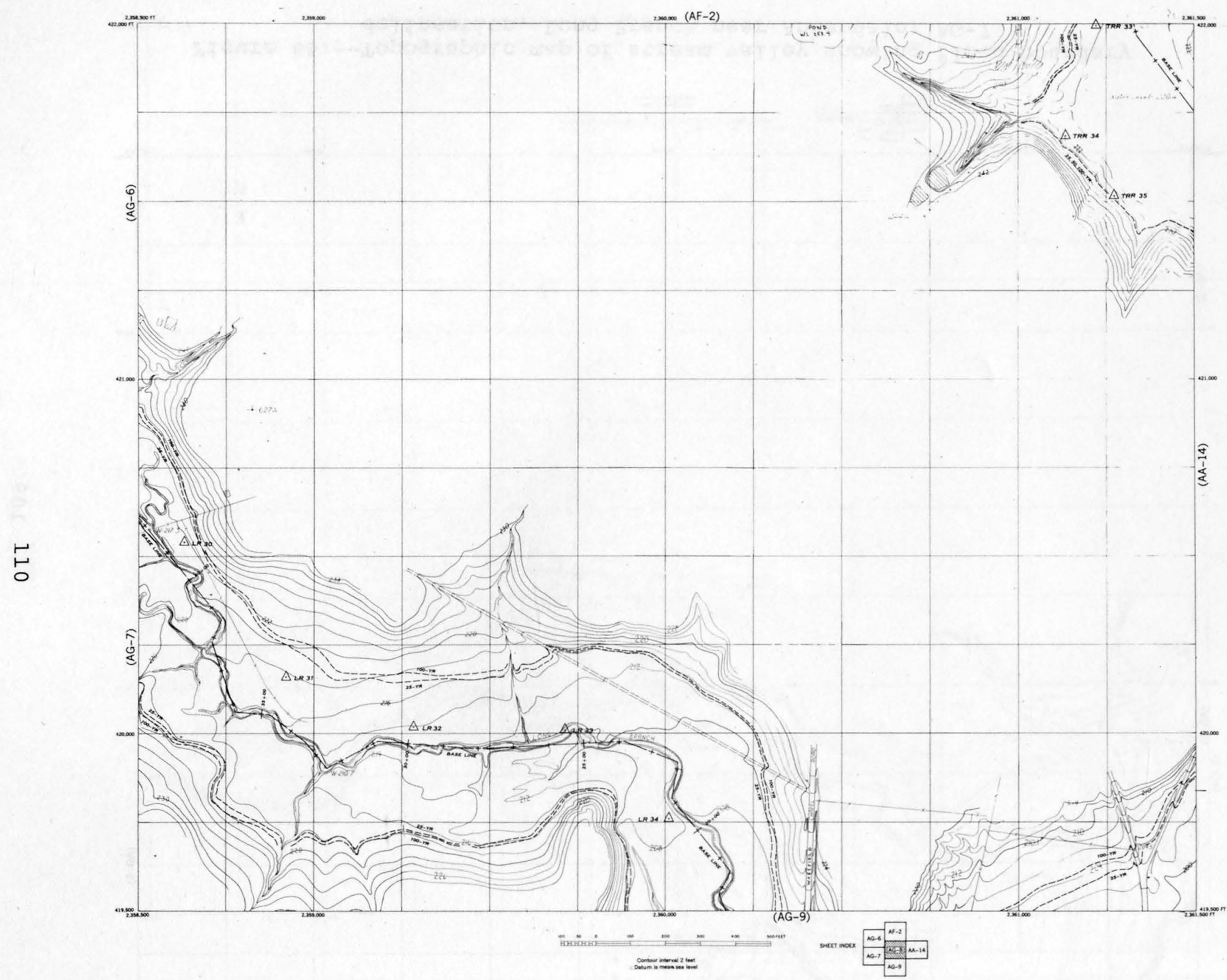

Figure 66.--Topographic map of stream valley showing flood boundary delineation, Long Branch near Annandale; AG-8 


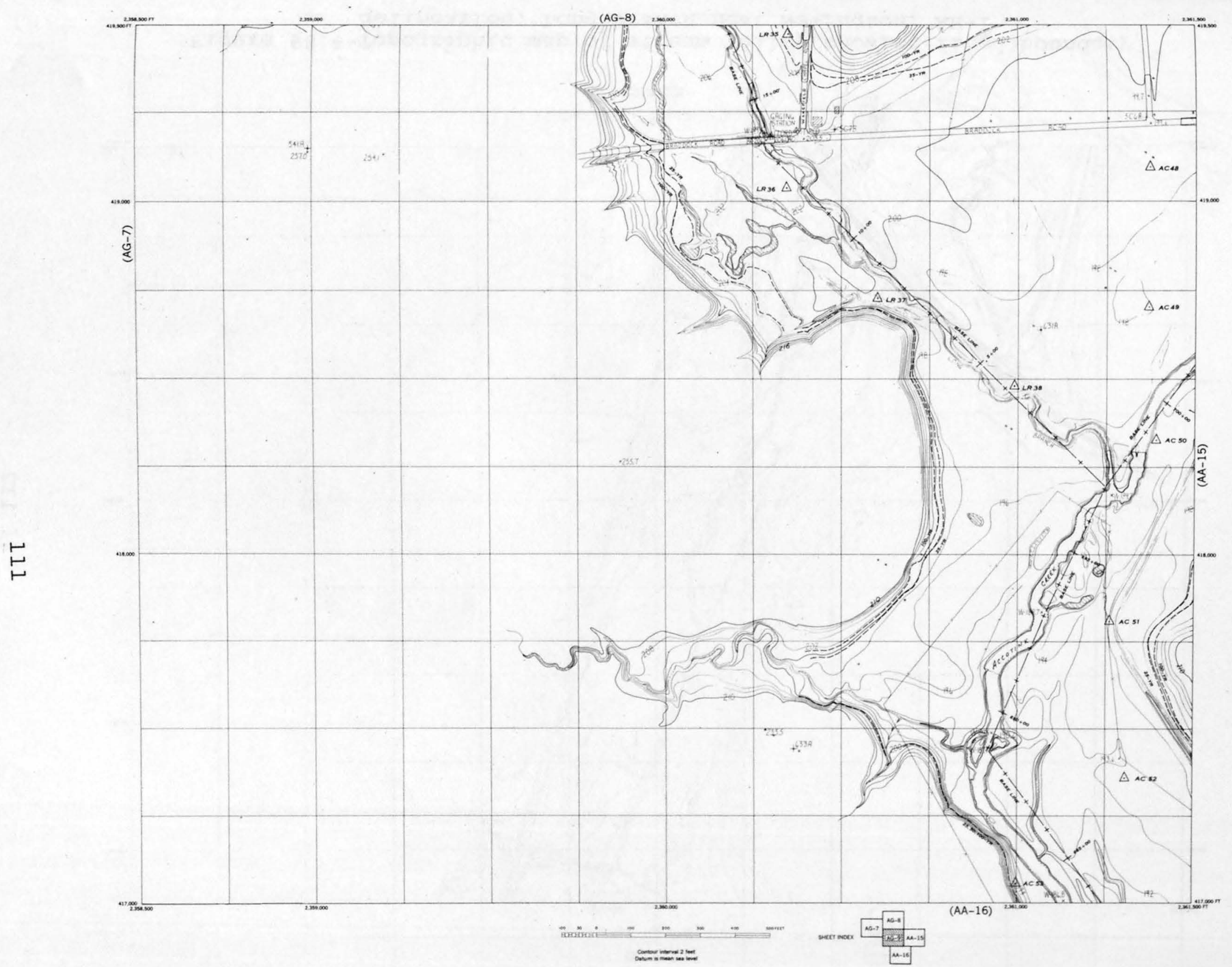

Figure 67.--Topographiq map of stream valley showing flood boundary delineation, Long Branch near Annandale; AG-9 


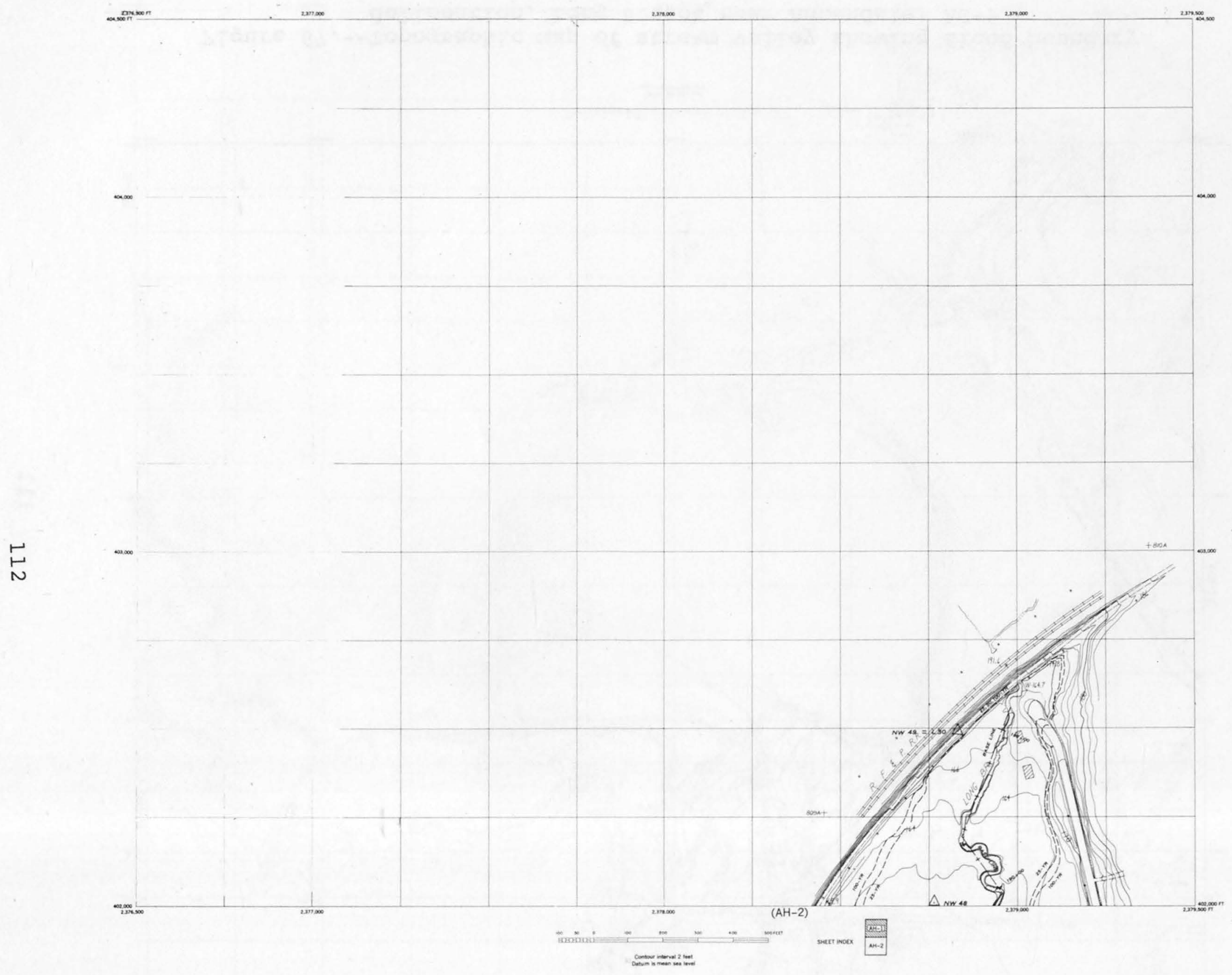

Figure 68.--Topographic map of stream valley showing flood boundary delineation, Long Branch near Newington; $\mathrm{AH}-1$ 


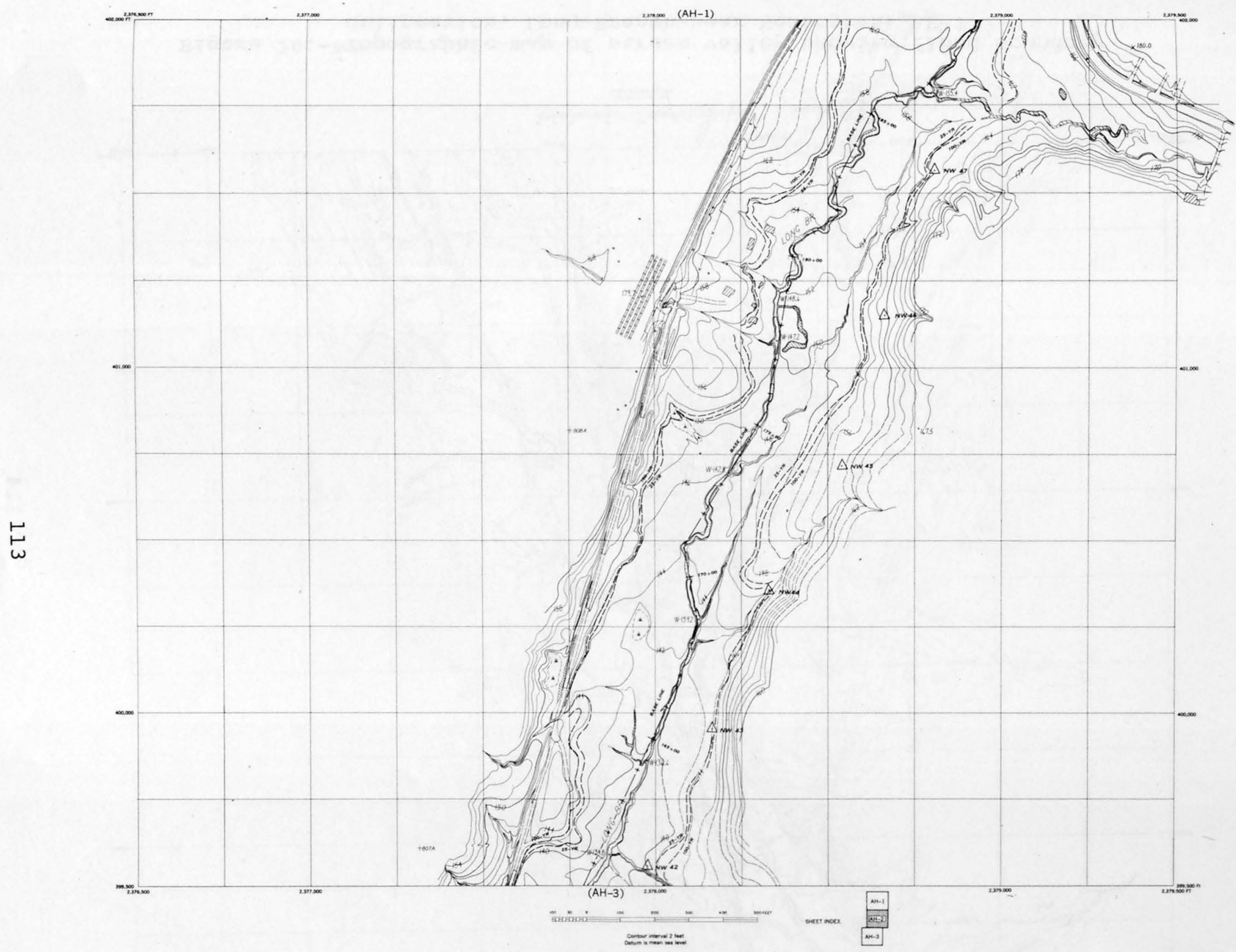

Figure 69.--Topographic map of stream valley showing flood boundary delineation, Long Branch near Newington; AH-2 


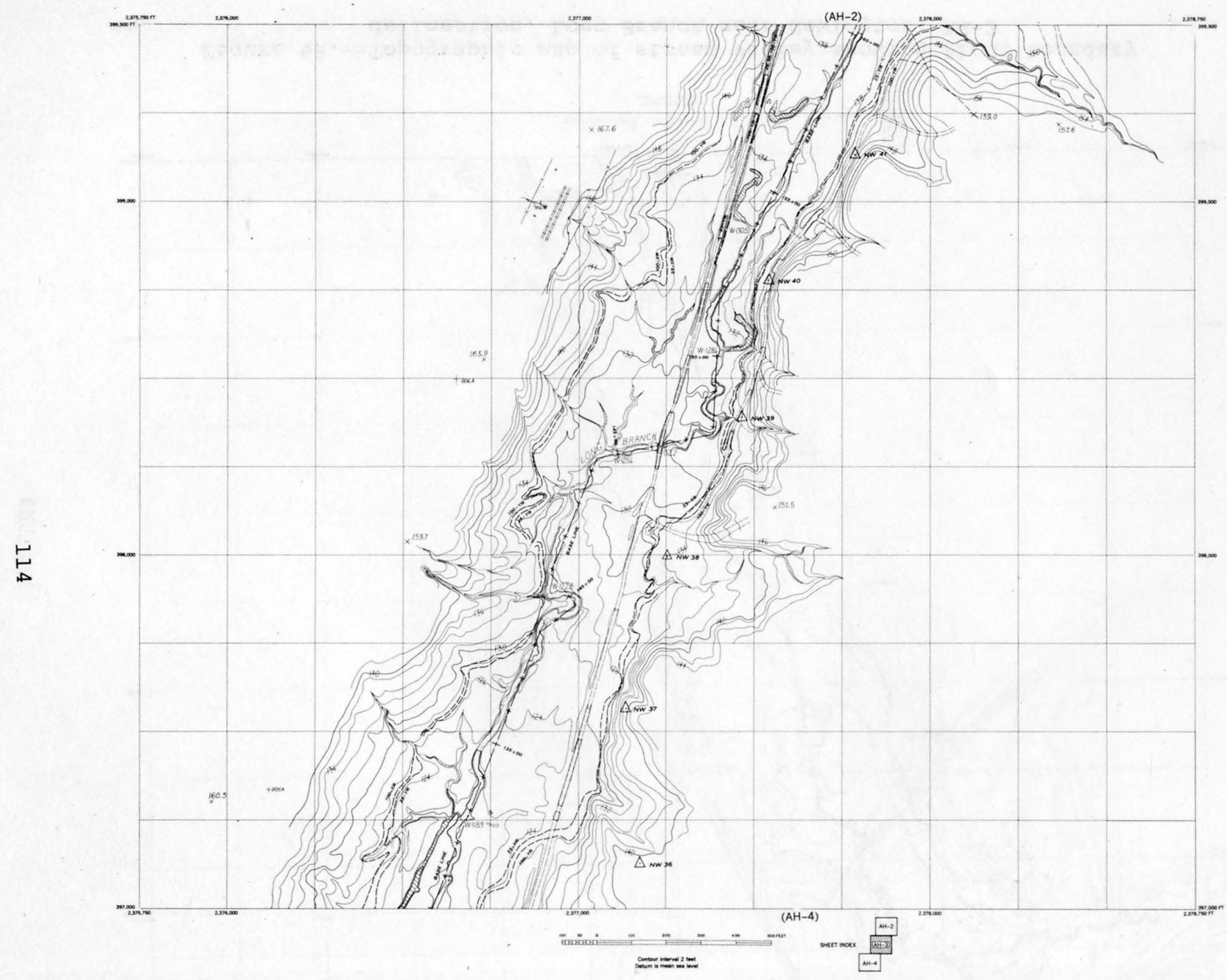

Figure 70.--Topographic map of stream valley showing flood boundary delineation, Long Branch near Newington; $\mathrm{AH}-3$ 


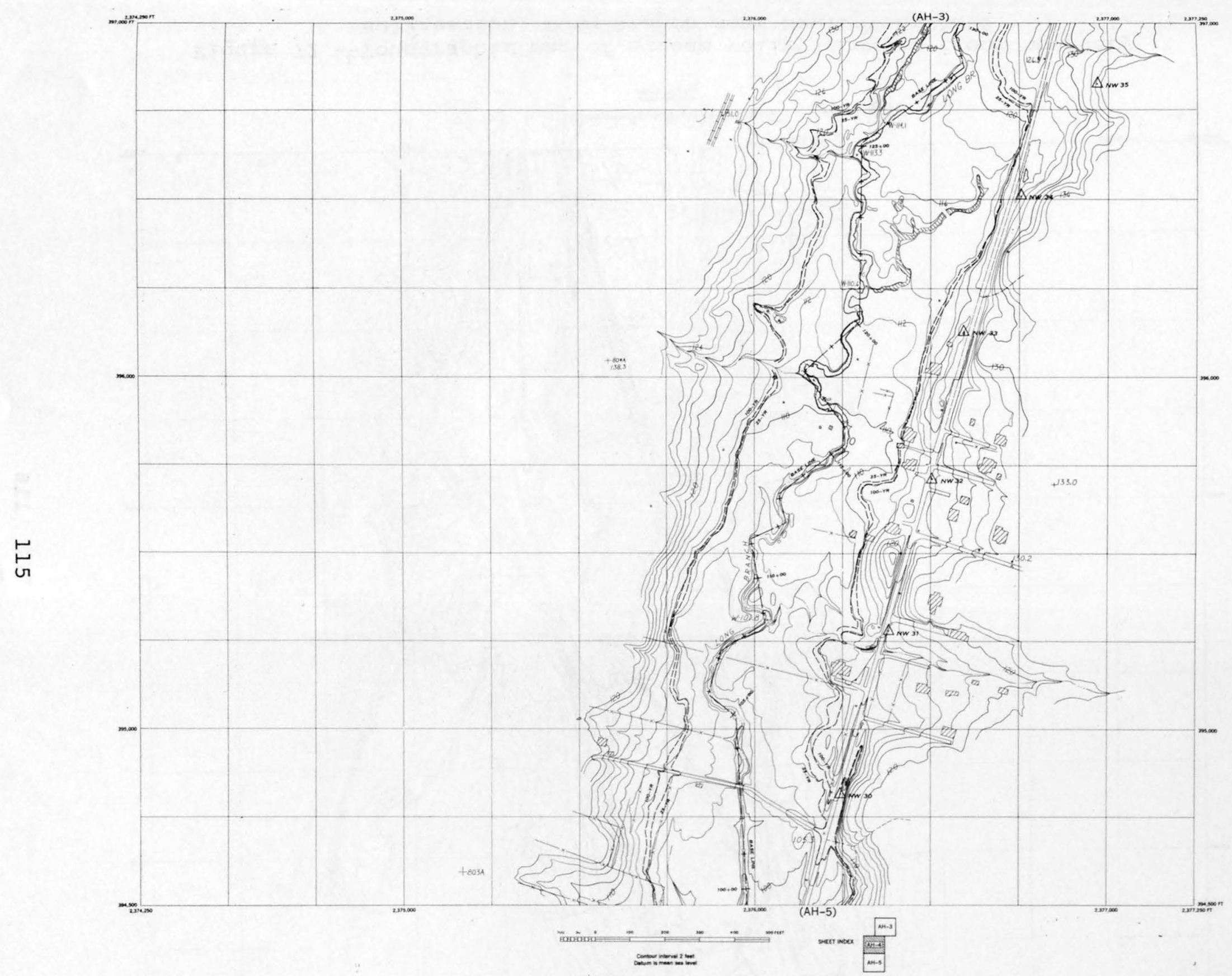

Figure 71.--Topographic map of stream valley showing flood boundary delineation, Long Branch near Newington; $\mathrm{AH}-4$ 


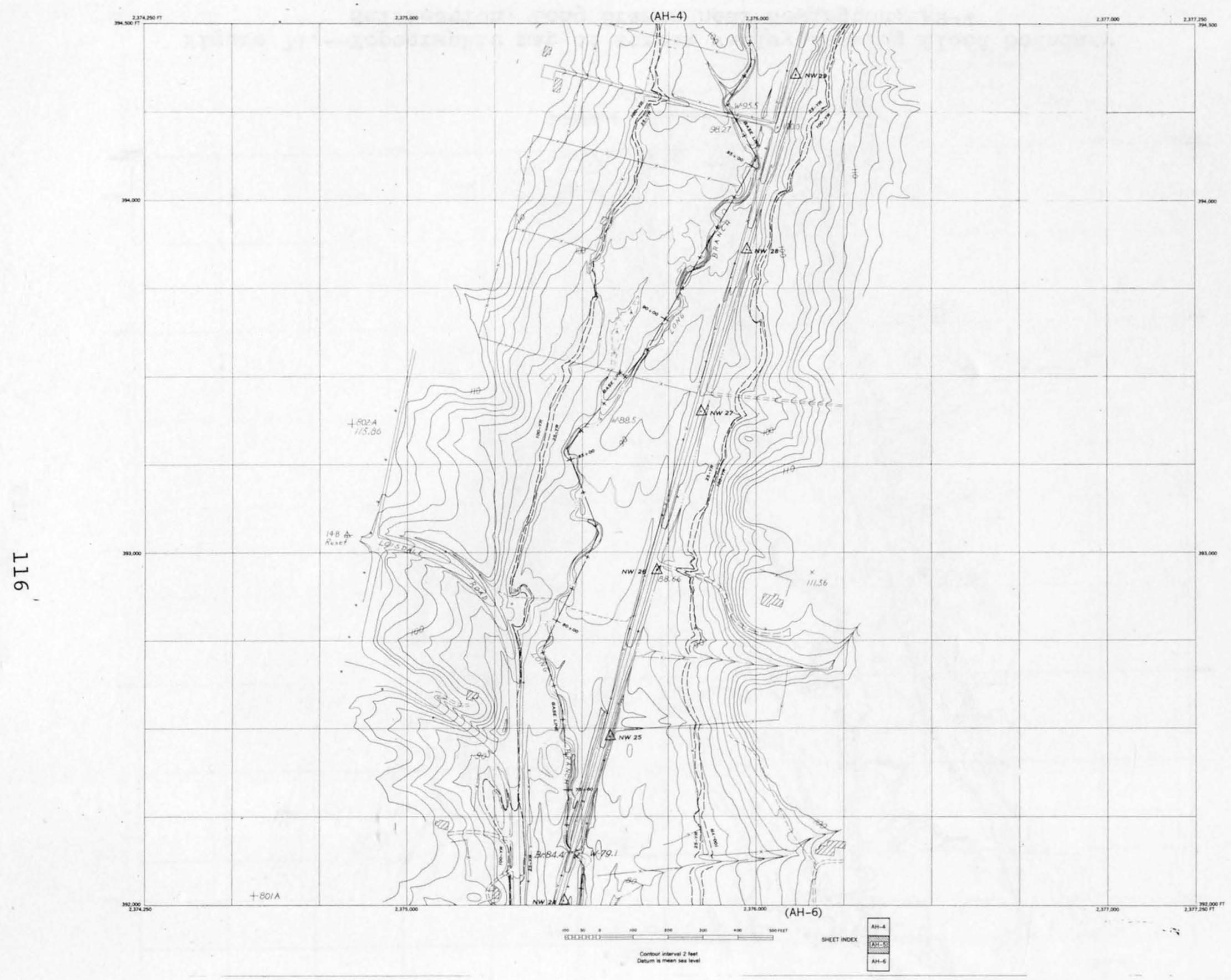

Figure 72.--Topographic map of stream valley showing flood boundary delineation, Long Branch near Newington; $\mathrm{AH}-5$ 


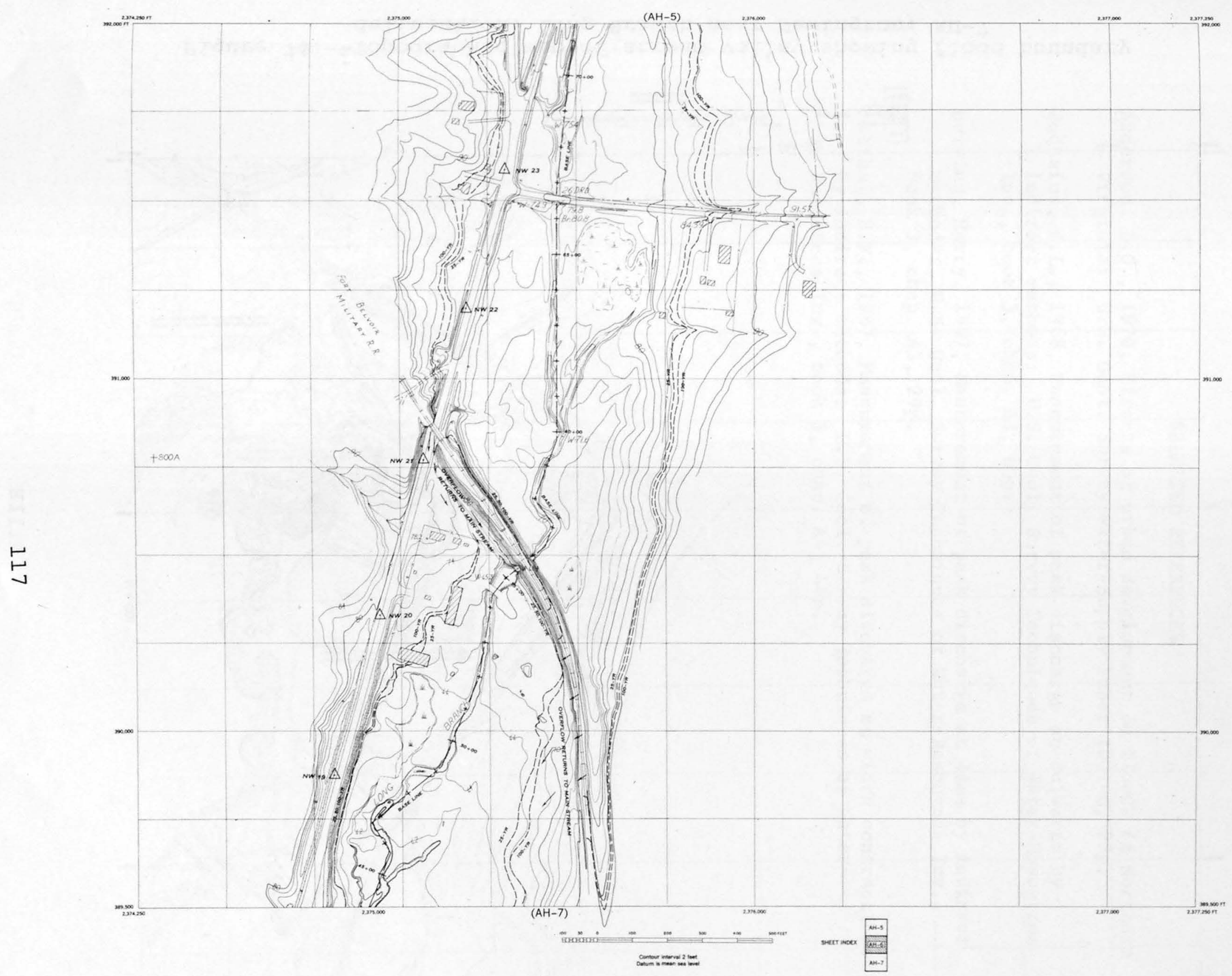

Figure 73.--Topographic map of stream valley showing flood boundary delineation, Long Branch near Newington; $\mathrm{AH}-6$ 


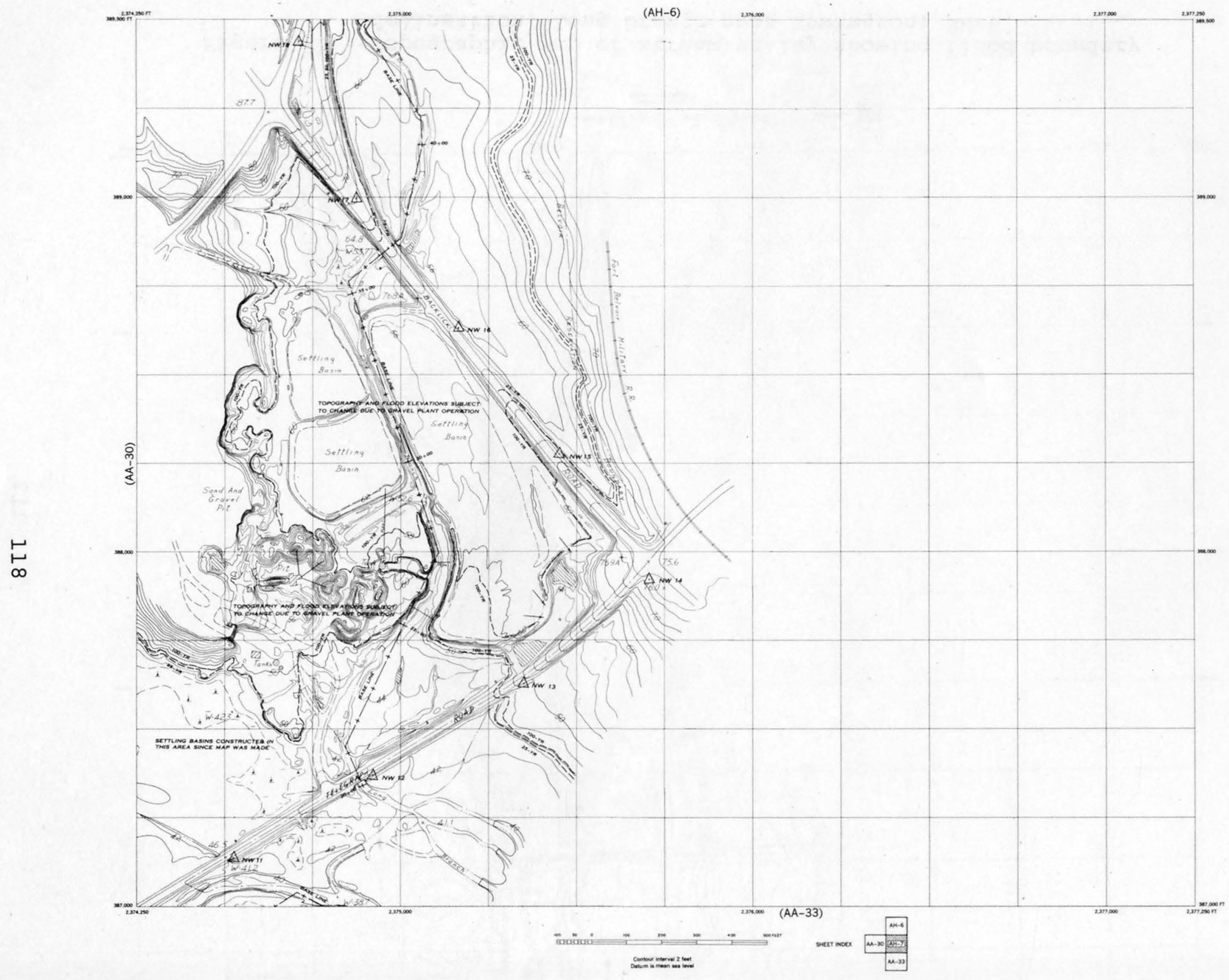

Figure 74.--Topographic map of stream valley showing flood boundary delineation, Long Branch near Newington; $\mathrm{AH}-7$ 


\section{SELECTED REFERENCES}

Anderson, D.G., 1970, Effects of urban development on floods in Northern Virginia: U.S. Geo1. Survey Water-Supply Paper 2001-C, 22p.

Bodhaine, G.L., 1968, Measurement of peak discharge at culverts by indirect methods: U.S. Geol. Survey Techniques of Water-Resources Inv., book 3, chap. A3, 60p.

Hulsing, Harry, 1967, Measurement of peak discharge at dams by indirect methods: U.S. Geo1. Survey Techniques of Water-Resources Inv., book 3, chap. A5, 29p.

Matthai, H.F., 1967, Measurement of peak discharge at width contractions by indirect methods: U.S. Geol. Survey Techniques of WaterResources Inv., book 3, chap. A4, 44p. 
